JOURNAL OF THE

AMERICAN MATHEMATICAL SOCIETY

Volume 24, Number 4, October 2011, Pages 1051-1103

S 0894-0347(2011)00700-5

Article electronically published on April 6, 2011

\title{
MASS EQUIDISTRIBUTION FOR AUTOMORPHIC FORMS OF COHOMOLOGICAL TYPE ON $G L_{2}$
}

\author{
SIMON MARSHALL
}

\section{INTRODUCTION}

One of the central problems in the subject of quantum chaos is to understand the behaviour of high energy Laplace eigenfunctions on a Riemannian manifold $M$. There is an important conjecture of Rudnick and Sarnak 32] which predicts one aspect of this behaviour in the case when $M$ is compact and negatively curved, namely that the microlocal lifts of eigenfunctions tend weakly to Liouville measure on the unit tangent bundle. This is known as the quantum unique ergodicity conjecture and has as a corollary that the $L^{2}$ mass of eigenfunctions becomes weakly equidistributed on $M$. We refer the reader to [20, 21, 32, 37, 38, 42, 43, for many illuminating discussions and interesting results related to this conjecture.

In this paper we shall deal with a variant of Rudnick and Sarnak's conjecture which replaces Laplace eigenfunctions with certain modular forms. This may be described most easily in the case of the modular surface $X=S L(2, \mathbb{Z}) \backslash \mathbb{H}^{2}$, where the objects we shall consider are holomorphic modular forms of large weight, or equivalently sections of high tensor powers of the line bundle of holomorphic differentials on $X$. If $f$ is a holomorphic modular cusp form of weight $k$, the analogue of the $L^{2}$ mass of $f$ is the Petersson measure

$$
\mu_{f}=y^{k}|f(z)|^{2} d v
$$

where $d v$ denotes the hyperbolic volume. The measure $\mu_{f}$ is invariant under $S L(2, \mathbb{Z})$, and we may suppose that $f$ has been normalised so that it descends to a probability measure on $X$. The analogue of the quantum unique ergodicity conjecture for holomorphic forms is then to show that the measures $\mu_{f}$ tend weakly to the hyperbolic volume as the weight of $f$ tends to infinity. This is very much in the spirit of the original conjectures, with the Cauchy-Riemann equations replacing the Laplace operator and the weight $k$ playing the role of the eigenvalue, and was considered in [23, 34].

There are two main differences between this conjecture and the classical form of QUE. The first is that no microlocal lift is known for holomorphic forms, so we are restricted to considering an equidistribution on $X$ rather than its unit tangent bundle, and ergodic methods may not presently be applied to this problem. The second is that the literal analogue of the conjecture fails because the space of cusp forms is large and contains elements such as $\Delta^{k}$ (where $\Delta$ is Ramanujan's cusp form) whose mass is not equidistributing. From a number-theoretic point of view it is

Received by the editors July 1, 2010 and, in revised form, December 10, 2010, and February $22,2011$.

2010 Mathematics Subject Classification. Primary 11F41, 11F11; Secondary 11F75.

(C)2011 American Mathematical Society 1051

Reverts to public domain 28 years from publication 
natural to deal with this multiplicity issue by requiring $f$ to be a Hecke eigenform, which gives a refinement of the conjecture known as arithmetic QUE. This is a natural condition to impose, as Watson's triple product formula 41] illustrates that the generalised Riemann hypothesis would imply QUE for holomorphic Hecke eigenforms with the optimal rate of equidistribution. The first unconditional results on this conjecture were obtained by Sarnak [34, who showed that it was true for dihedral forms, and by Luo and Sarnak [23, who showed that it was true for almost all eigenforms of weight at most $k$.

In [15, 16, 39, Holowinsky and Soundararajan established QUE for all holomorphic Hecke eigenforms on the modular surface $X$, or more generally any noncompact congruence hyperbolic surface. Their proof is a combination of two different approaches, one based on bounding the $L$-value appearing in Watson's triple product formula and the other on bounding shifted convolution sums, and which complement each other in a remarkable way to produce the full result. In this paper we extend Holowinsky and Soundararajan's methods to prove QUE for holomorphic Hecke eigenforms on $G L_{2}$ over a totally real number field, or more generally for automorphic forms of cohomological type on $G L_{2}$ over an arbitrary number field and which satisfy the Ramanujan bounds. For simplicity, we assume our fields to have narrow class number one throughout the paper, but this is not essential and can be avoided by working adelically as in the work of Nelson [29].

1.1. Structure of the paper. We introduce the manifolds and automorphic forms with which we shall work in section 2, before stating our results in section 3, We describe the structure of the proof in section 4 As our proof is a direct generalisation of the methods used by Holowinsky and Soundararajan over $\mathbb{Q}$, we do this by first giving an overview of their proof before explaining the modifications which must be made to extend it to a number field. Sections 5 to 7 contain the generalisation of Holowinsky's method of shifted convolution sums, and section 8 contains the extension of Soundararajan's approach of triple product identities and weak subconvexity. In section 9 we combine these two approaches to establish our main result, and in section 10 we prove the generalisation of Rudnick's theorem on the equidistribution of zero divisors of holomorphic forms. Section 11 is an appendix which contains various computations which are needed in the course of the proofs.

\section{Definitions AND NOTATION}

2.1. Arithmetic manifolds. We begin by introducing the manifolds on which we shall work. Let $F$ be a number field of narrow class number one with discriminant $D$ and regulator $R$. Let $F$ have degree $n$ and $r$ infinite places, of which $r_{1}$ are real and $r_{2}$ are complex. Let $\mathbb{F}=F \otimes_{\mathbb{Q}} \mathbb{R}$, and let $\mathbb{F}^{+}$be the subset of totally positive elements. If $\mathcal{O}$ is the ring of integers of $F$, let $\mathcal{O}^{+}=\mathcal{O} \cap \mathbb{F}^{+}$. We shall denote the multiplicative group of $\mathbb{F}^{+}$by $\mathbb{F}_{+}^{\times}$, and the group of totally positive units by $\mathcal{O}_{+}^{\times}$. Define $\mu_{+}$to be the group of totally positive roots of unity in $F$, which is the ordinary unit group if $F$ is totally complex and trivial otherwise.

Let $G_{i}=G L^{+}(2, \mathbb{R})$ for $i \leq r_{1}$ and $G L(2, \mathbb{C})$ otherwise, and $G=G_{1} \times \ldots \times G_{r}=$ $G L^{+}(2, \mathbb{F}) . Z_{i}$ will denote the centre of $G_{i}$, and $\overline{G_{i}}=G_{i} / Z_{i} . N$ will denote the usual unipotent subgroup of $G$ and $\bar{G}$, and $A$ and $M$ the maximal split and compact diagonal subgroups with lower entry equal to 1 . $K=K_{1} \times \ldots \times K_{r}$ will be the maximal compact. Let $\Gamma=G L^{+}(2, \mathcal{O})$ be the integral matrices with totally positive 
determinant, and define $\Gamma_{\infty}=\Gamma \cap B$ and $\Gamma_{U}=\Gamma \cap U$, where $B$ and $U$ are the upper triangular Borel and unipotent subgroups of $G L_{2}(F)$.

Let $\mathbb{H}_{F}=\bar{G} / K$ be identified with the product $\left(\mathbb{H}^{2}\right)^{r_{1}} \times\left(\mathbb{H}^{3}\right)^{r_{2}}$ of two and three dimensional hyperbolic spaces, and introduce on it the following coordinates:

$$
\begin{array}{rlr}
z & =\left(z_{1}, \ldots, z_{r}\right), \\
z_{t} & = \begin{cases}x_{t}+i y_{t}, \quad x_{t}, y_{t} \in \mathbb{R} & \text { for } t \leq r_{1}, \\
x_{t}+j y_{t}, \quad x_{t} \in \mathbb{C}, y_{t} \in \mathbb{R} & \text { for } t>r_{1},\end{cases} \\
x & =\left(x_{1}, \ldots, x_{r}\right) \in \mathbb{F}, & \\
y & =\left(y_{1}, \ldots, y_{r}\right) \in \mathbb{R}_{+}^{r} .
\end{array}
$$

We let

$$
d v=\bigwedge_{t \leq r_{1}} y_{t}^{-2} d x_{t} d y_{t} \wedge \bigwedge_{t>r_{1}} \frac{y_{t}^{-3}}{2 i} d x_{t} d \bar{x}_{t} d y_{t}
$$

be the product of standard hyperbolic measures on $\mathbb{H}_{F}$. We define $X=\Gamma \backslash \bar{G}$ and $Y=\Gamma \backslash \mathbb{H}_{F}$, so that automorphic forms on $G L_{2} / F$ of full level are equivalent to Hecke eigenforms on $X$.

Throughout the paper, we will use a multi-index notation for coordinates on $\mathbb{H}_{F}$ and the weights of automorphic forms; for instance, if $y$ is the coordinate on $\mathbb{H}_{F}$ introduced above and $k$ is an $r$-tuple of integers, the expression $y^{k}$ will denote $\prod y_{i}^{k_{i}}$. If $\delta_{i}$ is defined to be 1 for $i \leq r_{1}$ and 2 otherwise, for any $r$-tuple $x$ we denote $\prod x_{i}^{\delta_{i}}$ by $N x$, and the maximum of $\left|x_{i}\right|$ by $\|x\|$. If $a=\left(a_{i}\right)$ and $b=\left(b_{i}\right)$ are two elements of $\mathbb{F}$, the inequality $a>b$ will mean that $a_{i}>b_{i}$ when $\nu_{i}$ is a real place, and that $\left|a_{i}\right|>\left|b_{i}\right|$ when $\nu_{i}$ is complex. In particular, the set $\{x \in \mathcal{O}: x>0\}$ is just $\mathcal{O}^{+}$.

2.2. Eisenstein series. In addition to the usual complete Eisenstein series, we will work with two kinds of incomplete Eisenstein series which we term 'pure incomplete Eisenstein series' and 'unipotent Eisenstein series'. To define them, we must introduce the multiplicative characters of the group $\mathbb{F}_{+}^{\times} / \mathcal{O}_{+}^{\times}$following Hecke. Let $\left\{\epsilon_{j}: 1 \leq j \leq r-1\right\}$ be a generating set for $\mathcal{O}_{+}^{\times}$, and denote the image of $\epsilon_{j}$ at the $i$ th Archimedean place of $F$ by $\epsilon_{j}^{i}$. Define the matrix $A$ to be

$$
A=\left(\begin{array}{cccc}
1 / n & \log \left|\epsilon_{1}^{1}\right| & \ldots & \log \left|\epsilon_{r-1}^{1}\right| \\
\vdots & & & \\
1 / n & \log \left|\epsilon_{1}^{r}\right| & \ldots & \log \left|\epsilon_{r-1}^{r}\right|
\end{array}\right)
$$

and denote its inverse by

$$
A^{-1}=\left(\begin{array}{cccc}
1 & 1 & \ldots & 2 \\
e_{1}^{1} & e_{2}^{1} & \ldots & e_{r}^{1} \\
\vdots & & & \\
e_{1}^{r-1} & e_{2}^{r-1} & \ldots & e_{r}^{r-1}
\end{array}\right)
$$


where the first row of $A^{-1}$ contains $r_{1} 1$ 's and $r_{2} 2$ 's. We may now define the characters $\lambda_{m}(y)$ for $m \in \mathbb{Z}^{r-1}$ by the following formula:

$$
\begin{aligned}
\lambda_{m}: y=\left(y_{1}, \ldots, y_{r}\right) \in \mathbb{F}_{+}^{\times} & \mapsto \prod_{p=1}^{r} \prod_{q=1}^{r-1}\left|y_{p}\right|^{2 \pi i m_{q} e_{p}^{q}} \\
& =\exp \left(\sum_{p=1}^{r} \beta(m, p) \log \left|y_{p}\right|\right), \\
\text { where } \beta(m, p) & =2 \pi i \sum_{q=1}^{r-1} m_{q} e_{p}^{q} .
\end{aligned}
$$

As $\lambda_{m}$ is invariant under the action of $\mathcal{O}_{+}^{\times}$on $\mathbb{F}_{+}^{\times}$, it may be extended to a Hecke character on $F$ via the isomorphism $\mathbb{F}^{\times} / \mathcal{O}^{\times} \simeq \mathbb{F}_{+}^{\times} / \mathcal{O}_{+}^{\times}$. When defining Eisenstein series, we shall frequently think of $\lambda_{m}$ as a character of the multiplicative group $\left(\mathbb{R}_{+}^{\times}\right)^{r} / \mathcal{O}_{+}^{\times}$.

Having defined $\lambda_{m}$, we may let $E(s, m, z)$ denote the usual Eisenstein series associated to the character $N y^{s} \lambda_{m}(y)$ of the cusp of $X$. The pure incomplete Eisenstein series are formed by automorphising a function on $\Gamma_{\infty} \backslash \mathbb{H}_{F}$ which is invariant under $U$ and transforms according to $\lambda_{m}$ under the norm one elements of the diagonal. They are specified by an index $m \in \mathbb{Z}^{r-1}$ and a function $\psi \in C_{0}^{\infty}\left(\mathbb{R}^{+}\right)$ and are defined as

$$
E(\psi, m \mid z)=\sum_{\gamma \in \Gamma_{\infty} \backslash \Gamma} \psi(N y(\gamma z)) \lambda_{m}(y(\gamma z)) .
$$

The unipotent Eisenstein series are formed by automorphising a function on $\Gamma_{U} \backslash \mathbb{H}_{F}$ which is only invariant under $U$. They are specified by a function $g \in$ $C_{0}^{\infty}\left(\mathbb{R}_{+}^{r}\right)$ and defined as

$$
E(g \mid z)=\sum_{\gamma \in \Gamma_{U} \backslash \Gamma} g(y(\gamma z)) .
$$

We note that it is less standard to form Eisenstein series by symmetrising a function over $\Gamma_{U} \backslash \Gamma$ in this way, and while these series do not play a major part in the proof, their appearance is related to the key fact that the correct way in which to generalise Holowinsky's methods is by unfolding over the unipotent, as will be discussed in section 4.2 .

2.3. Representation theory of $S L(2, \mathbb{C})$. For $m \in \mathbb{N}$, let $\rho_{m}$ denote the irreducible $(m+1)$-dimensional representation of $S U(2) \subset S L(2, \mathbb{C})$ with Hermitian inner product $\langle$,$\rangle , and let *^{*}$ denote the associated conjugate linear isomorphism between $\rho_{m}$ and $\rho_{m}^{*}$. We choose an orthonormal basis $\left\{v_{t}\right\}(t=m, m-2, \ldots,-m)$ for $\rho_{m}$ and dual basis $\left\{v_{t}^{*}\right\}$ for $\rho_{m}^{*}$, consisting of eigenvectors of $M$ satisfying

$$
\left(\begin{array}{cc}
e^{i \theta} & 0 \\
0 & e^{-i \theta}
\end{array}\right) v_{t}=e^{i t \theta} v_{t}, \quad\left(\begin{array}{cc}
e^{i \theta} & 0 \\
0 & e^{-i \theta}
\end{array}\right) v_{t}^{*}=e^{-i t \theta} v_{t}^{*} .
$$

If $r \in \mathbb{C}$ and $k \in \mathbb{Z}$, let $I_{(k, r)}$ be the representation of $S L(2, \mathbb{C})$ unitarily induced from the character

$$
\chi:\left(\begin{array}{cc}
z & x \\
0 & z^{-1}
\end{array}\right) \mapsto(z /|z|)^{k}|z|^{2 i r} .
$$


These are unitarisable for $(k, r)$ in the set

$$
U=\{(k, r) \mid r \in \mathbb{R}\} \cup\{(k, r) \mid k=0, r \in i(-1,1)\},
$$

and two such representations $I_{(k, r)}, I_{\left(k^{\prime}, r^{\prime}\right)}$ are equivalent iff $(k, r)= \pm\left(k^{\prime}, r^{\prime}\right)$. Furthermore, these are all the irreducible unitary representations of $S L(2, \mathbb{C})$ other than the trivial representation. We choose a set $U^{\prime} \subset U$ representing every equivalence class in $U$ to be

$$
U^{\prime}=\{(k, r) \mid r \in(0, \infty)\} \cup\{(k, r) \mid r=0, k \geq 0\} \cup\{(k, r) \mid k=0, r \in i(0,1)\} .
$$

Given $\pi \in S \widehat{L(2, \mathbb{C})}$ nontrivial, we shall say that $\pi$ has weight $k$ and spectral parameter $r$ if it is isomorphic to $I_{(k, r)}$ with $(k, r) \in U^{\prime}$. As we shall work on $G L_{2}$ with trivial central character, we may specify the Archimedean components of our automorphic representations by specifying their restrictions to $S L_{2}$. At complex places we shall use the parameters just introduced, and at real places we shall use the customary weight and spectral parameter.

2.4. Automorphic forms. We shall consider QUE for cuspidal automorphic forms $\pi$ on $G L_{2} / F$ of full level, trivial central character and cohomological type. This means that their local factors at real places are holomorphic discrete series of even weight, and the factors at complex places have even weight and spectral parameter 0 . In the notation of section 2.1 these correspond to automorphic forms on $X$ of the prescribed Archimedean type and which are eigenfunctions of the Hecke operators. Our assumptions on the class number of $F$ and the level of $\pi$ imply that $\pi$ is not dihedral.

We denote the weight of $\pi$ by an $r$-tuple $k=\left(k_{i}\right)$, and its normalised Hecke eigenvalues by $\lambda_{\pi}(\mathfrak{p})$. Define $\rho_{k}$ to be the representation

$$
\rho_{k}=\bigotimes_{i \leq r_{1}} \chi_{k_{i}} \otimes \bigotimes_{i>r_{1}} \rho_{k_{i}}
$$

of $K$, noting that, in the presence of complex places, $K$ will be nonabelian and $\rho_{k}$ will have dimension greater than one for most choices of the weight. As $\rho_{k}$ occurs as a $K$-type in the Archimedean component of $\pi$, there is an embedding $R_{\pi}$ in $\operatorname{Hom}_{K}\left(\rho_{k}, L_{\text {cusp }}^{2}(X)\right)$ corresponding to $\pi$. We may associate to $R_{\pi}$ a section $F_{k}$ of the principal bundle $X \times_{K} \rho_{k}^{*}$ on $Y$, where we recall that for a representation $\tau$ of $K, X \times_{K} \tau$ is the quotient of $X \times \tau$ by the right $K$-action

$$
(x, v) k=\left(x k, \tau(k)^{-1} v\right)
$$

so that sections of $X \times_{K} \tau$ may be thought of as sections of $X \times \tau$ satisfying

$$
\tau(k) v(x k)=v(x) .
$$

$F_{k}$ may be defined by the relation $R_{\pi}(v)(x)=\left(F_{k}(x), v\right)$ for $v \in \rho_{k}$ and $x \in X$, which may be unwound to give

$$
\begin{aligned}
F_{k}(x) & =\prod_{i>r_{1}}\left(k_{i}+1\right)^{-1 / 2} \sum_{t} R_{\pi}\left(v_{t}\right)(x) v_{t}^{*}, \\
\left|F_{k}(x)\right|^{2} & =\prod_{i>r_{1}}\left(k_{i}+1\right)^{-1} \sum_{t}\left|R_{\pi}\left(v_{t}\right)(x)\right|^{2},
\end{aligned}
$$

where $\left\{v_{t}\right\}$ is a basis of $M$-eigenvectors for $\rho_{k}$. Note that $\left|F_{k}(x)\right|^{2}$ descends to a function on $Y$. Alternatively, we may define $E_{k}$ to be the restriction to $\Gamma$ of the 
representation

$$
\left(\bigotimes_{i \leq r_{1}} \operatorname{Sym}^{k_{i}-2}\right) \otimes\left(\bigotimes_{i>r_{1}} \operatorname{Sym}^{k_{i} / 2-1} \otimes{\overline{\mathrm{Sym}^{k}}}^{k_{i} / 2-1}\right)
$$

of $G$, and let $V_{k}$ be the associated local system on $Y$, which we equip with a certain canonical positive definite norm. Then $F_{k}$ may be thought of as a harmonic 1-form which represents a cohomology class in $H^{1}\left(Y, V_{k}\right)$ (this is why $\pi$ is referred to as being of cohomological type). However, we will not use this point of view in this paper and shall only refer the reader to the book of Borel and Wallach 11 where correspondences of this kind are described in detail.

We wish to establish the equidistribution of the probability measures $\left|F_{k}\right|^{2} d v$ on $Y$, in generalisation of holomorphic QUE over $\mathbb{Q}$. Because the $K$-integrals of $\left|R_{\pi}\left(v_{t}\right)\right|^{2}$ are independent of $t$, we may let $v_{k} \in \rho_{k}$ be the vector of highest weight and think of the measure $\left|F_{k}\right|^{2} d v$ as the pushforward of $\left|R_{\pi}\left(v_{k}\right)\right|^{2} d x$ from $X$. In the case where $F$ is totally real, the reader may instead let $f$ be a holomorphic Hecke eigenform with associated representation $\pi$, and let $F_{k}$ be the mass function $F_{k}=y^{k / 2} f$. In particular, the results stated in the next section may all be read with this simpler definition in mind.

To simplify the transition from Fourier expansions to shifted convolution sums in the next chapter, we will write the Fourier expansions of all our automorphic forms using sums over the ring of integers $\mathcal{O}$ rather than the inverse different $\mathcal{O}^{*}$ as follows:

$$
\phi(z)=\sum_{\xi \in \mathcal{O}} a_{\xi}(y) e(\operatorname{tr}(\xi \kappa x))
$$

where $\kappa$ will denote a fixed totally positive generator of $\mathcal{O}^{*}$ throughout. As the $F_{k}$ are vector-valued, it turns out that they may be expanded in Fourier series more simply by enlarging their domain $\mathbb{H}_{F}$, in a manner which we now describe. We identify $\mathbb{H}_{F}$ with the subgroup $N A$ of $G$ in the standard Iwasawa factorisation, and let $\mathbb{H}_{F}^{\prime}$ be the subgroup $N A M$. We then have an inclusion of $\mathbb{H}_{F}$ in $\mathbb{H}_{F}^{\prime}$, and we extend our hyperbolic coordinate system to $\mathbb{H}_{F}^{\prime}$ by allowing $y_{i}$ to take complex values for $i>r_{1}$. The $K$-covariance of $F_{k}$ means that it is determined by its values on $\mathbb{H}_{F}^{\prime}$, and these determine the embedding $R_{\pi}$ by the formula

$$
R_{\pi}(v)(g)=\left(\rho(k) v, F_{k}(z)\right),
$$

where $g=z k$ is the Iwasawa factorisation of $g$. On $\mathbb{H}_{F}^{\prime}$, we may expand $F_{k}$ in a Fourier series as

$$
F_{k}(z)=\sum_{\xi>0} a_{f}(\xi) \mathbf{K}_{k}(\xi \kappa y) e(\operatorname{tr}(\xi \kappa x)),
$$

where $\mathbf{K}_{k}(y)=\bigotimes_{t=1}^{r} \mathbf{K}_{t}\left(y_{t}\right)$ and the $\mathbf{K}_{t}\left(y_{t}\right)$ are defined by

$$
\begin{aligned}
& \mathbf{K}_{t}\left(y_{t}\right)=\left(y_{t}\right)^{k_{t} / 2} \exp \left(-2 \pi y_{t}\right) \quad \text { for } t \leq r_{1}, \\
& \mathbf{K}_{t}\left(y_{t}\right)=\left|y_{t}\right|^{k_{t} / 2+1} \sum_{j=0}^{k_{t}}\left(\begin{array}{c}
k_{t} \\
j
\end{array}\right)^{1 / 2} K_{k_{t} / 2-j}\left(4 \pi\left|y_{t}\right|\right) e^{\left(k_{t}-2 j\right) i \theta_{t} / 2} v_{k_{t}-2 j}^{*}, \quad t>r_{1},
\end{aligned}
$$

and $\theta_{t}$ is the argument of $y_{t}$. The formula for the Whittaker functions $\mathbf{K}_{t}$ at complex places is taken from Jacquet-Langlands [19]. The coefficients $a_{f}(\xi)$ are proportional to the Hecke eigenvalues $\lambda_{\pi}(\xi)$,

$$
a_{f}(\xi)=\lambda_{\pi}(\xi) N \xi^{-1 / 2} a_{f}(1),
$$


and the first Fourier coefficient is determined by the $L^{2}$ normalisation of $F_{k}$ to be

$$
\left|a_{f}(1)\right|^{2}=\frac{2^{7 r_{2}-1} \pi^{r_{1}+3 r_{2}}}{|D| L\left(1, \operatorname{sym}^{2} \pi\right)} \prod_{i \leq r_{1}} \frac{(4 \pi)^{k_{i}}}{\Gamma\left(k_{i}\right)} \prod_{i>r_{1}} \frac{(2 \pi)^{k_{i}}}{\Gamma\left(k_{i} / 2+1\right)^{2}} .
$$

(See section 11.2 for this calculation.)

\section{Statement of RESUlts}

Our main result is Theorem 1, which establishes QUE for the sections $F_{k}$ under the assumption that the associated cohomological representations $\pi$ satisfy the Ramanujan bound; this is known when $F$ is totally real or imaginary quadratic, as discussed below. We must also assume that the weight $k=\left(k_{i}\right)$ of $\pi$ tends to infinity in a weaky uniform way, namely that there exists $\nu>0$ such that $k_{i} \geq\|k\|^{\nu}$ for all $i$.

Theorem 1. If $\phi$ is a Hecke-Maass cusp form, we have

$$
\left|\left\langle\phi F_{k}, F_{k}\right\rangle\right| \ll_{\phi, \epsilon, \nu}(\log \|k\|)^{-1 / 30+\epsilon} .
$$

If $\phi$ is a pure incomplete Eisenstein series, we have

$$
\left\langle\phi F_{k}, F_{k}\right\rangle=\frac{1}{\operatorname{Vol}(Y)}\langle\phi, 1\rangle+O_{\phi, \epsilon, \nu}\left((\log \|k\|)^{-2 / 15+\epsilon}\right) .
$$

Let us give two simple illustrations of the content of this theorem in the cases where Ramanujan is known. Suppose that $F$ is totally real and $\Gamma=G L^{+}(2, \mathcal{O})$ is the subgroup of $G L(2, \mathcal{O})$ of elements with totally positive determinant. Fix $\nu>0$, and let $\left\{f_{n}\right\}$ be a sequence of holomorphic Hecke modular forms for $\Gamma$ whose weights $k_{n}=\left(k_{i, n}\right)$ satisfy $k_{i, n} \geq\|k\|^{\nu}$ for all $i$. Theorem 1 implies

Corollary 2. The normalised Petersson probability measures $\mu_{n}=y^{k_{n}}\left|f_{n}(z)\right|^{2} d v$ tend weakly to the uniform measure on $\Gamma \backslash\left(\mathbb{H}^{2}\right)^{n}$ as $k \rightarrow \infty$.

An equidistribution theorem of this kind for Hilbert modular forms was first conjectured by Liu in 22. As a consequence of Corollary 2] we prove that if $k$ is a fixed positive weight and $\left\{f_{T}\right\}$ a sequence of holomorphic Hecke forms of weight $T k=\left(T k_{1}, \ldots, T k_{n}\right)$, then the zero divisors $Z_{T}$ of $f_{T}$ become equidistributed on $\Gamma \backslash\left(\mathbb{H}^{2}\right)^{n}$ as $T \rightarrow \infty$, either as Lelong $(1,1)$-currents or as measures defined by integration over $Z_{T}$ with respect to the volume form of the induced Riemannian metric. This result is stated more fully in section 3.1, and generalises a theorem of Rudnick 31 on the equidistribution of zeros of Hecke modular forms on $S L(2, \mathbb{Z})$.

In the imaginary quadratic case, let $Y$ be the Bianchi manifold $\Gamma \backslash \mathbb{H}^{3}$, where $\mathcal{O}$ is the ring of integers in an imaginary quadratic field $F$ and $\Gamma=S L(2, \mathcal{O})$. Theorem 1 proves the equidistribution of a measure $\mu_{\pi}$ on $Y$ which may be associated to any cuspidal automorphic form $\pi$ of cohomological type on $\Gamma \backslash S L(2, \mathbb{C})$. If $\phi \in \pi$ is a vector of minimal $K$-type, we may define $\mu_{\pi}$ to be the pushforward of $|\phi|^{2} d g$ to $Y$. Alternatively, we may define $E_{d}$ to be the representation $\mathrm{Sym}^{d} \otimes \overline{\mathrm{Sym}}^{d}$ of $S L(2, \mathbb{C})$ as in section 2.4 , and let $V_{d}$ be the associated local system on $Y$ which we equip with a certain canonical positive definite norm. If $\pi$ has weight $k=2 d+2, \phi$ corresponds to a 1-form $\omega \in A^{1}\left(Y, V_{d}\right)$ which is harmonic with respect to the norm on $V_{d}$ and is an eigenform for the Hecke operators. If we define the norm $\|\cdot\|$ on $T^{*} Y \otimes V_{d}$ to be the tensor product of the Riemannian norm on $T^{*} Y$ and the norm on $V_{d}$ mentioned earlier, and $d v$ is the hyperbolic volume, it may be shown that $\mu_{\pi}$ 
is equal to $\|\omega\|^{2} d v$. Theorem 1 then implies

Corollary 3. If $\left\{\pi_{n}\right\}$ is a sequence of cohomological automorphic forms on $\Gamma \backslash S L(2, \mathbb{C})$ whose weights are tending to $\infty$, then the associated measures $\mu_{\pi_{n}}$ tend weakly to the hyperbolic volume on $Y$.

Theorem 1 is proven by combining the following two results, which summarise the extensions of Holowinsky and Soundararajan's respective approaches to proving the equidistribution of $F_{k}$. Their statements are almost identical to those of the original theorems over $\mathbb{Q}$, which are recalled in section 4.1, with the only significant difference being that we must impose our assumption on the uniform growth of the weight in Theorem 4.

Theorem 4. Fix an automorphic form $\phi$, and suppose that there exists a $\nu>0$ such that $k_{i}>\|k\|^{\nu}$ for all $i$. Define

$$
M_{k}(\pi)=\frac{1}{(\log \|k\|)^{2} L\left(1, \operatorname{sym}^{2} \pi\right)} \prod_{N \mathfrak{p} \leq\|k\|}\left(1+\frac{2\left|\lambda_{\pi}(\mathfrak{p})\right|}{N \mathfrak{p}}\right) .
$$

If $\phi$ is a Hecke-Maass cusp form, then

$$
\left\langle\phi F_{k}, F_{k}\right\rangle \ll_{\phi, \epsilon, \nu}(\log \|k\|)^{\epsilon} M_{k}(\pi)^{1 / 2}
$$

for any $\epsilon>0$. If $\phi$ is a pure incomplete Eisenstein series, then

$$
\left\langle\phi F_{k}, F_{k}\right\rangle=\frac{1}{\operatorname{Vol}(Y)}\langle\phi, 1\rangle+O_{\phi, \epsilon, \nu}\left((\log \|k\|)^{\epsilon} M_{k}(\pi)^{1 / 2}\left(1+R_{k}(\pi)\right)\right)
$$

for any $\epsilon>0$, where

$$
R_{k}(\pi)=\frac{1}{\sqrt{N k} L\left(1, \operatorname{sym}^{2} \pi\right)} \sum_{m} \int_{-\infty}^{+\infty} \frac{\left|L\left(1 / 2+i t, \operatorname{sym}^{2} \pi \otimes \lambda_{-m}\right)\right|}{(|t|+\|m\|+1)^{A}} d t .
$$

Theorem 5. If $\phi$ is a Hecke-Maass cusp form, we have

$$
\left|\left\langle\phi F_{k}, F_{k}\right\rangle\right| \ll_{\phi, \epsilon} \frac{(\log \|k\|)^{-1 / 2+\epsilon}}{L\left(1, \operatorname{sym}^{2} \pi\right)} .
$$

If $E\left(\frac{1}{2}+i t, m, \cdot\right)$ is a unitary Eisenstein series, we have

$$
\left|\left\langle E\left(\frac{1}{2}+i t, m, \cdot\right) F_{k}, F_{k}\right\rangle\right| \ll_{\epsilon}(1+|t|+\|m\|)^{2 n} \frac{(\log \|k\|)^{-1+\epsilon}}{L\left(1, \operatorname{sym}^{2} \pi\right)} .
$$

We shall prove Theorem 4 in sections 5 to 7 and Theorem 5 in section 8 , before combining them to give our main result in section 9. The presence of these two components and the way in which they interact makes the overall proof somewhat elaborate, and so we begin by reviewing its basic outline in the case of $S L(2, \mathbb{Z})$ and giving an overview of our modifications in section 4 . Our assumption that $\pi$ satisfies the Ramanujan bound is needed in the proofs of both Theorems 4 and 5 , in the first case to establish the weak form of Ramanujan required by Soundararajan's weak subconvexity theorem, and in the second case as an ingredient in bounding shifted convolution sums. It is known when $F$ is totally real or imaginary quadratic, and so we have an unconditional theorem in these cases. In the totally real case this is derived from Deligne's theorem by Blasius in [3, while in the imaginary quadratic case this relies on deep work of Harris, Soudry, Taylor, Berger, Harcos 
et al. [2, 12 and requires the construction of a theta lift from $G L_{2} / F$ to $G S p_{4} / \mathbb{Q}$, where complex geometry is available. The generalisation of their results to other fields with complex places is not yet established, and consequently we have no unconditional result outside totally real and imaginary quadratic fields. On the other hand, Ramanujan will hold for forms lifted from totally real subfields and so our theorem becomes unconditional if the family of cohomological forms of fixed level has the structure suggested by the results of [7], i.e. if base change and CM constructions account for all but finitely many forms.

The assumption we have made on the uniform growth of the weight is a purely technical one. Nelson [29] shows that it may be removed in the case when $F$ is totally real, and by combining the triple product identities of section 8.1 with the Lindelöf hypothesis we see that it should be unnecessary in general. The reason we have adopted it is so that when we come to the point in the generalisation of Holowinsky's theorem at which we apply the sieve, it will ensure that we are sieving over a rounded subset of the ring of integers rather than a narrow box. Nelson's improvement relies on an explicit formula for the integral appearing as a weight in the shifted convolution sum (32) of section [5.3, and it would be interesting to know if this can be extended to the case of one or more complex places.

Theorem 1 establishes QUE for any sequence of sections $F_{k}$ over a totally real or imaginary quadratic field whose weights tend to infinity with the required uniformity. However, we should ask whether such a sequence exists for these fields. When $F$ is totally real, Riemann-Roch ensures that the dimension of the space $S_{k}$ of holomorphic cusp forms of weight $k$ is $\sim N k$, with an exact formula established by Shimizu in 36]. Over a general field, base change from $\mathbb{Q}$ is expected to provide $\sim k$ forms of parallel weight $k$ on a sufficiently deep congruence subgroup of $\Gamma$, where the term 'parallel' means that the weights at all places are equal as in the totally real case. In particular, for $F$ imaginary quadratic it has been proven by Finis, Grunewald and Tirao [7] that base change produces forms of full level and so our result is not vacuous for the Bianchi manifolds. The proof may be easily modified to allow a nontrivial level in any case, so by restricting to forms base changed from $\mathbb{Q}$ (or another totally real subfield) which are known to satisfy Ramanujan, we may view it as having content over any solvable field $F$.

3.1. Equidistribution of zero currents. One consequence of QUE for holomorphic modular forms over $\mathbb{Q}$ is that the zeros of a sequence of forms become equidistributed with respect to hyperbolic measure as $k \rightarrow \infty$, as was proven by Shiffman and Zelditch [35] for compact hyperbolic surfaces and extended to $S L(2, \mathbb{Z}) \backslash \mathbb{H}^{2}$ by Rudnick 31. Using their methods, we have derived the analogous statement about the equidistribution of the zero divisors of holomorphic modular forms from our proof of holomorphic QUE. We may prove this equidistribution either in the sense of measures of integration over the (smooth parts of the) zero divisors, or in the more refined sense of Lelong (1,1)-currents, which we now describe.

As we shall only consider totally real number fields from this point until the end of section [5] in these sections we shall use $\mathbb{H}^{n}$ to denote the product of $n$ copies of the upper half-plane, so that the holomorphic forms $f$ we consider live on $Y=\Gamma \backslash \mathbb{H}^{n}$. In higher dimensions we may replace the sum of delta measures at the zeros of $f$ by the current of integration over its zero divisor $Z_{f}$, which is a distribution on differential forms of bidegree $(n-1, n-1)$. If $Z_{f}=\sum_{i} \operatorname{ord}_{V_{i}}(f) V_{i}$ 
is the expression of $Z_{f}$ as the sum of irreducible subvarieties, then

$$
\left(Z_{f}, \phi\right)=\sum_{i} \operatorname{ord}_{V_{i}}(f) \int_{V_{i}} \phi
$$

for all smooth, compactly supported forms $\phi$ on $\Gamma \backslash \mathbb{H}^{n}$. To define these notions in the presence of torsion in $\Gamma$, we use the standard procedure of choosing $\Gamma^{\prime} \subset \Gamma$ to be of finite index and torsion free, and defining forms, subvarieties etc. on $\Gamma \backslash \mathbb{H}^{n}$ to be those on $\Gamma^{\prime} \backslash \mathbb{H}^{n}$ which are invariant under $\Gamma$. Integrals such as (10) are defined to be the lifted integral on $\Gamma^{\prime} \backslash \mathbb{H}^{n}$ divided by $\left|\Gamma^{\prime}: \Gamma\right|$. We shall use $\stackrel{w^{*}}{\longrightarrow}$ to denote weak-* convergence of currents. With these notions in mind, we may state our result.

Theorem 6. Fix a weight $k=\left(k_{i}\right), k_{i}>0$, and let $\left\{f_{T}\right\}$ be a sequence of holomorphic Hecke modular forms of weight $T k=\left(T k_{i}\right)$. Define

$$
\begin{aligned}
\omega & =\frac{-i}{2 \pi} \partial \bar{\partial} \log y^{k} \\
& =\frac{1}{4 \pi} \sum k_{i} y_{i}^{-2} d x_{i} \wedge d y_{i} .
\end{aligned}
$$

If $Z_{T}$ are the zero divisors of $f_{T}$, then $\frac{1}{T} Z_{T} \stackrel{w^{*}}{\longrightarrow} \omega$, i.e.

$$
\lim _{T \rightarrow \infty}\left(\frac{1}{T} Z_{T}, \phi\right)=\int_{Y} \omega \wedge \phi
$$

for all continuous, compactly supported $(n-1, n-1)$-forms $\phi$. In particular, if $k=(2, \ldots, 2)$, then $\frac{1}{T} Z_{T} \stackrel{w^{*}}{\longrightarrow} \omega_{0}$, the Kähler form of $Y$ with the product hyperbolic metric.

This theorem is based on ideas from complex potential theory as developed for problems in quantum chaos in [30, 31, 35. It may be loosely interpreted as saying that not only do the (smooth parts of the) submanifolds $Z_{T}$ become equidistributed as measures of integration with respect to the induced Riemannian volume, but that the directions in which their tangent subspaces lie are also becoming equidistributed. We prove Theorem 6 in section 10.

\section{Outline of the PROOF}

4.1. The proof over $\mathbb{Q}$. We begin by giving an outline of Holowinsky and Soundararajan's proof over $\mathbb{Q}$, as our proof over a number field runs on the same lines as theirs. Suppose $f$ is a holomorphic Hecke eigenform of weight $k$ on $Y=S L(2, \mathbb{Z}) \backslash \mathbb{H}$, with associated mass function $F_{k}=y^{k / 2} f$. We wish to show that the normalised probability measure $\mu_{f}=\left|F_{k}\right|^{2} y^{-2} d x d y$ tends weakly to the hyperbolic measure $\frac{3}{\pi} y^{-2} d x d y$ as $k$ tends to infinity, i.e. that for all $h \in C_{0}^{\infty}(X)$,

$$
\mu_{f}(h)=\int_{Y} h\left|F_{k}\right|^{2} y^{-2} d x d y \rightarrow \frac{3}{\pi}\langle h, 1\rangle .
$$

In 15, 16, 40, Holowinsky and Soundararajan have established this by decomposing $h$ in two different bases for smooth functions on $X$, the first a complete set of eigenfunctions for the Laplacian and the second the incomplete Poincaré series $P_{m}$, defined by

$$
P_{m}(\psi \mid z)=\sum_{\gamma \in \Gamma_{\infty} \backslash \Gamma} e(m x(\gamma z)) \psi(y(\gamma z))
$$


for $m \in \mathbb{Z}$ and $\psi \in C_{0}^{\infty}\left(\mathbb{R}^{+}\right)$. The chosen basis of Laplace eigenfunctions consists of the constant function, Hecke-Maass cusp forms $\phi$ and unitary Eisenstein series $E\left(\frac{1}{2}+i t, \cdot\right)$, and the corresponding integrals which must be estimated are $\left\langle\phi F_{k}, F_{k}\right\rangle$ and $\left\langle E\left(\frac{1}{2}+i t, \cdot\right) F_{k}, F_{k}\right\rangle$. These integrals may be expressed in terms of central $L$ values, using Watson's formula in the first case and the classical Rankin-Selberg formula in the second, and so one may hope that the theory of $L$-functions would provide nontrivial upper bounds for them. The convex bound just fails to be of use here; however, by strengthening the convex bound by a factor of $(\log C)^{-1+\epsilon}$, where $C$ is the analytic conductor, Soundararajan obtains the following result:

Theorem 7. If $\phi$ is a Hecke-Maass cusp form, we have

$$
\left|\left\langle\phi F_{k}, F_{k}\right\rangle\right| \ll_{\phi, \epsilon} \frac{(\log k)^{-1 / 2+\epsilon}}{L\left(1, \operatorname{sym}^{2} f\right)} .
$$

If $E\left(\frac{1}{2}+i t, \cdot\right)$ is a unitary Eisenstein series, we have

$$
\left|\left\langle E\left(\frac{1}{2}+i t, \cdot\right) F_{k}, F_{k}\right\rangle\right| \ll_{\epsilon}(1+|t|)^{2} \frac{(\log k)^{-1+\epsilon}}{L\left(1, \operatorname{sym}^{2} f\right)} .
$$

The equidistribution of $\mu_{f}$ would follow from Theorem 7 if one knew that $L\left(1, \operatorname{sym}^{2} f\right) \gg(\log k)^{-1 / 2+\delta}$ for some $\delta>0$. This is certainly expected, as it follows from the generalised Riemann hypothesis that $L\left(1, \operatorname{sym}^{2} f\right)$ is bounded below by a power of $\log \log k$. The best unconditional bound in this direction is due to Hoffstein and Lockhart [13, and Goldfeld, Hoffstein and Lieman [10, who prove that $L\left(1, \operatorname{sym}^{2} f\right) \gg(\log k)^{-1}$; this is a deep result analogous to proving that there is no Siegel zero. The bound $L\left(1, \operatorname{sym}^{2} f\right) \gg(\log k)^{-1 / 2+\delta}$ is known unconditionally for all but $K^{\epsilon}$ eigenforms of weight $\leq K$ by a zero density argument; however, one cannot yet rule out the existence of forms with small values of $L\left(1, \mathrm{sym}^{2} f\right)$ for which Soundararajan's approach is insufficient.

Holowinsky's approach is to test $\mu_{f}$ against incomplete Poincaré and Eisenstein series. This is equivalent to testing $\mu_{f}$ against Hecke-Maass cusp forms and incomplete Eisenstein series and evaluating the inner products $\left\langle\phi F_{k}, F_{k}\right\rangle$ by regularising them with a second incomplete Eisenstein series and then unfolding. In doing this one is led to estimating the shifted convolution sums

$$
\sum_{n \sim k} \lambda_{f}(n) \lambda_{f}(n+l)
$$

for fixed $l$ as $k \rightarrow \infty$, where $\lambda_{f}$ are the automorphically normalised Hecke eigenvalues of $f$. Quite strikingly, Holowinsky is able to obtain useful bounds for these by taking absolute values of the terms and forgoing any additive cancellation. The idea behind this is that the eigenvalues $\lambda_{f}(p)$ not only satisfy the Ramanujan bound $\left|\lambda_{f}(p)\right| \leq 2$, but are distributed in the interval $[-2,2]$ according to Sato-Tate measure and so on average $\left|\lambda_{f}(p)\right|$ will be significantly smaller than 2 (we do not need to consider dihedral forms as we are working at full level). Moreover, as a typical $\lambda_{f}(n)$ is a product of many $\lambda_{f}(p)$ 's this leads to a gain on average over the bound $\left|\lambda_{f}(n)\right| \leq \tau(n)$. This phenomenon may also be seen in the work of Elliott, Moreno and Shahidi [6] where they prove the bound

$$
\sum_{n \leq x}|\tau(n)| \ll x^{13 / 2}(\log x)^{-1 / 18},
$$


where $\tau$ here denotes Ramanujan's $\tau$-function. Holowinsky uses this idea, combined with a large sieve to show that $n$ and $n+l$ seldom both have small prime factors, to prove the following:

Theorem 8. If $\lambda_{f}$ are the normalised Hecke eigenvalues of $f$ as above, define

$$
M_{k}(f)=\frac{1}{(\log k)^{2} L\left(1, \operatorname{sym}^{2} f\right)} \prod_{p \leq k}\left(1+\frac{2\left|\lambda_{f}(p)\right|}{p}\right) .
$$

If $\phi$ is a Hecke-Maass cusp form, we have

$$
\left|\left\langle\phi F_{k}, F_{k}\right\rangle\right| \ll_{\phi, \epsilon}(\log k)^{\epsilon} M_{k}(f)^{1 / 2} .
$$

If $E(\psi \mid \cdot)$ is an incomplete Eisenstein series, we have

$$
\left|\left\langle E(\psi \mid \cdot) F_{k}, F_{k}\right\rangle-\frac{3}{\pi}\langle E(\psi \mid \cdot), 1\rangle\right| \ll_{\psi, \epsilon}(\log k)^{\epsilon} M_{k}(f)^{1 / 2}\left(1+R_{k}(f)\right),
$$

where

$$
R_{k}(f)=\frac{1}{k^{1 / 2} L\left(1, \operatorname{sym}^{2} f\right)} \int_{-\infty}^{\infty} \frac{\left|L\left(1 / 2+i t, \operatorname{sym}^{2} f\right)\right|}{(1+|t|)^{10}} d t .
$$

One can see the appeal to Sato-Tate in the quantity $M_{k}(f)$ appearing in Theorem 8 , if we only apply the bound $\left|\lambda_{f}(p)\right| \leq 2$ to this, one finds that $M_{k}(f) \ll$ $(\log k)^{2} L\left(1, \operatorname{sym}^{2} f\right)^{-1}$, which is of no use. However, under certain natural assumptions about the distribution of $\lambda_{f}(p)$ it may be shown that $M_{k}(f)$ is small; more precisely, in 14, Holowinsky shows that if neither $L\left(1, \operatorname{sym}^{2} f\right)$ nor $L\left(1, \operatorname{sym}^{4} f\right)$ are small, then we have $M_{k}(f) \ll(\log k)^{-\delta}$ for some $\delta>0$. As with Soundararajan's theorem, these assumptions may also be shown to hold for almost all eigenforms using zero density estimates.

Surprisingly, while both of these approaches may fail it can be shown that together they cover all cases completely. Intuitively speaking, if $L\left(1, \operatorname{sym}^{2} f\right)<$ $(\log k)^{-1 / 2+\delta}$ is small, then we should have $\lambda_{f}\left(p^{2}\right) \sim-1$ for most primes $p \leq k$ (a Siegel zero type phenomenon). However, $M_{k}(f)$ is proven in [16 to satisfy the upper bound

$$
M_{k}(f) \ll(\log k)^{\epsilon} \exp \left(-\sum_{p \leq k} \frac{\left(\left|\lambda_{f}(p)\right|-1\right)^{2}}{p}\right),
$$

and if $\lambda_{f}\left(p^{2}\right) \sim-1$, then $\lambda_{f}(p)^{2}-1 \sim-1$, so that $\lambda_{f}(p) \sim 0$ for most $p \leq k$ and the right-hand side of (11) should be small. The precise bound Holowinsky and Soundararajan prove based on this argument is

$$
M_{k}(f) \ll(\log k)^{1 / 6}(\log \log k)^{9 / 2} L\left(1, \operatorname{sym}^{2} f\right)^{1 / 2} .
$$

This inequality may be used to show that if $\phi$ is a cusp form and $L\left(1, \operatorname{sym}^{2} f\right)<$ $(\log k)^{-1 / 3-\delta}$ for some $\delta>0$, then $M_{k}(f)$, and hence $\left\langle\phi F_{k}, F_{k}\right\rangle$, is small. However, if $L\left(1, \operatorname{sym}^{2} f\right)>(\log k)^{-1 / 3-\delta}>(\log k)^{-1 / 2+\delta}$, then Theorem[7 shows that $\left\langle\phi F_{k}, F_{k}\right\rangle$ is small. This shows how Theorems 7 and 8 complement each other in the cusp form case, and a similar relationship holds between them in the incomplete Eisenstein case. 
4.2. Extension to a number field. We now describe the steps which we have taken to generalise the method of section 4.1 to a number field. Soundararajan's approach is the easier of the two to extend, as one has the triple product formula of Ichino [17 available to generalise Watson's formula, and Soundararajan's weak subconvexity theorem is sufficiently general to also be applicable to the central $L$-value which appears there. The only technical difficulty is in making Ichino's formula sufficiently quantitative, which requires estimating certain Archimedean integrals. The necessary computation at complex places was carried out in 24] using a result of Michel and Venkatesh appearing in [25], while at real places it may be obtained by comparison with Watson's formula. Applying weak subconvexity is then straightforward, with the only consideration being that Soundararajan's theorem is stated for $L$-functions over $\mathbb{Q}$ rather than a number field. However, it is easy to show that our $L$-functions still satisfy the required hypotheses when viewed as Euler products over $\mathbb{Q}$. These steps are carried out in section 8

The modifications which we have made to Holowinsky's approach are more substantial, and we shall now describe his method in detail before illustrating how we have adapted it in the simple case of a real quadratic field. Holowinsky's approach for $S L(2, \mathbb{Z})$ is similar to calculating the integral of $\left|F_{k}\right|^{2}$ against a Poincaré series in terms of shifted convolution sums. For a Hecke-Maass form or incomplete Eisenstein series $\phi$, he defines a regularised unfolding of $\left\langle\phi F_{k}, F_{k}\right\rangle$ in terms of a fixed positive $g \in C_{0}^{\infty}\left(\mathbb{R}^{+}\right)$and a slowly growing parameter $T$ by

$$
I_{\phi}(T)=\int_{\Gamma_{\infty} \backslash \mathbb{H}} g(T y) \phi(z)\left|F_{k}(z)\right|^{2} d \mu .
$$

This behaves like the integral of $\phi\left|F_{k}\right|^{2}$ over $T$ copies of a fundamental domain for $S L(2, \mathbb{Z})$, and this may be quantified by taking the Mellin transform $G$ of $g$ and expressing (12) in terms of Eisenstein series as

$$
I_{\phi}(T)=\frac{1}{2 \pi i} \int_{(\sigma)} G(-s) T^{s} \int_{Y} E(s, z) \phi(z)\left|F_{k}(z)\right|^{2} d \mu .
$$

Shifting the contour to $\sigma=1 / 2$ then gives

$$
\begin{aligned}
I_{\phi}(T) & =c T\left\langle\phi F_{k}, F_{k}\right\rangle+O\left(T^{1 / 2}\right), \\
\text { where } \quad c & =\frac{3}{\pi}\langle E(g \mid z), 1\rangle .
\end{aligned}
$$

Holowinsky then calculates $I_{\phi}(T)$ in a second way using the Fourier expansions of $\phi$ and $f$,

$$
\begin{aligned}
& f(z)=\sum_{n \geq 1} a_{f}(n) e(n z), \\
& \phi(z)=\sum_{l} a_{l}(y) e(l x) .
\end{aligned}
$$

Only those $l$ with $|l| \ll T^{1+\epsilon}$ make a significant contribution, and for those $l$ Holowinsky defines

$$
\begin{aligned}
S_{l}(T) & =\int_{\Gamma_{\infty} \backslash \mathbb{H}} g(T y) a_{l}(y) e(l x)\left|F_{k}(z)\right|^{2} d \mu \\
& \ll\left|a_{l}\left(T^{-1}\right)\right| \sum_{n \geq 1}\left|a_{f}(n) a_{f}(n+l)\right|\left(\int_{0}^{\infty} g(T y) y^{k-2} e^{-2 \pi(2 n+l) y} d y\right)
\end{aligned}
$$


so that

$$
I_{\phi}(T)=\sum_{|l| \ll T^{1+\epsilon}} S_{l}(T)+O\left(T^{1 / 2}\right) .
$$

When $l \neq 0$, the regularising factor $g(T y)$ effectively truncates the sum in (13) to $n \ll T k$, and we end up with an upper bound for $S_{l}(T)$ of

$$
S_{l}(T) \ll \frac{\left|a_{l}\left(T^{-1}\right)\right|}{k L\left(1, \operatorname{sym}^{2} f\right)} \sum_{n \leq T k}\left|\lambda_{f}(n) \lambda_{f}(n+l)\right| .
$$

The expected main term $\frac{3}{\pi}\langle\phi, 1\rangle$ appears in $S_{0}(T)$, and so to prove that $\frac{3}{\pi}\langle\phi, 1\rangle$ and $\left\langle\phi F_{k}, F_{k}\right\rangle$ are close one needs to bound the off-diagonal terms $S_{l}(T)$ and hence $\sum_{n \leq x}\left|\lambda_{f}(n) \lambda_{f}(n+l)\right|$. Having given up additive cancellation in this sum, Holowinsky instead proceeds by using the ideas discussed in section 4.1 to show that $\left|\lambda_{f}(n) \lambda_{f}(n+l)\right|$ is small on average.

We have extended this method to work over an arbitrary number field $F$, with the key innovation being the way the unfolding is carried out in the presence of units. For simplicity, we will briefly describe the method in the case of a real quadratic field $F=\mathbb{Q}(\sqrt{d})$, and $f$ a holomorphic Hecke modular form of parallel weight $(k, k)$ with associated automorphic representation $\pi$. Let $\phi$ be a Hecke-Maass cusp form, and write the Fourier expansions of $f$ and $\phi$ as

$$
\begin{aligned}
& f(z)=\sum_{\eta>0} a_{f}(\eta) e(\operatorname{tr}(\eta \kappa z)), \\
& \phi(z)=\sum_{\xi \neq 0} a_{\xi}(y) e(\operatorname{tr}(\xi \kappa x)) .
\end{aligned}
$$

The totally positive units $\mathcal{O}_{+}^{\times}$of $\mathcal{O}$ act on the terms of these expansions, and when unfolding we must do so in a way which breaks this symmetry so that the resulting shifted convolution sums are over well-rounded sets in $\mathcal{O}$. The correct approach is to unfold to $\Gamma_{U} \backslash \mathbb{H}^{2} \simeq \mathbb{R}_{+}^{2} \times\left(\mathbb{R}^{2} / \mathcal{O}\right)$ and localise in a set of the form $B_{T} \times\left(\mathbb{R}^{2} / \mathcal{O}\right)$, where $B$ is a ball in $\mathbb{R}_{+}^{2}$ and we multiply it by $T^{-1}$ in each coordinate to get $B_{T}$. This lets us largely ignore the units, and when we form the analogues of $S_{l}(T)$ it will allow us to truncate the resulting shifted convolution sum over $\mathcal{O}$ at each place separately. We therefore define $I_{\phi}(T)$ as the integral

$$
I_{\phi}(T)=\int_{\Gamma_{U} \backslash \mathbb{H}^{2}} g(T y) \phi(z)\left|F_{k}(z)\right|^{2} d v,
$$

where now we let $h \in C_{0}^{\infty}\left(\mathbb{R}^{+}\right)$be a positive function and $g \in C_{0}^{\infty}\left(\mathbb{R}_{+}^{2}\right)$ be its square. We extract a main term $c T^{2}\left\langle\phi F_{k}, F_{k}\right\rangle$ from this as before, by forming the symmetrised function

$$
\widetilde{g}(y)=\sum_{u \in \mathcal{O}_{+}^{\times}} g(u y)
$$

and expanding it in the multiplicative characters of $\mathbb{R}_{+}^{2} / \mathcal{O}_{+}^{\times}$to express $I_{\phi}(T)$ in terms of integrals against Eisenstein series. When we calculate $I_{\phi}(T)$ in terms of the Fourier expansion of $\phi$ it may again be shown that only those $\xi$ with $\|\xi\| \ll T^{1+\epsilon}$ contribute, and for these we define

$$
S_{\xi}(T)=\int_{\Gamma_{U} \backslash \mathbb{H}^{2}} g(T y) a_{\xi}(y) \exp (2 \pi i \operatorname{tr}(\xi \kappa x))\left|F_{k}(z)\right|^{2} d v .
$$


The analogue of the upper bound (13) for $S_{\xi}(T)$ is

$$
S_{\xi}(T) \ll\left|a_{\xi}\left(T^{-1}\right)\right| \sum_{\eta>0}\left|a_{f}(\eta) a_{f}(\eta+\xi)\right| \int_{\mathbb{R}_{+}^{2}} g(T y) y^{k-2} \exp (2 \pi \operatorname{tr}((2 \eta+\xi) \kappa y)) d y .
$$

The key feature of the integral appearing here is that it factorises over the places of $\mathbb{Q}(\sqrt{d})$, and the value of each factor depends only on the image of $2 \eta+\xi$ at that place. This lets us truncate the sum to the ball of radius $k$ in $\mathcal{O}$ and leaves us with bounding $\sum_{0<\eta<k}\left|\lambda_{\pi}(\eta) \lambda_{\pi}(\eta+\xi)\right|$. This round set is well suited to the application of the large sieve for lattices in $\mathbb{R}^{n}$, and we may carry out Holowinsky's sieving approach as before by translating congruences modulo primes $\mathfrak{p}$ of $\mathcal{O}$ to sieve conditions in $\mathcal{O} / p \mathcal{O}$ without significant interference from the units.

We carry this method out in detail in sections 5 to 7 . The proof splits into two parts, the first of which is reducing bounds on $\left\langle\phi F_{k}, F_{k}\right\rangle$ to ones on shifted convolution sums, and the second of which is bounding these sums using the large sieve. The bulk of the work lies in the first step, and we have divided it into the case of totally real fields, carried out in section 5, and the modifications which are needed in the presence of complex places which are described in section 6. The application of the large sieve is carried out in section 7 .

\section{Sieving For mass equidistribution: The totally Real Case}

In this section we prove Proposition 9 below, which reduces the problem of bounding $\left\langle\phi F_{k}, F_{k}\right\rangle$ to one of bounding shifted convolution sums. We shall assume for simplicity in this section that $F$ is totally real, so that the key modifications in the unfolding argument can be seen more clearly, and leave the treatment of complex places for section [6. We will work with holomorphic forms rather than vector-valued ones, and so let $f$ be an $L^{2}$ normalised holomorphic Hecke eigenform of weight $k$ with associated automorphic representation $\pi$. We continue to assume that there exists $\nu>0$ such that $k_{i} \geq\|k\|^{\nu}$ for all $i$.

Proposition 9. Let $T \geq 1$ and $\epsilon>0$. Fix $h \in C_{0}^{\infty}\left(\mathbb{R}^{+}\right)$positive and let $g \in$ $C_{0}^{\infty}\left(\mathbb{F}^{+}\right)$be its n-fold product, and define $C_{g}=\langle E(g \mid z), 1\rangle / \operatorname{Vol}(Y)$. Fix an automorphic form $\phi$ with Fourier expansion

$$
\phi(z)=\sum_{\xi \in \mathcal{O}} a_{\xi}(y) e(\operatorname{tr}(\xi \kappa x)) .
$$

If $\phi$ is a Hecke-Maass cusp form, then

$$
\left\langle\phi F_{k}, F_{k}\right\rangle=C_{g}^{-1} T^{-n} \sum_{0<\|\xi\|<T^{1+\epsilon}} S_{\xi}(T)+O\left(T^{-n / 2}\right) .
$$

If $\phi$ is a pure incomplete Eisenstein series, then

$$
\left\langle\phi F_{k}, F_{k}\right\rangle=\frac{1}{\operatorname{Vol}(Y)}\langle\phi, 1\rangle+C_{g}^{-1} T^{-n} \sum_{0<\|\xi\|<T^{1+\epsilon}} S_{\xi}(T)+O\left(\frac{1+R_{k}(\pi)}{T^{n / 2}}\right)
$$

with

$$
R_{k}(\pi)=\frac{1}{\sqrt{N k} L\left(1, \operatorname{sym}^{2} \pi\right)} \sum_{m} \int_{-\infty}^{+\infty} \frac{\left|L\left(1 / 2+i t, \operatorname{sym}^{2} \pi \otimes \lambda_{-m}\right)\right|}{(|t|+\|m\|+1)^{A}} d t .
$$


Furthermore, we have the bound

$$
\begin{aligned}
S_{\xi}(T) \ll \frac{\left|a_{\xi}\left(T^{-1}\right)\right|}{N k L\left(1, \mathrm{sym}^{2} \pi\right)}\left(\sum_{\eta>0}\left|\lambda_{\pi}(\eta) \lambda_{\pi}(\eta+\xi)\right|\right. & \prod_{i=1}^{n} h\left(\frac{T\left(k_{i}-1\right)}{4 \pi\left(\eta_{i}+\xi_{i} / 2\right)}\right) \\
& \left.+O\left(N k\|k\|^{-\nu+\epsilon} T^{n+\epsilon}\right)\right) .
\end{aligned}
$$

The bound we shall apply to the shifted convolution sums appearing in Proposition 9 is given below; it will be proven in section 7 following Holowinsky, although it should be noted that this result may also be derived from the works of Nair 27. and Nair-Tenenbaum [28].

Proposition 10. Let $\lambda_{1}$ and $\lambda_{2}$ be multiplicative functions on $\mathcal{O}^{+}$satisfying $\left|\lambda_{i}(\eta)\right| \leq \tau_{m}(\eta)$ for some $m$. For any $x=\left(x_{i}\right)$ sufficiently large with respect to $\epsilon$ and satisfying $x_{i} \geq\|x\|^{\nu}$, and any fixed $\xi \in \mathcal{O}$ satisfying $0<\|\xi\| \leq\|x\|^{\nu}$, we have

$$
\sum_{0<\eta<x}\left|\lambda_{1}(\eta) \lambda_{2}(\eta+\xi)\right| \ll \frac{\tau(\xi) N x}{(\log \|x\|)^{2-\epsilon}} \prod_{N \mathfrak{p} \leq z}\left(1+\frac{\left|\lambda_{1}(\mathfrak{p})\right|+\left|\lambda_{2}(\mathfrak{p})\right|}{N \mathfrak{p}}\right),
$$

where $z=\|x\|^{1 / s}$ and $s=\epsilon \log \log \|x\|$.

To deduce Theorem 4 in the totally real case from Propositions 9 and 10 first apply Proposition 10 with $\lambda_{1}=\lambda_{2}=\lambda_{\pi}$ and $x=T k$. Because we shall choose $T$ to be bounded by any positive power of $\|k\|$, we see that $z=o(\|k\|)$ so that we have (19)

$$
\sum_{\eta>0}\left|\lambda_{\pi}(\eta) \lambda_{\pi}(\eta+\xi)\right| \prod_{i=1}^{n} h\left(\frac{T\left(k_{i}-1\right)}{4 \pi\left(\eta_{i}+\xi_{i} / 2\right)}\right) \ll \frac{\tau(\xi) T^{n} N k}{(\log \|k\|)^{2-\epsilon}} \prod_{N \mathfrak{p} \leq\|k\|}\left(1+\frac{2\left|\lambda_{\pi}(\mathfrak{p})\right|}{N \mathfrak{p}}\right) .
$$

In the case of $\phi$ a Maass form, we substitute this into (18) and bound $\left|a_{\xi}\left(T^{-1}\right)\right|$ by $|\rho(\xi)| T^{-n / 2+\epsilon}$ using Lemma 11 from section 5.1 below $(\rho(\xi)$ denotes the Hecke eigenvalue of $\phi$ ). As $T$ is bounded above by any positive power of $\|k\|$, (18) then becomes

$$
S_{\xi}(T) \ll \frac{|\rho(\xi)| \tau(\xi) T^{n / 2+\epsilon}}{L\left(1, \operatorname{sym}^{2} \pi\right)(\log \|k\|)^{2-\epsilon}} \prod_{N \mathfrak{p} \leq\|k\|}\left(1+\frac{2\left|\lambda_{\pi}(\mathfrak{p})\right|}{N \mathfrak{p}}\right)+O\left(\|k\|^{-\nu+\epsilon}\right) .
$$

Substituting this into (15) and applying the Ramanujan bound on average (20), we obtain

$$
\left\langle\phi F_{k}, F_{k}\right\rangle \ll \frac{T^{n / 2}(T \log \|k\|)^{\epsilon}}{(\log \|k\|)^{2} L\left(1, \operatorname{sym}^{2} \pi\right)} \prod_{N \mathfrak{p} \leq\|k\|}\left(1+\frac{2\left|\lambda_{\pi}(\mathfrak{p})\right|}{N \mathfrak{p}}\right)+O\left(T^{-n / 2}\right)
$$

which gives (6) on choosing $T^{n}=M_{k}(\pi)^{-1}$. The derivation of (7) in the pure incomplete Eisenstein series case is similar.

The organisation of this section is as follows. In section 5.1 we prove some results we shall need on the Fourier coefficients of $\phi$ and $f$. In section 5.2 we introduce the regularised unfolding integral which lies at the heart of our proof, before using it to relate $\left\langle\phi F_{k}, F_{k}\right\rangle$ to shifted convolution sums in section 5.3. 
5.1. Fourier coefficient calculations. In this section we present some bounds and normalisations we shall need for the Fourier coefficients of $\phi$ and $f$. If $\phi$ is an automorphic form on $\Gamma \backslash \mathbb{H}^{n}$, we may expand it in a Fourier series as

$$
\phi(z)=a_{0}(y)+\sum_{\xi \neq 0} a_{\xi}(y) e(\operatorname{tr}(\xi \kappa x))
$$

with $a_{0}(y)=0$ if $\phi$ is a cusp form. If $\phi$ is a fixed Maass cusp form with spectral parameter $t=\left(t_{i}\right)$, then we have the expansion

$$
\phi(z)=\sqrt{N y} \sum_{\xi \neq 0} \rho(\xi) \prod_{p=1}^{n} K_{i t_{p}}\left(2 \pi\left|\xi_{p}\right| \kappa_{p} y_{p}\right) e(\operatorname{tr}(\xi \kappa x)),
$$

where the Hecke eigenvalues $\rho(\xi)$ satisfy the Ramanujan bound on average, i.e.

$$
\sum_{\|\xi\| \leq T}|\rho(\xi)| \ll T^{n}
$$

If $\phi$ is a pure incomplete Eisenstein series $E(\psi, m \mid z)$, we may determine its Fourier coefficients in terms of the coefficients of the complete Eisenstein series $E(s, m, z)$. The Fourier expansion of these series was calculated by Efrat [5] to be

$$
\begin{gathered}
E(s, m, z)=N y^{s} \lambda_{m}(y)+\phi(s, m) N y^{1-s} \lambda_{-m}(y)+\frac{2^{n} \pi^{n s}}{\sqrt{D}} N y^{1 / 2} \times \\
\sum_{\xi \neq 0} N(\xi \kappa)^{s-1 / 2} \lambda_{m}(\xi \kappa) \prod_{p=1}^{n} \frac{K_{s+\beta(m, p)-1 / 2}\left(2 \pi\left|\xi_{p}\right| \kappa_{p} y_{p}\right)}{\Gamma(s+\beta(m, p))} \frac{\sigma_{1-2 s,-2 m}(\xi \kappa)}{\zeta\left(2 s, \lambda_{-2 m}\right)} e(\operatorname{tr}(\xi \kappa x)),
\end{gathered}
$$

where $\beta(m, p)$ is as in (1) and

$$
\begin{aligned}
\phi(s) & =\frac{\pi^{n / 2}}{\sqrt{D}} \prod_{p=1}^{n} \frac{\Gamma(s+\beta(m, p)-1 / 2)}{\Gamma(s+\beta(m, p))} \frac{\zeta\left(2 s-1, \lambda_{-2 m}\right)}{\zeta\left(2 s, \lambda_{-2 m}\right)}=\frac{\theta(s-1 / 2)}{\theta(s)}, \\
\theta(s) & =\pi^{-n s} D^{s} \prod_{p=1}^{n} \Gamma(s+\beta(m, p)) \zeta\left(2 s, \lambda_{-2 m}\right), \\
\sigma_{1-2 s,-2 m}(\xi \kappa) & =\sum_{\substack{(c) \\
\xi / c \in \mathcal{O}}} \frac{\lambda_{-2 m}(c)}{|N c|^{2 s-1}} .
\end{aligned}
$$

We have

$$
E(\psi, m \mid z)=\frac{1}{2 \pi i} \int_{(2)} \Psi(-s) E(s, m, z) d s,
$$

where $\Psi$ is the Mellin transform of $\psi$, and we may use this to calculate the Fourier coefficients of $E(\psi, m \mid z)$. In the case of the constant term, we have

$$
\begin{aligned}
a_{0}(y) & =\frac{1}{2 \pi i} \int_{(2)} \Psi(-s)\left(N y^{s} \lambda_{m}(y)+\phi(s, m) N y^{1-s} \lambda_{-m}(y)\right) d s \\
& =\psi(N y) \lambda_{m}(y)+O\left(N y^{-1}\right) .
\end{aligned}
$$

Moving the line of integration to $\sigma=1 / 2$ we obtain

$$
a_{0}(y)=\frac{1}{\operatorname{Vol}(Y)}\langle E(\psi, m \mid z), 1\rangle+O\left(N y^{1 / 2}\right),
$$


where the main term is only nonzero for $m=0$. Doing the same for nonzero $\xi$ gives

$$
\begin{aligned}
a_{\xi}(y)=\frac{2^{n} \pi^{n / 2} N y^{1 / 2}}{2 \pi i \sqrt{D}} \int_{-\infty}^{\infty} \pi^{n i t} & \Psi(-1 / 2-i t) N(\xi \kappa)^{i t} \lambda_{m}(\xi \kappa) \\
& \times \prod_{p=1}^{n} \frac{K_{i t+\beta(m, p)}\left(2 \pi\left|\xi_{p}\right| \kappa_{p} y_{p}\right)}{\Gamma(1 / 2+i t+\beta(m, p))} \frac{\sigma_{-2 i t,-2 m}(\xi \kappa)}{\zeta\left(2 s, \lambda_{-2 m}\right)} d t .
\end{aligned}
$$

We may apply the bound

$$
K_{i r}(y) \ll|\Gamma(1 / 2+i r)|\left(\frac{1+|r|}{y}\right)^{A}\left(1+\frac{1+|r|}{y}\right)^{\epsilon},
$$

valid for any integer $A \geq 0$ and $\epsilon>0$, to this to obtain

$$
a_{\xi}(y) \ll \tau(\xi) N y^{1 / 2}\|\xi y\|^{-A} \prod_{i=1}^{n}\left(1+\frac{1}{\xi_{i} y_{i}}\right)^{\epsilon} .
$$

Similar bounds are valid for $\phi$ a Maass cusp form. The bounds for both varieties of the form are summarised in the following lemma:

Lemma 11. Let $\phi$ be an automorphic form on $Y$ with Fourier series expansion

$$
\phi(z)=a_{0}(y)+\sum_{\xi \neq 0} a_{\xi}(y) e(\operatorname{tr}(\xi \kappa x)) .
$$

If $\phi$ is a Maass cusp form, then $a_{0}(y)=0$ and for $\xi \neq 0$ we have

$$
a_{\xi}(y) \ll|\rho(\xi)| N y^{1 / 2}\|\xi y\|^{-A} \prod_{i=1}^{n}\left(1+\frac{1}{\xi_{i} y_{i}}\right)^{\epsilon}
$$

for any integer $A \geq 0$ and any $\epsilon>0$. If $\phi$ is a pure incomplete Eisenstein series, then

and for $\xi \neq 0$ we have

$$
a_{0}(y)=\frac{1}{\operatorname{Vol}(Y)}\langle\phi, 1\rangle+O\left(N y^{1 / 2}\right)
$$

$$
a_{\xi}(y) \ll \tau(\xi) N y^{1 / 2}\|\xi y\|^{-A} \prod_{i=1}^{n}\left(1+\frac{1}{\xi_{i} y_{i}}\right)^{\epsilon}
$$

for any integer $A \geq 0$ and any $\epsilon>0$.

As we shall work with the Fourier expansion of $f$ rather than that of $F_{k}$, the $L^{2}$ normalisation of $a_{f}(1)$ differs slightly from the one given in section 2.4. We write $f$ as

$$
f(z)=\sum_{\eta>0} a_{f}(\eta) e(\operatorname{tr}(\eta \kappa z))
$$

where the coefficients $a_{f}(\eta)$ continue to satisfy (4), and the bound $\left|\lambda_{\pi}(\xi)\right| \leq \tau(\xi)$ is known by the work of Blasius [3] and Deligne. The correct normalisation of $a_{f}(1)$ so that $\left\langle F_{k}, F_{k}\right\rangle=1$ is

$$
\left|a_{f}(1)\right|^{2}=\frac{\pi^{n} / 2}{D L\left(1, \operatorname{sym}^{2} \pi\right)} \kappa^{k} \prod_{i=1}^{n} \frac{(4 \pi)^{k_{i}}}{\Gamma\left(k_{i}\right)} .
$$


5.2. The regularised unfolding integral $I_{\phi}(T)$. In this section, we introduce the integral $I_{\phi}(T)$. Proposition 9 will follow from comparing two different asymptotic formulae for this integral, the first of which has the main term $T^{n}\left\langle\phi F_{k}, F_{k}\right\rangle$, and the second of which is expressed in terms of shifted convolution sums. Choose a positive function $h \in C_{0}^{\infty}\left(\mathbb{R}^{+}\right)$, and let $g \in C_{0}^{\infty}\left(\mathbb{F}^{+}\right)$be its $n$-fold product. Let

$$
\widetilde{g}=\sum_{u \in \mathcal{O}_{+}^{\times}} g(u y)
$$

be the symmetrisation of $g$ under the action of $\mathcal{O}_{+}^{\times}$, and let

$$
G(s, m)=\int_{\mathbb{F}^{+}} g(y) N y^{s-1} \lambda_{m}(y) d y
$$

be the Mellin transform of $\widetilde{g}$ thought of as a function on $\mathbb{F}^{+} / \mathcal{O}_{+}^{\times}$. If $\mathbb{F}_{+}^{1}$ denotes the multiplicative subgroup of norm 1 elements of $\mathbb{F}^{\times}$, we may use the formula of Efrat [5] for the volume of $\mathbb{F}_{+}^{1} / \mathcal{O}_{+}^{\times}$to invert this, obtaining

$$
\widetilde{g}(y)=\frac{1}{2^{n} \pi i R} \sum_{m} \int_{(\sigma)} G(-s,-m) N y^{s} \lambda_{m}(y) d s .
$$

Let $T \geq 1$, and define $I_{\phi}(T)$ to be the integral

$$
I_{\phi}(T)=\int_{\mathbb{F}^{+}} g(T y) N y^{-2}\left(\int_{\mathbb{F} / \mathcal{O}} \phi(z)\left|F_{k}(z)\right|^{2} d x\right) d y .
$$

By symmetrising under the action of $\mathcal{O}_{+}^{\times}$on $\mathbb{F}^{+}$, substituting (24) and refolding the Eisenstein series, we see that $I_{\phi}(T)$ may also be written in the form

$$
I_{\phi}(T)=\frac{1}{2^{n} \pi i R} \sum_{m} \int_{(\sigma)} G(-s,-m) T^{n s} \int_{Y} E(s, m, z) \phi(z)\left|F_{k}(z)\right|^{2} d v d s .
$$

The following lemma relates $I_{\phi}(T)$ to the inner product $\left\langle\phi F_{k}, F_{k}\right\rangle$.

Lemma 12. For $\phi$ a fixed Hecke-Maass cusp form or pure incomplete Eisenstein series we have

$$
I_{\phi}(T)=C_{g}\left\langle\phi F_{k}, F_{k}\right\rangle T^{n}+O\left(T^{n / 2}\right),
$$

where $C_{g}$ is as in Proposition 9 ,

Proof. Starting with equation (26) and moving the contour of integration to the line $\operatorname{Re}(s)=1 / 2$, we write

$$
I_{\phi}(T)=C_{g}\left\langle\phi F_{k}, F_{k}\right\rangle T^{n}+R_{\phi}(T)
$$

with $C_{g}$ coming from the pole of the Eisenstein series at $s=1$ (see section 11.3 for this calculation). $R_{\phi}(T)$ is the remaining integral along $\operatorname{Re}(s)=1 / 2$,

$$
R_{\phi}(T)=\int_{Y} p(z) \phi(z)\left|F_{k}(z)\right|^{2} d v
$$

with

$$
p(z)=\frac{1}{2^{n} \pi i R} \sum_{m} \int_{(1 / 2)} G(-s,-m) T^{n s} E(s, m, z) d s .
$$

The Fourier series expansion of $E(s, m, z)$ and the bound (21) for $K_{i r}$ give

$$
E(s, m, z) \ll N y^{1 / 2}+N y^{-n-1 / 2}(|s|+\|m\|)^{n+2}\left(1+(|s|+\|m\|) N y^{-1 / n}\right)^{\epsilon},
$$


so that $p(z) \ll \sqrt{N y} T^{n / 2}$ if $N y \gg 1$. It follows from this and the rapid decay of $\phi(z)\left|F_{k}(z)\right|^{2}$ that $R_{\phi}(T) \ll_{\phi, g} T^{n / 2}$.

Substituting the formula (27) for $I_{\phi}(T)$ into (25) gives

$$
C_{g}\left\langle\phi F_{k}, F_{k}\right\rangle T^{n}+O\left(T^{n / 2}\right)=\int_{\mathbb{F}^{+}} g(T y) N y^{-2}\left(\int_{\mathbb{F} / \mathcal{O}} \phi(z)\left|F_{k}(z)\right|^{2} d x\right) d y,
$$

and we shall express the RHS of this in terms of shifted convolution sums after truncating our fixed form $\phi$. Recall that this had a Fourier expansion

$$
\phi(z)=\sum_{\xi \in \mathcal{O}} a_{\xi}(y) e(\operatorname{tr}(\xi \kappa x))
$$

with the $a_{\xi}(y)$ bounded as in Lemma 11, If $\phi$ is a pure incomplete Eisenstein series, then we find that the contribution to $I_{\phi}(T)$ from the tail of (29) with $\|\xi\| \geq T^{1+\epsilon}$ for any $\epsilon>0$ is bounded by

$$
I_{1}(T) T^{-n / 2+A+\epsilon^{\prime}} \sum_{\|\xi\| \geq T^{1+\epsilon}} \tau(\xi)\|\xi\|^{-A+\epsilon^{\prime}} \ll T^{3 n / 2+\epsilon(n+1-A)}
$$

by the support of $g$ and Lemma 11 (which is the source of the $\epsilon^{\prime}$ ). Here $I_{1}(T)$ is equal to $I_{\phi}(T)$ with $\phi$ chosen to be the constant function. It follows from this that the contribution of these terms to $I_{\phi}(T)$ is $\ll T^{n / 2}$ after choosing $A$ sufficiently large with respect to $\epsilon$, and a similar argument works when $\phi$ is a fixed cusp form. If we define $\phi^{*}$ to be the truncated function

$$
\phi^{*}(z)=\sum_{\|\xi\|<T^{1+\epsilon}} a_{\xi}(y) e(\operatorname{tr}(\xi \kappa x))
$$

we therefore have

$$
C_{g}\left\langle\phi F_{k}, F_{k}\right\rangle T^{n}=\int_{\mathbb{F}^{+}} g(T y) N y^{-2}\left(\int_{\mathbb{F} / \mathcal{O}} \phi^{*}(z)\left|F_{k}(z)\right|^{2} d x\right) d y+O\left(T^{n / 2}\right) .
$$

5.3. Extracting shifted convolution sums. In this section we shall expand the RHS of (30) using the Fourier expansion of $\phi^{*}$, writing

$$
C_{g}\left\langle\phi F_{k}, F_{k}\right\rangle T^{n}=S_{0}(T)+\sum_{0<\|\xi\|<T^{1+\epsilon}} S_{\xi}(T)+O\left(T^{n / 2}\right),
$$

where for any $\xi \in \mathcal{O}$ we define

$$
S_{\xi}(T)=\int_{\mathbb{F}^{+}} g(T y) N y^{-2}\left(\int_{\mathbb{F} / \mathcal{O}} a_{\xi}(y) e(\operatorname{tr}(\xi \kappa x))\left|F_{k}(z)\right|^{2} d x\right) d y .
$$

Note that (15) of Proposition 9 follows from this definition. The aim of this section is to analyse the objects $S_{\xi}(T)$ so that when we divide through by $C_{g} T^{n}$ we have the remaining equations and bounds of Proposition 9 .

We first note that $S_{0}(T)=0$ when $\phi$ is a cusp form, and by Lemma 11 we have

$$
S_{0}(T)=\left(\frac{\langle\phi, 1\rangle}{\operatorname{Vol}(Y)}+O\left(T^{-n / 2}\right)\right) I_{1}(T)
$$


when $\phi$ is a pure incomplete Eisenstein series. We shall treat the two cases $I_{1}(T)$ and $S_{\xi}(T)$ for $\xi \neq 0$ seperately, beginning with the latter. Squaring out $\left|F_{k}(z)\right|^{2}$ and integrating in $x$ gives

$$
S_{\xi}(T)=\sqrt{D} \sum_{\eta>0} \overline{a_{f}(\eta)} a_{f}(\eta+\xi)\left(\int_{\mathbb{F}^{+}} g(T y) a_{\xi}(y) y^{k-2} e^{-2 \pi \operatorname{tr}((2 \eta+\xi) \kappa y)} d y\right) .
$$

As the exponentials and $g$ are positive, this satisfies

$$
S_{\xi}(T) \ll\left|a_{\xi}\left(T^{-1}\right)\right| \sum_{\eta>0}\left|a_{f}(\eta) a_{f}(\eta+\xi)\right|\left(\int_{\mathbb{F}^{+}} g(T y) y^{k-2} e^{-2 \pi \operatorname{tr}((2 \eta+\xi) \kappa y)} d y\right) .
$$

Appealing to the Mellin transform $H$ of $h$ and applying the normalisations (44) and (22) of $a_{f}(\eta)$ and $a_{f}(\eta+\xi)$, we may integrate in $y$ to obtain

$$
\begin{aligned}
& \text { (33) } \quad S_{\xi}(T) \ll \frac{\left|a_{\xi}\left(T^{-1}\right)\right|}{N k L\left(1, \mathrm{sym}^{2} \pi\right)} \sum_{\eta>0}\left|\lambda_{\pi}(\eta) \lambda_{\pi}(\eta+\xi)\right| \\
& \times \prod_{i=1}^{n}\left(\frac{\sqrt{\eta_{i}\left(\eta_{i}+\xi_{i}\right)}}{\eta_{i}+\xi_{i} / 2}\right)^{k_{i}-1} \frac{1}{2 \pi i} \int_{(\sigma)} H(-s)\left(\frac{T}{4 \pi \kappa_{i}\left(\eta_{i}+\xi_{i} / 2\right)}\right)^{s} \frac{\Gamma\left(s+k_{i}-1\right)}{\Gamma\left(k_{i}-1\right)} d s .
\end{aligned}
$$

Note that $\sqrt{\eta_{i}\left(\eta_{i}+\xi_{i}\right)} \leq \eta_{i}+\xi_{i} / 2$, so that these factors may be omitted.

We may simplify this expression using a lemma seen in the work of Luo and Sarnak 23]. Using Stirling's formula, they prove the asymptotic

$$
\frac{\Gamma\left(s+k_{i}-1\right)}{\Gamma\left(k_{i}-1\right)}=\left(k_{i}-1\right)^{s}\left(1+O_{a, b}\left((|s|+1)^{2} k_{i}^{-1}\right)\right)
$$

in any vertical strip $0<a \leq \operatorname{Re}(s) \leq b$. If we apply this to (33) we may invert the Mellin transform of $h$ to obtain

$$
\begin{aligned}
& S_{\xi}(T) \ll \frac{\left|a_{\xi}\left(T^{-1}\right)\right|}{N k L\left(1, \operatorname{sym}^{2} \pi\right)} \sum_{\eta>0}\left|\lambda_{\pi}(\eta) \lambda_{\pi}(\eta+\xi)\right| \\
& \times \prod_{i=1}^{n}\left(h\left(\frac{T\left(k_{i}-1\right)}{4 \pi\left(\eta_{i}+\xi_{i} / 2\right)}\right)+O\left(k_{i}^{\epsilon}\left(\frac{T}{\eta_{i}+\xi_{i} / 2}\right)^{1+\epsilon}\right)\right) .
\end{aligned}
$$

The final step in proving (18) from this is showing that when the product in (35) is expanded out, the total contribution from all the error terms is « $N k\|k\|^{-\nu+\epsilon} T^{n+\epsilon}$. It is enough to consider one such term which contains 'main term' factors at the first $t$ places and error term factors at the last $n-t$. As the factors of $h$ truncate the sum at the first $t$ places, the contribution from this term is bounded by

$$
\ll \sum_{\substack{\eta \in \mathcal{O} \\\left|\eta_{i}+\xi_{i} / 2\right| \ll T k_{i}, i \leq t}}\left|\lambda_{\pi}(\eta) \lambda_{\pi}(\eta+\xi)\right| \prod_{i>t} k_{i}^{\epsilon}\left(\frac{T}{\eta_{i}+\xi_{i} / 2}\right)^{1+\epsilon} .
$$

If we let $\tau=\eta+\xi / 2$, then $\tau \in \frac{1}{2} \mathcal{O}^{+}$(because we may assume $\eta_{i}$ and $\eta_{i}+\xi_{i}$ are positive), and because $\left|\xi_{i}\right| \ll T^{1+\epsilon}$ we have $\tau_{i}+T^{2} \gg \max \left(\eta_{i}, \eta_{i}+\xi_{i}\right)$ for all $i$. Therefore by Deligne's bound,

$$
\lambda_{\pi}(\eta) \lambda_{\pi}(\eta+\xi) \ll N \eta^{\epsilon} N(\eta+\xi)^{\epsilon} \ll\|\tau\|^{\epsilon}+T^{\epsilon},
$$


and the expression (36) may be simplified to

$$
\ll \sum_{\tau_{i} \ll T k_{i}, i \leq t}\left(\|\tau\|^{\epsilon}+T^{\epsilon}\right) \prod_{i>t} k_{i}^{\epsilon} T^{1+\epsilon} \tau_{i}^{-1-\epsilon} .
$$

Because $\tau_{i} \ll T k_{i}$ for $i \leq t$, this may be further reduced to

$$
\ll N k^{\epsilon} T^{n-t+\epsilon} \sum_{\substack{\tau_{i} \ll T k_{i} \\ i \leq t}} \prod_{i>t} \tau_{i}^{-1-\epsilon} .
$$

If we project the set $\left\{\tau \in \frac{1}{2} \mathcal{O}^{+}: \tau_{i} \ll T k_{i}\right.$ for $\left.i \leq t\right\}$ onto the last $n-t$ real places, we obtain a set $\mathcal{O}^{\prime} \in \mathbb{R}^{n-t}$, any two of whose elements are at a distance $\gg \delta=\left(T^{t} \prod_{i \leq t} k_{i}\right)^{-1 /(n-t)}$ from each other and the origin. The sum above may therefore be bounded by

$$
\begin{aligned}
& \ll T^{t} \prod_{i \leq t} k_{i} \int_{x_{i} \gg \delta} \prod_{i>t} x_{i}^{-1-\epsilon} d x_{i} \\
& \ll T^{t+\epsilon} \prod_{i \leq t} k_{i}^{1+\epsilon},
\end{aligned}
$$

so that the total contribution of our error term is $\ll N k^{\epsilon} T^{n+\epsilon} \prod_{i<t} k_{i}^{1+\epsilon}$. As $t<n$, we are omitting a factor of size at least $\|k\|^{\nu}$ from $N k$, so this is $\ll N k\|k\|^{-\nu+\epsilon} T^{n+\epsilon}$, as required. Therefore

$$
\begin{array}{r}
S_{\xi}(T) \ll \frac{1}{N k L\left(1, \operatorname{sym}^{2} \pi\right)}\left|a_{\xi}\left(T^{-1}\right)\right|\left(\sum_{\eta>0}\left|\lambda_{\pi}(\eta) \lambda_{\pi}(\eta+\xi)\right| \prod_{i=1}^{n} h\left(\frac{T\left(k_{i}-1\right)}{4 \pi\left(\eta_{i}+\xi_{i} / 2\right)}\right)\right. \\
\left.+O\left(N k|k|^{-\nu+\epsilon} T^{n+\epsilon}\right)\right)
\end{array}
$$

which is the bound (18).

We now deal with the case $\xi=0$. Squaring out $\left|F_{k}(z)\right|^{2}$ and integrating in $x$ gives

$$
I_{1}(T)=\sqrt{D} \sum_{\eta>0}\left|a_{f}(\eta)\right|^{2}\left(\int_{\mathbb{F}^{+}} g(T y) y^{k-2} e^{-4 \pi \operatorname{tr}(\eta \kappa y)} d y\right) .
$$

Expressing $a_{f}$ in terms of $\lambda_{\pi}$ and symmetrising by the action of $\mathcal{O}_{+}^{\times}$, this becomes

$$
\begin{aligned}
I_{1}(T) & =\sqrt{D}\left|a_{f}(1)\right|^{2} \kappa^{1-k} \sum_{\eta>0}\left|\lambda_{\pi}(\eta)\right|^{2}\left(\int_{\mathbb{F}^{+}} g(T y)(\eta \kappa y)^{k-1} e^{-4 \pi \operatorname{tr}(\eta \kappa y)} d y^{\times}\right) \\
& =\sqrt{D}\left|a_{f}(1)\right|^{2} \kappa^{1-k} \sum_{(\eta)>0}\left|\lambda_{\pi}(\eta)\right|^{2}\left(\int_{\mathbb{F}^{+}} g(T y) \widetilde{\psi}(\eta \kappa y) d y^{\times}\right) \\
& =\sqrt{D}\left|a_{f}(1)\right|^{2} \kappa^{1-k} \sum_{(\eta)>0}\left|\lambda_{\pi}(\eta)\right|^{2}\left(\int_{\mathbb{F}^{+} / \mathcal{O}_{+}^{\times}} \widetilde{g}(T y) \widetilde{\psi}(\eta \kappa y) d y^{\times}\right),
\end{aligned}
$$

where

$$
\widetilde{g}(y)=\sum_{u \in \mathcal{O}_{+}^{\times}} g(u y), \quad \widetilde{\psi}(y)=\sum_{u \in \mathcal{O}_{+}^{\times}}(u y)^{k-1} \exp (-4 \pi \operatorname{tr}(u y)) .
$$


If we let $G(s, m)$ be the Mellin transform of $\widetilde{g}$ as in (23), then by the Mellin inversion formula we have

$$
\begin{aligned}
I_{1}(T)=\frac{\sqrt{D}}{2^{n-1} R}\left|a_{f}(1)\right|^{2} \kappa^{1-k} \sum_{(\eta)}\left|\lambda_{\pi}(\eta)\right|^{2} \frac{1}{2 \pi i} \sum_{m} \int_{(\sigma)} G(-s,-m)\left(\frac{T}{4 \pi}\right)^{n s} \\
\quad \times N(\eta \kappa)^{-s} \lambda_{-m}(\eta \kappa) \prod_{i=1}^{n}(4 \pi)^{-k_{i}+1} \Gamma\left(s+\beta(m, i)+k_{i}-1\right) d s .
\end{aligned}
$$

Forming the $L$-function from the sum over $\eta$, this becomes

$$
\begin{aligned}
I_{1}(T) & =\frac{\sqrt{D}}{2^{n-1} R}\left|a_{f}(1)\right|^{2} \kappa^{1-k-s} \lambda_{-m}(\kappa) \frac{1}{2 \pi i} \sum_{m} \int_{(\sigma)} G(-s,-m)\left(\frac{T}{4 \pi}\right)^{n s} \\
& \times L\left(s, \operatorname{sym}^{2} \pi \otimes \lambda_{-m}\right) \frac{L\left(s, \lambda_{-m}\right)}{L\left(2 s, \lambda_{-2 m}\right)} \prod_{i=1}^{n}(4 \pi)^{-k_{i}+1} \Gamma\left(s+\beta(m, i)+k_{i}-1\right) d s .
\end{aligned}
$$

When we substitute the value of $\left|a_{f}(1)\right|^{2}$ given by (22) and shift the line of integration to $\sigma=1 / 2$, we pick up a main term from the pole at $s=1$, which is

$$
\frac{\pi^{n} G(-1,0) T^{n}}{2 D \zeta_{F}(2)}
$$

and in section 11.3 it is shown that this agrees with the expected main term $C_{g} T^{n}$. We therefore have

$$
I_{1}(T)=C_{g} T^{n}+E_{1 / 2}(T)
$$

with

$$
\begin{aligned}
E_{1 / 2}(T)= & \frac{\left(2 \pi^{2}\right)^{n} N \kappa^{1 / 2} \lambda_{-m}(\kappa)}{R \sqrt{D} L\left(1, \operatorname{sym}^{2} \pi\right)} \frac{1}{2 \pi i} \sum_{m} \int_{(1 / 2)} G(-s,-m)\left(\frac{T}{4 \pi}\right)^{n s} \\
& \times L\left(s, \operatorname{sym}^{2} \pi \otimes \lambda_{-m}\right) \frac{L\left(s, \lambda_{-m}\right)}{L\left(2 s, \lambda_{-2 m}\right)} \prod_{i=1}^{n} \frac{\Gamma\left(s+\beta(m, i)+k_{i}-1\right)}{\left(k_{i}-1\right) \Gamma\left(k_{i}-1\right)} d s .
\end{aligned}
$$

If we apply the Luo-Sarnak lemma (34) to (38) in the form

$$
\left|\frac{\Gamma\left(s+\beta(m, i)+k_{i}-1\right)}{\Gamma\left(k_{i}-1\right)}\right| \ll\left(k_{i}-1\right)^{\operatorname{Re}(s)}(1+|s|+\|m\|)^{2},
$$

together with the rapid decay of $G(s, m)$, the convex bound for $L\left(\lambda_{-m}, s\right)$ and any lower bound of the form $L\left(\lambda_{-2 m}, 1+i t\right) \gg(|t|+\|m\|+1)^{-A}$, we obtain

$$
E_{1 / 2}(T) \ll\left(\frac{T^{n}}{N k}\right)^{1 / 2} \frac{1}{L\left(1, \mathrm{sym}^{2} \pi\right)} \sum_{m} \int_{-\infty}^{+\infty} \frac{\left|L\left(1 / 2+i t, \mathrm{sym}^{2} \pi \otimes \lambda_{-m}\right)\right|}{(|t|+\|m\|+1)^{A}} d t .
$$

The asymptotic (16) and bound (17) for the error now follow by combining (39), (37) and (31), which completes the proof of Proposition 9

\section{Sieving for mass equidistribution: The mixed Case}

In this section we generalise Proposition 9 to allow complex places of $F$. As we may no longer talk about holomorphic forms we now let $F_{k}$ be a vector-valued cohomological form with associated automorphic representation $\pi$, and as before assume the existence of a $\nu>0$ such that $k_{i} \geq\|k\|^{\nu}$ for all $i$. Our bound for $\left\langle\phi F_{k}, F_{k}\right\rangle$ in terms of shifted convolution sums is as follows. 
Proposition 13. Let $T \geq 1$ and $\epsilon>0$. Fix $h \in C_{0}^{\infty}\left(\mathbb{R}^{+}\right)$positive, let $g \in C_{0}^{\infty}\left(\mathbb{R}_{+}^{r}\right)$ be its $r$-fold product, and define $C_{g}=\langle E(g \mid z), 1\rangle / \operatorname{Vol}(Y)$. Let $J$ be the set of $r_{2^{-}}$ tuples $J=\left\{\left(j_{r_{1}+1}, \ldots, j_{r}\right) \mid 0 \leq j_{i} \leq k_{i}\right\}$. Fix an automorphic form $\phi$ with Fourier expansion

$$
\phi(z)=\sum_{\xi \in \mathcal{O}} a_{\xi}(y) e(\operatorname{tr}(\xi \kappa x)) .
$$

If $\phi$ is a Hecke-Maass cusp form, then

$$
\left\langle\phi F_{k}, F_{k}\right\rangle=C_{g}^{-1} T^{-n} \sum_{0<\|\xi\|<T^{1+\epsilon}} S_{\xi}(T)+O\left(T^{-n / 2}\right) .
$$

If $\phi$ is a pure incomplete Eisenstein series, then

$$
\left\langle\phi F_{k}, F_{k}\right\rangle=\frac{1}{\operatorname{Vol}(Y)}\langle\phi, 1\rangle+C_{g}^{-1} T^{-n} \sum_{0<\|\xi\|<T^{1+\epsilon}} S_{\xi}(T)+O\left(\frac{1+R_{k}(\pi)}{T^{n / 2}}\right)
$$

with

$$
R_{k}(\pi)=\frac{1}{\sqrt{N k} L\left(1, \operatorname{sym}^{2} \pi\right)} \sum_{m} \int_{-\infty}^{+\infty} \frac{\left|L\left(1 / 2+i t, \operatorname{sym}^{2} \pi \otimes \lambda_{-m}\right)\right|}{(|t|+\|m\|+1)^{A}} d t .
$$

Furthermore, we have the bound

$$
S_{\xi}(T) \ll \frac{\left|a_{\xi}\left(T^{-1}\right)\right|}{N k L\left(1, \operatorname{sym}^{2} \pi\right)}\left(\sum_{j \in J} A_{\xi, j}(T)+O\left(N k\|k\|^{-\nu+\epsilon} T^{n+\epsilon}\right)\right),
$$

where

$$
\begin{aligned}
A_{\xi, j}(T) \ll\left(\sum_{\eta>0}\left|\lambda_{\pi}(\eta) \lambda_{\pi}(\eta+\xi)\right|\right. & \prod_{i \leq r_{1}} \frac{1}{k_{i}} h\left(\frac{T\left(k_{i}-1\right)}{4 \pi\left|\eta_{i} \kappa_{i}\right|}\right) \\
\times & \left.\prod_{i>r_{1}} \frac{1}{k_{i}\left(k_{i}-j_{i}\right) j_{i}} h\left(\frac{T \sqrt{j_{i}\left(k_{i}-j_{i}\right)}}{2 \pi\left|\eta_{i} \kappa_{i}\right|}\right)\right) .
\end{aligned}
$$

Theorem 4 follows from combining this with Proposition 10 as in the totally real case. Most of the added difficulty in the proof of Proposition 13 comes from the changes to the Fourier expansion of $F_{k}$ in the presence of complex places. The first difference is that $F_{k}$ is vector-valued, which is the source of the summation over $J$ in (43). The second is that its Fourier coefficients contain Bessel functions, and so multiple Bessel integrals appear when we bound $S_{\xi}(T)$ in terms of shifted convolution sums. Section 6.1 below contains the Fourier expansions and $L^{2}$ normalisations of the automorphic forms on $Y$ with which we shall work, as well as the revised definition of $I_{\phi}(T)$ and its expression in terms of the shifted convolution integrals $S_{\xi}(T)$. The remainder of the proof of Proposition 13 will then lie in analysing $S_{\xi}(T)$, which we do in section 6.2 in the case $\xi \neq 0$ and in section 6.3 in the case $\xi=0$.

6.1. Revision of basic definitions. The bounds we shall use for the Fourier coefficients of Maass forms and Eisenstein series are essentially unchanged from the 
totally real case. If $\phi$ is a Maass cusp form with spectral parameter $t=\left(t_{i}\right)$, then we have the expansion

$$
\phi(z)=\sqrt{N y} \sum_{\xi \neq 0} \rho(\xi) \prod_{p=1}^{r} K_{i t_{p}}\left(2 \pi \delta_{p}\left|\xi_{p} \kappa_{p}\right| y_{p}\right) e(\operatorname{tr}(\xi \kappa x)),
$$

where the Hecke eigenvalues $\rho(\xi)$ continue to satisfy the bound (20).

The coefficients of a pure incomplete Eisenstein series may again be expressed in terms of those of the complete Eisenstein series $E(s, m, z)$. The Fourier expansion of these is computed in section 11.1. following Efrat in the totally real case [5], and is

$$
\begin{aligned}
E(s, m, z)=N y^{s} \lambda_{m}(y)+ & \phi(s, m) N y^{1-s} \lambda_{-m}(y)+\frac{2^{r} \pi^{n s-r_{2}}}{\sqrt{|D|}} \sqrt{N y} \\
& \times \sum_{\xi \neq 0} N(\delta \xi \kappa)^{s-1 / 2} \lambda_{m}(\delta \xi \kappa) \\
& \times \prod_{p=1}^{r} \frac{K_{\delta_{p}(s-1 / 2)+\beta(m, p)}\left(2 \pi \delta_{p}\left|\xi_{p} \kappa_{p}\right| y_{p}\right)}{\Gamma\left(\delta_{p} s+\beta(m, p)\right)} \frac{\sigma_{1-2 s,-2 m}(\xi \kappa)}{\zeta\left(2 s, \lambda_{-2 m}\right)} e(\operatorname{tr}(\xi \kappa x)),
\end{aligned}
$$

where $\beta(m, p)$ is as in (1) and

$$
\begin{aligned}
\phi(s)= & \frac{\pi^{n / 2}}{\sqrt{|D|}} \prod_{p \leq r_{1}} \frac{\Gamma(s+\beta(m, p)-1 / 2)}{\Gamma(s+\beta(m, p))} \\
& \times \prod_{p>r_{1}} \frac{2}{2 s+\beta(m, p)-1} \frac{\zeta\left(2 s-1, \lambda_{-2 m}\right)}{\zeta\left(2 s, \lambda_{-2 m}\right)} \\
= & \frac{\theta(s-1 / 2)}{\theta(s)}, \\
\theta(s)= & |D|^{s} \pi^{-n s} \prod_{p \leq r_{1}} \Gamma(s+\beta(m, p)) \\
& \times \prod_{p>r_{1}} 2^{-2 s} \Gamma(2 s+\beta(m, p)) \zeta\left(2 s, \lambda_{-2 m}\right), \\
\sigma_{1-2 s,-2 m}(\xi \kappa)= & \sum_{\substack{(c) \\
\xi / c \in \mathcal{O}}} \frac{\lambda_{-2 m}(c)}{|N c|^{2 s-1}} .
\end{aligned}
$$

By Mellin inversion we again have the two asymptotics

$$
\begin{aligned}
a_{0}(y) & =\psi(N y) \lambda_{m}(y)+O\left(N y^{-1}\right), \\
& =\frac{1}{\operatorname{Vol}(Y)}\langle E(\psi, m \mid z), 1\rangle+O\left(N y^{1 / 2}\right)
\end{aligned}
$$

for the zeroth Fourier coefficient of $E(\psi, m \mid z)$ as $N y$ tends to 0 and infinity. The bounds on the nonzero coefficients of Hecke-Maass cusp forms and pure incomplete Eisenstein series are also unchanged, and so Lemma 11 of section 5.1 continues to hold. We recall the formula for the Fourier expansion of the vector-valued function $F_{k}$ in $\mathbb{H}_{F}^{\prime}$ :

$$
F_{k}(z)=\sum_{\eta>0} a_{f}(\eta) \mathbf{K}_{k}(\eta \kappa y) e(\operatorname{tr}(\eta \kappa x))
$$


where $\mathbf{K}_{k}(y)$ are as in (2) and (3). As before, we assume the Ramanujan bound $\left|\lambda_{\pi}(\eta)\right| \leq \tau(\eta)$; see the discussion of section 3 for the circumstances under which this is known.

$I_{\phi}(T)$ is still a regularised unfolding integral over $\Gamma_{U}$, and when constructing it we bear in mind that $\mathbb{H}_{F} \simeq \mathbb{R}_{+}^{r} \times \mathbb{F}$, and $\Gamma_{U} \backslash \mathbb{H}_{F} \simeq \mathbb{R}_{+}^{r} \times(\mathbb{F} / \mathcal{O})$. To define $I_{\phi}(T)$ we therefore choose a positive function $h \in C_{0}^{\infty}\left(\mathbb{R}^{+}\right)$and let $g \in C_{0}^{\infty}\left(\mathbb{R}_{+}^{r}\right)$ be its $r$-fold product. Let

$$
\widetilde{g}=\sum_{u \in \mathcal{O}_{+}^{\times}} g(|u| y)
$$

be the symmetrisation of $g$ under the action of $\mathcal{O}_{+}^{\times}$, and let

$$
G(s, m)=\int_{\mathbb{R}_{+}^{r}} g(y) N y^{s} \lambda_{m}(y) d y^{\times}
$$

be the Mellin transform of $\widetilde{g}$ thought of as a function on $\mathbb{R}_{+}^{r} / \mathcal{O}_{+}^{\times}$. The expression (93) derived in section 11.3 for the volume form of $\mathbb{H}_{F}$ with respect to the natural coordinates in the cusp of $Y$ allows us to invert (23), giving

$$
\widetilde{g}(y)=\frac{1}{2 \pi i V_{c}} \sum_{m} \int_{(\sigma)} G(-s,-m) N y^{s} \lambda_{m}(y) d s .
$$

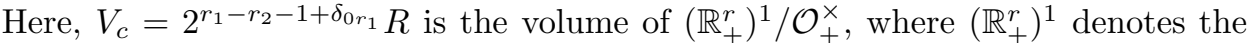
subgroup of norm 1 elements.

We now define $I_{\phi}(T)$ to be

$$
I_{\phi}(T)=\int_{\mathbb{R}_{+}^{r}} g(T y) N y^{-1}\left(\int_{\mathbb{F} / \mathcal{O}} \phi(z)\left|F_{k}(z)\right|^{2} d x\right) d y^{\times} .
$$

To rewrite this in terms of integrals against Eisenstein series, we symmetrise over $\mathcal{O}_{+}^{\times}$and substitute (47), giving

$$
\begin{aligned}
I_{\phi}(T)= & \frac{1}{2 \pi i V_{c}} \sum_{m} \int_{(\sigma)} G(-s,-m) T^{n s} \int_{\mathbb{R}_{+}^{r} / \mathcal{O}_{+}^{\times}} N y^{s-1} \lambda_{m}(y) \\
& \times \int_{\mathbb{F} / \mathcal{O}} \phi(z)\left|F_{k}(z)\right|^{2} d x d y^{\times} d s \\
= & \frac{\left|\mu_{+}\right|}{2 \pi i V_{c}} \sum_{m} \int_{(\sigma)} G(-s,-m) T^{n s} \int_{\Gamma_{\infty} \backslash \mathbb{H}_{F}} N y^{s} \lambda_{m}(y) \phi(z)\left|F_{k}(z)\right|^{2} d v d s \\
= & \frac{\left|\mu_{+}\right|}{2 \pi i V_{c}} \sum_{m} \int_{(\sigma)} G(-s,-m) T^{n s} \int_{Y} E(s, m, z) \phi(z)\left|F_{k}(z)\right|^{2} d v d s .
\end{aligned}
$$

On shifting the line of integration to $\sigma=1 / 2$, we have the asymptotic

$$
I_{\phi}(T)=C_{g}\left\langle\phi F_{k}, F_{k}\right\rangle T^{n}+O\left(T^{n / 2}\right) .
$$

(See section 11.3 for the verification that the residue at $s=1$ is correct.) Comparing this with the expression (48) for $I_{\phi}(T)$ and truncating the Fourier expansion of $\phi$ 
to those terms with $\|\xi\| \ll T^{1+\epsilon}$, we arrive at the equation

$$
\begin{aligned}
C_{g}\left\langle\phi F_{k}, F_{k}\right\rangle T^{n}+O\left(T^{n / 2}\right) & =\int_{\mathbb{R}_{+}^{r}} g(T y) N y^{-1}\left(\int_{\mathbb{F} / \mathcal{O}} \phi^{*}(z)\left|F_{k}(z)\right|^{2} d x\right) d y^{\times} \\
\text {where } \phi^{*}(z) & =\sum_{\|\xi\|<T^{1+\epsilon}} a_{\xi}(y) e(\operatorname{tr}(\xi \kappa x)) .
\end{aligned}
$$

This is the starting point for our analysis of Fourier coefficients.

6.2. Extracting shifted convolution sums: Nonzero shifts. Define $S_{\xi}(T)$ to be the contribution of the $\xi$ th Fourier coefficient of $\phi$ to (50) as before. It remains to estimate $S_{\xi}(T)$ in terms of shifted convolution sums, which we do first when $\xi \neq 0$. Squaring out $\left|F_{k}(z)\right|^{2}$ and integrating in $x$ gives

$$
\begin{aligned}
S_{\xi}(T)=2^{-r_{2}} & \sqrt{D} \sum_{\eta>0} a_{f}(\eta) \overline{a_{f}(\eta+\xi)} \\
& \times\left(\int_{\mathbb{R}_{+}^{r}} g(T y) a_{\xi}(y)\left\langle\mathbf{K}_{k}(\eta \kappa y), \mathbf{K}_{k}((\eta+\xi) \kappa y)\right\rangle N y^{-1} d y^{\times}\right) .
\end{aligned}
$$

Applying Hölder's inequality, this becomes

$$
\begin{aligned}
S_{\xi}(T) \ll & \left|a_{\xi}\left(T^{-1}\right)\right| \sum_{\eta>0}\left|a_{f}(\eta) a_{f}(\eta+\xi)\right|\left(\int _ { \mathbb { R } _ { + } ^ { r } } g ( T y ) \left[\left(\frac{N(\eta+\xi)}{N \eta}\right)^{1 / 2}\left|\mathbf{K}_{k}(\eta \kappa y)\right|^{2}\right.\right. \\
& \left.\left.+\left(\frac{N \eta}{N(\eta+\xi)}\right)^{1 / 2}\left|\mathbf{K}_{k}((\eta+\xi) \kappa y)\right|^{2}\right] N y^{-1} d y^{\times}\right) .
\end{aligned}
$$

The second term in this integral behaves identically to the first, and we ignore it for simplicity. Applying a change of variable and the normalisation (4) of $a_{f}(\eta)$, we have

$$
\begin{array}{r}
S_{\xi}(T) \ll\left|a_{\xi}\left(T^{-1}\right)\right| \sum_{\eta>0}\left|a_{f}(\eta) a_{f}(\eta+\xi)\right| N(\eta(\eta+\xi))^{1 / 2} \\
\times \int_{\mathbb{R}_{+}^{r}} g\left(T|\eta \kappa|^{-1} y\right)\left|\mathbf{K}_{k}(y)\right|^{2} N y^{-1} d y^{\times} \\
\ll\left|a_{\xi}\left(T^{-1}\right)\right|\left|a_{f}(1)\right|^{2} \sum_{\eta>0}\left|\lambda_{\pi}(\eta) \lambda_{\pi}(\eta+\xi)\right| \\
\times \int_{\mathbb{R}_{+}^{r}} g\left(T|\eta \kappa|^{-1} y\right)\left|\mathbf{K}_{k}(y)\right|^{2} N y^{-1} d y^{\times} .
\end{array}
$$

We have the following formula for $\left|K_{f}\right|^{2}$ from (2) and (3):

$$
\left|\mathbf{K}_{k}(y)\right|^{2}=\prod_{i \leq r_{1}} y_{i}^{k_{i}} \exp \left(-4 \pi y_{i}\right) \prod_{i>r_{1}} y_{i}^{k_{i}+2} \sum_{j=0}^{k_{i}}\left(\begin{array}{c}
k_{i} \\
j
\end{array}\right)\left|K_{k_{i} / 2-j}\left(4 \pi y_{i}\right)\right|^{2}
$$


where we now take $y_{i} \in \mathbb{R}$ for all $i$. For a multi-index $j=\left(j_{i}\right) \in J$ we define $K_{k, j}(y)$ to be the corresponding term in the formula for $\mathbf{K}_{k}(y)$, so that

$$
\left|K_{k, j}(y)\right|^{2}=\prod_{i \leq r_{1}} y_{i}^{k_{i}} \exp \left(-4 \pi y_{i}\right) \prod_{i>r_{1}} y_{i}^{k_{i}+2}\left(\begin{array}{c}
k_{i} \\
j_{i}
\end{array}\right)\left|K_{k_{i} / 2-j}\left(4 \pi y_{i}\right)\right|^{2},
$$

and we define $S_{\xi, j}(T)$ to be the corresponding term in $S_{\xi}(T)$.

We shall partition $J$ as $J_{0} \cup J_{1}$, where $J_{0}=\left\{j \mid \min \left(j_{i}, k_{i}-j_{i}\right)>k_{i}^{1 / 2}\right.$ for all $r_{1}<$ $i \leq r\}$. The reason for separating the indices in this way is that for $j \in J_{0}$, the arguments of all the Bessel functions appearing in (52) are bounded away from $\pm k_{i} / 2$. As a result, when we calculate the Mellin transforms of $\left|K_{k, j}(y)\right|^{2}$ the gamma factors which appear have arguments with large real parts, and so we may approximate them well using the Luo-Sarnak asymptotic (34). For $j \in J_{0}$ this lets us give good bounds for $S_{\xi, j}(T)$, while a weaker bound will suffice for the remaining terms because $J_{1}$ is small (in fact $\left|J_{1}\right| \ll\|k\|^{-\nu / 2}|J|$ ). We begin by deriving this weak bound for all $j$, interchanging the sum and integral in (51) to obtain

$S_{\xi, j}(T) \ll\left|a_{\xi}\left(T^{-1}\right)\right|\left|a_{f}(1)\right|^{2} \int_{\mathbb{R}_{+}^{r}}\left|K_{k, j}(y)\right|^{2} N y^{-1} \sum_{\eta>0}\left|\lambda_{\pi}(\eta) \lambda_{\pi}(\eta+\xi)\right| g\left(T|\eta \kappa|^{-1} y\right) d y^{\times}$.

The inner function

$$
\sum_{\eta>0}\left|\lambda_{\pi}(\eta) \lambda_{\pi}(\eta+\xi)\right| g\left(T|\eta \kappa|^{-1} y\right)
$$

is bounded above by the sum over $\eta$ such that $T y_{i} \ll\left|\eta_{i}\right| \ll T y_{i}$ for all $i$, weighted by $\left|\lambda_{\pi}(\eta) \lambda_{\pi}(\eta+\xi)\right| \ll\|T y\|^{\epsilon}$, from which it follows that

$$
\sum_{\eta>0}\left|\lambda_{\pi}(\eta) \lambda_{\pi}(\eta+\xi)\right| g\left(T|\eta \kappa|^{-1} y\right) \ll T^{n+\epsilon} N y \prod_{i=1}^{r}\left(1+y_{i}^{\epsilon}\right) .
$$

Applying this to (53) gives the upper bound

$$
S_{\xi, j}(T) \ll T^{n+\epsilon}\left|a_{\xi}\left(T^{-1}\right)\right|\left|a_{f}(1)\right|^{2} \int_{\mathbb{R}_{+}^{r}}\left|K_{k, j}(y)\right|^{2} \prod_{i=1}^{r}\left(1+y_{i}^{\epsilon}\right) d y^{\times} .
$$

The integral in (54) factorises as a product over the Archimedean places. At each place, we may bound the product of the local integral and the corresponding terms in the expression (5) for $\left|a_{f}(1)\right|^{2}$, before substituting these to give a bound for $S_{\xi, j}(T)$. The factor corresponding to a real place $i \leq r_{1}$ is

$$
\begin{aligned}
\frac{(4 \pi)^{k_{i}}}{\Gamma\left(k_{i}\right)} \int_{0}^{\infty} y^{k_{i}} \exp (-4 \pi y)\left(1+y^{\epsilon}\right) d y^{\times} & \ll \frac{1}{\Gamma\left(k_{i}\right)}\left(\Gamma\left(k_{i}\right)+\Gamma\left(k_{i}+\epsilon\right)\right) \\
& \ll k_{i}^{\epsilon} .
\end{aligned}
$$

For $i>r_{1}$, it is

$$
\frac{(2 \pi)^{k_{i}}}{\Gamma\left(k_{i} / 2+1\right)^{2}}\left(\begin{array}{c}
k_{i} \\
j_{i}
\end{array}\right) \int_{0}^{\infty} y^{k_{i}+2}\left|K_{k_{i} / 2-j_{i}}(4 \pi y)\right|^{2}\left(1+y^{\epsilon}\right) d y^{\times},
$$

and we may evaluate this using the following formula ([11, 6.576):

$$
\int_{0}^{\infty} y^{\lambda} K_{\mu}(y) K_{\nu}(y) d y=\frac{2^{\lambda-2}}{\Gamma(\lambda+1)} \prod_{ \pm} \Gamma\left(\frac{1+\lambda \pm \mu \pm \nu}{2}\right) .
$$


Applying this, we obtain

$$
\begin{aligned}
& \frac{1}{\Gamma\left(k_{i} / 2+1\right)^{2}}\left(\begin{array}{c}
k_{i} \\
j_{i}
\end{array}\right)\left(\frac{\Gamma\left(k_{i} / 2+1\right)^{2}}{\Gamma\left(k_{i}+2\right)} \Gamma\left(j_{i}+1\right) \Gamma\left(k_{i}-j_{i}+1\right)\right. \\
& \left.\quad+\frac{\Gamma\left(k_{i} / 2+1+\epsilon / 2\right)^{2}}{\Gamma\left(k_{i}+2+\epsilon\right)} \Gamma\left(j_{i}+1+\epsilon / 2\right) \Gamma\left(k_{i}-j_{i}+1+\epsilon / 2\right)\right) \ll k_{i}^{1-\epsilon} .
\end{aligned}
$$

Multiplying the bounds (55) and (57) and substituting the remaining terms in the expression (5) for $\left|a_{f}(1)\right|^{2}$ gives

$$
S_{\xi, j}(T) \ll \frac{\left|a_{\xi}\left(T^{-1}\right)\right| T^{n+\epsilon}}{L\left(1, \mathrm{sym}^{2} \pi\right)} N k^{\epsilon} \prod_{i>r_{1}} k_{i}^{-1}
$$

and so the contribution to $S_{\xi}(T)$ from all $j \in J_{1}$ is bounded above by

$$
\ll \frac{\left|a_{\xi}\left(T^{-1}\right)\right| T^{n+\epsilon}}{L\left(1, \operatorname{sym}^{2} \pi\right)}\|k\|^{-\nu / 2+\epsilon} .
$$

We shall treat the terms with $j \in J_{0}$ more carefully, by preserving the eigenvalue sum and analysing the weight provided by the integral. As before, we shall factorise the integral in (51) and use Mellin inversion to estimate the product of each factor with the corresponding Archimedean term in $\left|a_{f}(1)\right|^{2}$. For $i \leq r_{1}$ we need to estimate

$$
\frac{(4 \pi)^{k_{i}}}{\Gamma\left(k_{i}\right)} \int_{\mathbb{R}^{+}} h\left(T\left|\eta_{i} \kappa_{i}\right|^{-1} y\right) y^{k_{i}-1} \exp (-4 \pi y) d y^{\times},
$$

which by Mellin inversion is equal to

$$
\frac{4 \pi}{k_{i}-1} \int_{(\sigma)} H(-s)\left(\frac{T}{4 \pi\left|\eta_{i} \kappa_{i}\right|}\right)^{s} \frac{\Gamma\left(s+k_{i}-1\right)}{\Gamma\left(k_{i}-1\right)} d s .
$$

Applying the Luo-Sarnak lemma to this, we have

$$
\begin{aligned}
(59) & =\frac{4 \pi}{k_{i}-1} \int_{(\sigma)} H(-s)\left(\frac{T}{4 \pi\left|\eta_{i} \kappa_{i}\right|}\right)^{s}\left(k_{i}-1\right)^{s}\left(1+O\left((|s|+1)^{2} k_{i}^{-1}\right)\right) d s \\
& \ll \frac{1}{k_{i}}\left(h\left(\frac{T\left(k_{i}-1\right)}{4 \pi\left|\eta_{i} \kappa_{i}\right|}\right)+\left|\int_{(\sigma)} H(-s)\left(\frac{T}{4 \pi\left|\eta_{i} \kappa_{i}\right|}\right)^{s}\left(k_{i}-1\right)^{s-1}(|s|+1)^{2} d s\right|\right) .
\end{aligned}
$$

Choosing $\sigma=1+\epsilon$ gives the required bound:

$$
\text { (59) } \ll \frac{1}{k_{i}}\left(h\left(\frac{T\left(k_{i}-1\right)}{4 \pi\left|\eta_{i} \kappa_{i}\right|}\right)+O\left(k_{i}^{\epsilon}\left(T /\left|\eta_{i}\right|\right)^{1+\epsilon}\right)\right) .
$$

For $i>r_{1}$, we must estimate

$$
\frac{(2 \pi)^{k_{i}}}{\Gamma\left(k_{i} / 2+1\right)^{2}}\left(\begin{array}{c}
k_{i} \\
j_{i}
\end{array}\right) \int_{\mathbb{R}^{+}} h\left(T\left|\eta_{i} \kappa_{i}\right|^{-1} y\right) y^{k_{i}}\left|K_{k_{i} / 2-j_{i}}(4 \pi y)\right|^{2} d y^{\times} .
$$

Applying Mellin inversion using (56), this becomes

$$
\begin{aligned}
\ll \frac{1}{\Gamma\left(k_{i} / 2+1\right)^{2}}\left(\begin{array}{c}
k_{i} \\
j_{i}
\end{array}\right) \int_{\sigma} H(-s)\left(\frac{T}{2 \pi\left|\eta_{i} \kappa_{i}\right|}\right)^{s} & \frac{\Gamma\left(\left(s+k_{i}\right) / 2\right)^{2}}{\Gamma\left(s+k_{i}\right)} \\
& \times \Gamma\left(s / 2+j_{i}\right) \Gamma\left(s / 2+k_{i}-j_{i}\right) d s .
\end{aligned}
$$


Because $j \in J_{0}$, we have that $j_{i}$ and $k_{i}-j_{i}$ are $\geq k_{i}^{1 / 2}$, so we may apply (34) and choose $\sigma=2+\epsilon$ to obtain

$$
\left(\underline{62)} \ll \frac{1}{k_{i}\left(k_{i}-j_{i}\right) j_{i}} h\left(\frac{T \sqrt{j_{i}\left(k_{i}-j_{i}\right)}}{2 \pi\left|\eta_{i} \kappa_{i}\right|}\right)+O\left(k_{i}^{-3 / 2+\epsilon}\left(T /\left|\eta_{i}\right|\right)^{2+\epsilon}\right) .\right.
$$

After substituting the bounds (61) and (63), equation (51) becomes

$$
\begin{aligned}
S_{\xi, j}(T) \ll & \frac{\left|a_{\xi}\left(T^{-1}\right)\right|}{L\left(1, \operatorname{sym}^{2} \pi\right)} \sum_{\eta>0}\left|\lambda_{\pi}(\eta) \lambda_{\pi}(\eta+\xi)\right| \\
& \times \prod_{i \leq r_{1}} \frac{1}{k_{i}}\left(h\left(\frac{T\left(k_{i}-1\right)}{4 \pi\left|\eta_{i} \kappa_{i}\right|}\right)+O\left(k_{i}^{\epsilon}\left(T /\left|\eta_{i}\right|\right)^{1+\epsilon}\right)\right) \\
& \times \prod_{i>r_{1}}\left(\frac{1}{k_{i}\left(k_{i}-j_{i}\right) j_{i}} h\left(\frac{T \sqrt{j_{i}\left(k_{i}-j_{i}\right)}}{2 \pi\left|\eta_{i} \kappa_{i}\right|}\right)+O\left(k_{i}^{-3 / 2+\epsilon}\left(T /\left|\eta_{i}\right|\right)^{2+\epsilon}\right)\right) .
\end{aligned}
$$

As in the totally real case we may use the bound $\left|\lambda_{\pi}(\eta)\right| \ll N \eta^{\epsilon}$ to show that the contribution to the sum from all error terms is $O\left(|J|^{-1}\|k\|^{-\nu / 2+\epsilon} T^{n+\epsilon}\right)$, so our upper bound may be rewritten as

$$
\begin{aligned}
S_{\xi, j}(T) \ll & \frac{\left|a_{\xi}\left(T^{-1}\right)\right|}{L\left(1, \operatorname{sym}^{2} \pi\right)}\left(\sum_{\eta>0}\left|\lambda_{\pi}(\eta) \lambda_{\pi}(\eta+\xi)\right| \prod_{i \leq r_{1}} \frac{1}{k_{i}} h\left(\frac{T\left(k_{i}-1\right)}{4 \pi\left|\eta_{i} \kappa_{i}\right|}\right)\right. \\
& \left.\times \prod_{i>r_{1}} \frac{1}{k_{i}\left(k_{i}-j_{i}\right) j_{i}} h\left(\frac{T \sqrt{j_{i}\left(k_{i}-j_{i}\right)}}{2 \pi\left|\eta_{i} \kappa_{i}\right|}\right)+O\left(|J|^{-1}\|k\|^{-\nu / 2+\epsilon} T^{n+\epsilon}\right)\right) .
\end{aligned}
$$

On summing over $j$ it can be seen that we have proven the inequalities (43) and (44), where the terms for $j \in J_{1}$ are absorbed into the error term in (43).

6.3. Extracting shifted convolution sums: The zero shift. Having dealt with the 'error' terms with $\xi \neq 0$, it remains to prove the estimates (41) and (42) for the 'main' term $S_{0}(T)$, which by (45) reduces to studying the integral $I_{1}(T)$ as in the totally real case.

Squaring out $\left|F_{k}(z)\right|^{2}$ and integrating in $x$, we obtain

$$
I_{1}(T)=2^{-r_{2}} \sqrt{|D|} \sum_{\eta>0}\left|a_{f}(\eta)\right|^{2} \int_{\mathbb{R}_{+}^{r}} g(T y)\left|\mathbf{K}_{k}(\eta \kappa y)\right|^{2} N y^{-1} d y^{\times} .
$$

Applying the normalisation of $a_{f}(\eta)$, we have

$$
I_{1}(T)=2^{-r_{2}} \sqrt{|D|}\left|a_{f}(1)\right|^{2} N \kappa \sum_{\eta>0}\left|\lambda_{\pi}(\eta)\right|^{2} \int_{\mathbb{R}_{+}^{r}} g(T y) N(\eta \kappa y)^{-1}\left|\mathbf{K}_{k}(\eta \kappa y)\right|^{2} d y^{\times} .
$$

As with the nonzero shifts, we may expand this into a sum over the multi-indices $j \in J$, and denote the $j$ th term by $I_{1, j}(T)$. If we define the symmetrised functions $\widetilde{g}$ and $\widetilde{\psi}_{j}$ by

$$
\widetilde{g}(y)=\sum_{u \in \mathcal{O}_{+}^{\times}} g(u y), \quad \widetilde{\psi}_{j}(y)=N y^{-1} \sum_{u \in \mathcal{O}_{+}^{\times}}\left|K_{k, j}(u y)\right|^{2},
$$


then $I_{1, j}(T)$ may be expressed as

$$
\begin{aligned}
I_{1, j}(T) & =2^{-r_{2}}\left|\mu_{+}\right| \sqrt{|D|}\left|a_{f}(1)\right|^{2} N \kappa \sum_{(\eta)>0}\left|\lambda_{\pi}(\eta)\right|^{2} \int_{\mathbb{R}_{+}^{r}} g(T y) \widetilde{\psi}_{j}(\eta \kappa y) d y^{\times} \\
& =2^{-r_{2}}\left|\mu_{+}\right| \sqrt{|D|}\left|a_{f}(1)\right|^{2} N \kappa \sum_{(\eta)>0}\left|\lambda_{\pi}(\eta)\right|^{2} \int_{\mathbb{R}_{+}^{r} / \mathcal{O}_{+}^{\times}} \widetilde{g}(T y) \widetilde{\psi}_{j}(\eta \kappa y) d y^{\times} .
\end{aligned}
$$

Note that the factor of $\left|\mu_{+}\right|$arises because the quotient of $\mathcal{O}^{+}$by $\mathcal{O}_{+}^{\times}$contains each ideal with this multiplicity. If we let $G(s, m)$ be the Mellin transform of $\widetilde{g}$ as in (23), then Mellin inversion gives

$$
\begin{aligned}
& \int_{\mathbb{R}_{+}^{r} / \mathcal{O}_{+}^{\times}} \tilde{g}(T y) \widetilde{\psi}_{j}(\eta \kappa y) d y^{\times} \\
& \quad=\frac{1}{2 \pi i V_{c}} \sum_{m} \int_{(\sigma)} G(-s,-m)\left(\frac{T}{4 \pi}\right)^{n s} N(\eta \kappa)^{-s} \lambda_{-m}(\eta \kappa) \Gamma(k, j, s, m) d s,
\end{aligned}
$$

where $\Gamma(k, j, s, m)$ is the Mellin transform of $\widetilde{\psi}_{j}$, which may be calculated using (56) to be

$$
\begin{aligned}
& \Gamma(k, j, s, m)= \prod_{i \leq r_{1}}(4 \pi)^{-k_{i}+1} \Gamma\left(s+\beta(m, i)+k_{i}-1\right) \\
& \times \prod_{i>r_{1}}(2 \pi)^{-k_{i}}\left(\begin{array}{c}
k_{i} \\
j_{i}
\end{array}\right) 2^{2 s+\beta(m, i)} \frac{\Gamma\left(s+\left(\beta(m, i)+k_{i}\right) / 2\right)^{2}}{8 \Gamma\left(2 s+\beta(m, i)+k_{i}\right)} \\
& \quad \times \Gamma\left(s+\beta(m, i) / 2+j_{i}\right) \Gamma\left(s+\beta(m, i) / 2+k_{i}-j_{i}\right) .
\end{aligned}
$$

Substituting (64) into $I_{1, j}(T)$ and forming the $L$-function from the sum over $\eta$, we have

$$
\begin{aligned}
I_{1, j}(T)=\frac{\left|\mu_{+}\right| \sqrt{|D|}}{2^{r_{2}} V_{c}}\left|a_{f}(1)\right|^{2} & \frac{1}{2 \pi i} \sum_{m} \int_{(\sigma)} G(-s,-m)\left(\frac{T}{4 \pi}\right)^{n s} N \kappa^{1-s} \lambda_{-m}(\kappa) \\
& \times L\left(s, \operatorname{sym}^{2} \pi \otimes \lambda_{-m}\right) \frac{L\left(s, \lambda_{-m}\right)}{L\left(2 s, \lambda_{-2 m}\right)} \Gamma(k, j, s, m) d s .
\end{aligned}
$$

We now substitute the value of $\left|a_{f}(1)\right|^{2}$ and shift the line of integration to $\sigma=$ $1 / 2$, giving

$$
I_{1, j}(T)=\frac{C_{g} T^{n}}{|J|}+E_{1 / 2, j}(T)
$$

with

$$
\begin{aligned}
& E_{1 / 2, j}(T) \ll \frac{1}{L\left(1, \mathrm{sym}^{2} \pi\right)} \prod_{i \leq r_{1}} \frac{(4 \pi)^{k_{i}}}{\Gamma\left(k_{i}\right)} \prod_{i>r_{1}} \frac{(2 \pi)^{k_{i}}}{\Gamma\left(k_{i} / 2+1\right)^{2}}\left(\begin{array}{c}
k_{i} \\
j_{i}
\end{array}\right) \\
& \times \sum_{m} \int_{(1 / 2)} G(-s,-m)\left(\frac{T}{4 \pi}\right)^{n s} L\left(s, \operatorname{sym}^{2} \pi \otimes \lambda_{-m}\right) \frac{L\left(s, \lambda_{-m}\right)}{L\left(2 s, \lambda_{-2 m}\right)} \Gamma(k, j, s, m) d s .
\end{aligned}
$$


By Stirling's formula and the rapid decay of $G(s, m)$ this error term may be bounded above by

$$
\begin{aligned}
E_{1 / 2, j}(T) \ll\left(\frac{T^{n}}{N k}\right)^{1 / 2} \frac{1}{L\left(1, \mathrm{sym}^{2} \pi\right)} \prod_{i>r_{1}} \frac{1}{\sqrt{j_{i}\left(k_{i}-j_{i}\right)}} \\
\quad \times \sum_{m} \int_{-\infty}^{+\infty} \frac{\left|L\left(1 / 2+i t, \mathrm{sym}^{2} \pi \otimes \lambda_{-m}\right)\right|}{(|t|+\|m\|+1)^{A}} d t
\end{aligned}
$$

for any $A>0$. Because $x^{-1 / 2}$ is integrable at 0 ,

$$
\sum_{j \in J} \prod_{i>r_{1}} \frac{1}{\sqrt{j_{i}\left(k_{i}-j_{i}\right)}}
$$

is bounded independently of $k$ so that when we sum (65) and (66) over $j$ we obtain

$$
I_{1}(T)=C_{g} T^{n}+O\left(T^{n / 2} R_{k}(\pi)\right),
$$

with

$$
R_{k}(\pi)=\frac{1}{\sqrt{N k} L\left(1, \operatorname{sym}^{2} \pi\right)} \sum_{m} \int_{-\infty}^{+\infty} \frac{\left|L\left(1 / 2+i t, \operatorname{sym}^{2} \pi \otimes \lambda_{-m}\right)\right|}{(|t|+\|m\|+1)^{A}} d t .
$$

This completes the proof of Proposition 13 .

\section{Application of the large sieve}

In this section we complete the proof of Theorem 4 by establishing the bounds of Proposition 10 for the shifted sums

$$
C_{\xi}(x)=\sum_{\eta \leq x}\left|\lambda_{1}(\eta) \lambda_{2}(\eta+\xi)\right|,
$$

where the $\lambda_{i}$ are multiplicative functions on $\mathcal{O}^{+}$satisfying $\left|\lambda_{i}(\eta)\right| \leq \tau_{m}(\eta)$ for some $m$ and $x=\left(x_{i}\right)$ satisfies $x_{i} \geq\|x\|^{\nu}$ for some $\nu>0$. We first rearrange and partition the sums into pieces which may be treated either by elementary methods or by a large sieve. We assume that $0<\|\xi\| \leq\|x\|^{\nu}$, and given $\epsilon>0$ we will be working throughout with a choice of variables satisfying

$$
\begin{aligned}
& z=\|x\|^{1 / s} \quad \text { with } s=\epsilon \log \log \|x\|, \\
& y=\|x\|^{\epsilon} .
\end{aligned}
$$

We factorise the ideals $(\eta)$ and $(\eta+\xi)$ as

$$
(\eta)=\mathfrak{a} \mathfrak{b} \quad \text { and } \quad(\eta+\xi)=\mathfrak{a}_{\xi} \mathfrak{b}_{\xi}
$$

in such a way that for every prime ideal $\mathfrak{p}$ dividing $\eta(\eta+\xi)$,

$$
\mathfrak{p} \mid \mathfrak{a} \mathfrak{a}_{\xi} \Rightarrow N \mathfrak{p} \leq z \quad \text { and } \quad \mathfrak{p} \mid \mathfrak{b} \mathfrak{b}_{\xi} \Rightarrow N \mathfrak{p}>z
$$

and partition the sum $C_{\xi}(x)$ into parts depending on the norm of $\mathfrak{a}$ and $\mathfrak{a}_{\xi}$. We denote by $C^{y}(x)$ the part of $C_{\xi}(x)$ in which either $N \mathfrak{a}$ or $N \mathfrak{a}_{\xi}$ is greater than $y$,

$$
C^{y}(x)=\sum_{\substack{\eta \leq x \\ \max \left\{N \mathfrak{a}, N \mathfrak{a}_{\xi}\right\}>y}}\left|\lambda_{1}(\eta) \lambda_{2}(\eta+\xi)\right|,
$$


and the part where both $N \mathfrak{a}$ and $N \mathfrak{a}_{\xi}$ are less than or equal to $y$ we denote by $C_{y}(x)$,

$$
C_{y}(x)=\sum_{\substack{\eta \leq x \\ N \mathfrak{a}, N \mathfrak{a}_{\xi} \leq y}}\left|\lambda_{1}(\eta) \lambda_{2}(\eta+\xi)\right|
$$

so that $C_{\xi}(x)=C^{y}(x)+C_{y}(x)$.

7.1. Treating $C^{y}(x)$ by elementary methods. We first handle the terms with $N \mathfrak{a}$ or $N \mathfrak{a}_{\xi}$ large. We may assume without loss of generality that $N \mathfrak{a}>y$, and begin by applying Hölder's inequality and $\left|\lambda_{i}(\eta)\right| \leq \tau_{m}(\eta)$ to obtain

$$
C^{y}(x) \ll\left(\sum_{\substack{\eta \leq x \\ N \mathfrak{a}>y}} 1\right)^{1 / 2}\left(\sum_{\eta \leq x+\|\xi\|} \tau_{m}^{4}(\eta)\right)^{1 / 2} .
$$

We know that $x+\|\xi\| \leq 2 x$ by our assumption on $\xi$, and we have the bound

$$
\sum_{\eta \leq 2 x} \tau_{m}^{4}(\eta) \ll N x(\log \|x\|)^{A}
$$

for some $A$. By a classical Rankin's method argument ([26, Thm. 7.6), the number of ideals with norm at most $N x$, containing only small prime factors, can be bounded by $\ll N x(\log \|x\|)^{-A}$ for any $A>0$. Partial summation and our choice of $x, y$ and $z$ then gives the bound

$$
\sum_{\substack{(\eta): N \eta \leq N x \\ N \mathfrak{a}>y}} 1 \ll \frac{N x}{(\log \|x\|)^{A}}
$$

for any $A$, and the upper bound of $(\log \|x\|)^{n-1}$ for the number of $\eta \leq x$ generating a given $(\eta)$ lets us conclude

$$
C^{y}(x) \leq \frac{N x}{(\log \|x\|)^{2}} .
$$

7.2. Treating $C_{y}(x)$ by the large sieve. By our definition of $C_{y}(x)$, we are left with evaluating

$$
C_{y}(x)=\sum_{\substack{N \mathfrak{a}, N \mathfrak{a} \mathfrak{q}_{\xi} \leq y \\ \mathfrak{p} \mid \mathfrak{a} \mathfrak{a}_{\xi} \Rightarrow N \mathfrak{p} \leq z}}\left|\lambda_{1}(\mathfrak{a}) \lambda_{2}\left(\mathfrak{a}_{\xi}\right)\right| \sum_{\substack{\eta \leq x \\ \eta \equiv 0(\mathfrak{a}) \\ \eta \equiv-\xi\left(\mathfrak{a}_{\xi}\right) \\ \mathfrak{p} \mid \mathfrak{b} \mathfrak{b}_{\xi} \Rightarrow N \mathfrak{p}>z}}\left|\lambda_{1}(\mathfrak{b}) \lambda_{2}\left(\mathfrak{b}_{\xi}\right)\right| .
$$

To help deal with certain co-primality conditions which come up during our analysis, we pull out the greatest common divisor $\mathfrak{v}$ of $\mathfrak{a}$ and $\mathfrak{a}_{\xi}$, which we choose to have a normalised positive generator $v$. Writing $\eta_{v}=\eta / v$ and $\eta_{v}+w=(\eta+\xi) / v$, we again factorise $\left(\eta_{v}\right)$ and $\left(\eta_{v}+w\right)$ as

$$
\left(\eta_{v}\right)=\mathfrak{a} \mathfrak{b} \quad \text { and } \quad\left(\eta_{v}+w\right)=\mathfrak{a}_{\xi} \mathfrak{b}_{\xi}
$$

with $\left(\mathfrak{a}, \mathfrak{a}_{\xi}\right)=\left(\mathfrak{a} \mathfrak{a}_{\xi}, w\right)=\mathcal{O}$, so that

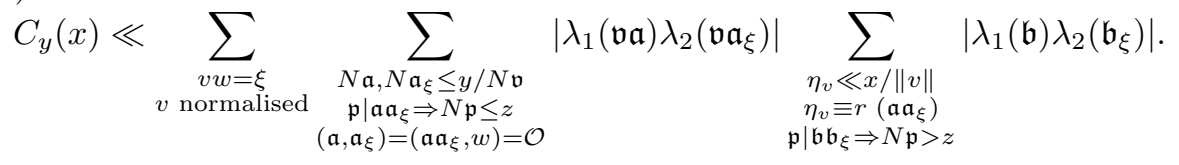


Here we applied the Chinese Remainder Theorem so that the residue class $r$ in the innermost sum satisfies $r \equiv 0(\bmod \mathfrak{a})$ and $r \equiv-w\left(\bmod \mathfrak{a}_{\xi}\right)$. The Ramanujan bound and our choice of $s$ in (67) imply that $\left|\lambda_{1}(\mathfrak{b}) \lambda_{2}\left(\mathfrak{b}_{\xi}\right)\right| \ll(\log \|x\|)^{2 m \epsilon}$, as we have $\left|\lambda_{1}\left(\mathfrak{p}^{\alpha}\right)\right| \leq \tau_{m}\left(\mathfrak{p}^{\alpha}\right) \leq 2^{\alpha+m-1}$ and $\mathfrak{b}=\mathfrak{p}_{1}^{\alpha_{1}} \ldots \mathfrak{p}_{t}^{\alpha_{t}}$ with $\alpha_{1}+\cdots+\alpha_{t} \leq s$. We may therefore substitute this in (70), and proceed to bound the count

$$
\sum_{\substack{\eta_{v} \ll x /\|v\| \\ \eta_{v} \equiv r\left(\mathfrak{a} \mathfrak{a}_{\xi}\right) \\ \mathfrak{p} \mid \mathfrak{b} \mathfrak{b}_{\xi} \Rightarrow N \mathfrak{p}>z}} 1 .
$$

Choose normalised generators $a$ and $a_{\xi}$ for $\mathfrak{a}$ and $\mathfrak{a}_{\xi}$, which will satisfy $\|a\|,\left\|a_{\xi}\right\|$ $\ll y^{1 / n} /\|v\|$. Writing $\eta_{v}=a a_{\xi} m+r$ with $r$ chosen in a negative fundamental domain for $\mathbb{F} / \mathfrak{a} \mathfrak{a}_{\xi}$ (so that $a a_{\xi} m \geq 0$ ), we note the following equivalences between divisibility conditions for primes $\mathfrak{p}$ with $N \mathfrak{p} \leq z$ :

$$
\begin{aligned}
\mathfrak{p} \nmid \mathfrak{b} & \Longleftrightarrow \mathfrak{p} \nmid\left(a_{\xi} m+r / a\right), \\
\mathfrak{p} \nmid \mathfrak{b}_{\xi} & \Longleftrightarrow \mathfrak{p} \nmid\left(a m+(r+w) / a_{\xi}\right) .
\end{aligned}
$$

For fixed normalised $a$ and $a_{\xi}$ satisfying $\left(a, a_{\xi}\right)=\left(a a_{\xi}, w\right)=\mathcal{O}$ and $\|a\|,\left\|a_{\xi}\right\| \ll$ $y^{1 / n} /\|v\|$, we see that the count in (71) is bounded by $S=|\mathcal{S}(\mathcal{M}, \mathcal{P}, \Omega)|$, where we define $\mathcal{S}(\mathcal{M}, \mathcal{P}, \Omega)$ to be the 'sifted set',

$$
\mathcal{S}(\mathcal{M}, \mathcal{P}, \Omega)=\left\{m \in \mathcal{M}: m(\bmod \mathfrak{p}) \notin \Omega_{\mathfrak{p}} \text { for all } \mathfrak{p} \in \mathcal{P}\right\} .
$$

Here,

$$
\begin{aligned}
\mathcal{M} & =\left\{m \in \mathcal{O}: 0<\text { mvaa }_{\xi} \ll x\right\} \\
\mathcal{P} & =\{\mathfrak{p}: 2<N \mathfrak{p} \leq z\},
\end{aligned}
$$

and the set $\Omega=\bigcup_{\mathfrak{p} \in \mathcal{P}} \Omega_{\mathfrak{p}}$ of residue classes to be 'sieved out' is given by

$$
\Omega_{\mathfrak{p}}= \begin{cases}\left\{r_{1}(\bmod \mathfrak{p})\right\} & \text { for } \mathfrak{p} \mid a, \\ \left\{r_{2}(\bmod \mathfrak{p})\right\} & \text { for } \mathfrak{p} \mid a_{\xi}, \\ \left\{r_{1}, r_{2}(\bmod \mathfrak{p})\right\} & \text { for } \mathfrak{p} \nmid a a_{\xi},\end{cases}
$$

where $r_{1} \equiv-\overline{a_{\xi}} r / a(\bmod \mathfrak{p})$ and $r_{2} \equiv-\bar{a}(r+w) / a_{\xi}(\bmod \mathfrak{p})$. Here the overline means multiplicative inverse $\bmod \mathfrak{p}$.

We now apply a variant of the standard large sieve for the lattice $\mathbb{Z}^{n}$. Let $d=\left(d_{i}\right)$ with $d_{i}>\|d\|^{\nu}$ for some $\nu>0$, let $B(d)$ be the box with dimensions $d$ centred at the origin in $\mathbb{R}^{n}$, and let $D(d)$ be the image of $B(d)$ under any rotation. If $\mathcal{P}$ is a set of rational primes, let $\Omega_{p}$ be a subset of $L / p L$ of cardinality $\omega(p)$ for each $p \in \mathcal{P}$, and define a sifted set $\mathcal{S}(\mathcal{L}, \mathcal{P}, \Omega)$ by

$$
\begin{aligned}
\mathcal{S}(\mathcal{L}, \mathcal{P}, \Omega) & =\left\{m \in \mathcal{L}: m(\bmod p) \notin \Omega_{p} \text { for all } p \in \mathcal{P}\right\}, \\
\text { with } \mathcal{L} & =\mathbb{Z}^{n} \cap D(d) .
\end{aligned}
$$

We then have

$$
|\mathcal{S}(\mathcal{L}, \mathcal{P}, \Omega)| \ll_{\nu} \frac{N d+Q^{2 n}}{H}
$$

for any $\|d\|^{\nu / 2} \geq Q \geq 1$, where

$$
H=\sum_{q \leq Q} h(q)
$$


and $h(q)$ is the multiplicative function supported on squarefree integers with prime divisors in $\mathcal{P}$ such that

$$
h(p)=\frac{\omega(p)}{p^{n}-\omega(p)} .
$$

This form of the large sieve may be proven using soft techniques of Poisson summation, described in chapter 7 of [18].

To apply this in the number field, identify $\mathcal{O}$ with $\mathbb{Z}^{n}$ and for each $p$, construct a set $\Omega_{p}$ from the $\Omega_{\mathfrak{p}}$ with $\mathfrak{p} \mid p$ using the Chinese Remainder Theorem. We then have

$$
\omega(p) \geq\left(\alpha_{p}+\beta_{p}\right) p^{n-1}+O\left(p^{n-2}\right),
$$

where $\alpha_{p}$ is the number of degree 1 primes above $p$ and $\beta_{p}$ is the number which do not divide $a a_{\xi}$. Choosing $Q=z$ gives

$$
\begin{gathered}
H \gg_{\nu} \sum_{n \leq z} \frac{a_{n}}{n}, \\
\text { where } \sum \frac{a_{n}}{n}=\prod_{N \mathfrak{p} \leq z}\left(1+\frac{2}{N \mathfrak{p}}\right) \prod_{\mathfrak{p} \mid a a_{\xi}}\left(1-\frac{1}{N \mathfrak{p}}\right) .
\end{gathered}
$$

This implies that

$$
\begin{aligned}
H & \gg_{\nu} \prod_{\mathfrak{p} \mid a a_{\xi}}\left(1-\frac{1}{N \mathfrak{p}}\right) \sum_{n \leq z} \frac{b_{n}}{n}, \\
\text { where } \sum \frac{b_{n}}{n} & =\prod_{N \mathfrak{p} \leq z}\left(1+\frac{2}{N \mathfrak{p}}\right) .
\end{aligned}
$$

Applying standard Tauberian estimates allows us to conclude that

$$
H \gg_{\nu}(\log z)^{2} \prod_{\mathfrak{p} \mid a a_{\xi}}\left(1-\frac{1}{N \mathfrak{p}}\right),
$$

so that the count (71) is bounded by

$$
\ll_{\nu} \frac{N x}{(\log z)^{2} N\left(v a a_{\xi}\right)} \prod_{\mathfrak{p} \mid a a_{\xi}}\left(1-\frac{1}{N \mathfrak{p}}\right)^{-1} .
$$

Plugging this back into (70), we obtain

$$
\begin{aligned}
C^{y}(x) \ll_{\nu} \frac{(\log \|x\|)^{2 m \epsilon} N x}{(\log z)^{2}} \sum_{\begin{array}{c}
v w=\xi \\
v \text { normalised }
\end{array}} \sum_{\begin{array}{c}
N \mathfrak{a}, N \mathfrak{a}_{\xi} \leq y / N \mathfrak{v} \\
\mathfrak{p} \mid \mathfrak{a} \mathfrak{a}_{\xi} \Rightarrow N \mathfrak{p} \leq z \\
\left(\mathfrak{a}, \mathfrak{a}_{\xi}\right)=\left(\mathfrak{a} \mathfrak{a}_{\xi}, w\right)=\mathcal{O}
\end{array}} \frac{\left|\lambda_{1}(\mathfrak{v a}) \lambda_{2}\left(\mathfrak{v a} \mathfrak{a}_{\xi}\right)\right|}{N\left(v a a_{\xi}\right)} \\
\times \prod_{\mathfrak{p} \mid a a_{\xi}}\left(1-\frac{1}{N \mathfrak{p}}\right)^{-1} .
\end{aligned}
$$

For each $v$, we may bound the inner sum from above by an Euler product. If $\mathfrak{p} \backslash v$, the corresponding term is

$$
1+\frac{\left|\lambda_{1}(\mathfrak{p})\right|+\left|\lambda_{2}(\mathfrak{p})\right|}{N \mathfrak{p}}+O\left(N \mathfrak{p}^{-2+\epsilon}\right)
$$


by our bounds on $\left|\lambda_{i}(\mathfrak{p})\right|$, and if $\mathfrak{p} \mid v$ it is

$$
\frac{\left|\lambda_{1}(\mathfrak{p}) \lambda_{2}(\mathfrak{p})\right|}{N \mathfrak{p}}+O\left(N \mathfrak{p}^{-2+\epsilon}\right) .
$$

(73) is at most 1 for almost all $\mathfrak{p}$, and so for any $v$ we may bound the inner sum by

$$
\ll_{\nu} \prod_{N \mathfrak{p} \leq z}\left(1+\frac{\left|\lambda_{1}(\mathfrak{p})\right|+\left|\lambda_{2}(\mathfrak{p})\right|}{N \mathfrak{p}}\right) .
$$

This gives the bound

$$
C^{y}(x) \ll_{\nu} \frac{\tau(\xi) N x}{(\log \|x\|)^{2-\epsilon}} \prod_{N \mathfrak{p} \leq z}\left(1+\frac{\left|\lambda_{1}(\mathfrak{p})\right|+\left|\lambda_{2}(\mathfrak{p})\right|}{N \mathfrak{p}}\right)
$$

for $C^{y}(x)$, and when combined with our partition of $C_{\xi}(x)$ and the bound (69) this concludes the proof of Proposition 10.

\section{Proof of Theorem 5}

In this section we shall prove Theorem 5 by extending Soundararajan's approach of weak subconvexity to a number field. We prove the necessary triple product identities in section 8.1. before showing that the triple product $L$-functions which appear there satisfy the hypotheses of Soundararajan's theorem in section 8.2 .

8.1. Triple products. Throughout this section, $C$ will denote a constant depending only on $F$ which may vary from equation to equation. We shall also let $\sigma$ denote the conjugate linear automorphism of $\pi$ corresponding to complex conjugation on $X$, which has the property that $\langle\sigma(u), \sigma(v)\rangle=\overline{\langle u, v\rangle}$. The first triple product identity we shall present deals with the case of $\phi$ being a Hecke-Maass cusp form.

Proposition 14. Let $\phi$ be a Hecke-Maass cusp form with associated automorphic representation $\pi^{\prime}$. Then

$$
\begin{aligned}
\left|\left\langle\phi F_{k}, F_{k}\right\rangle\right|^{2} & =C \frac{\Lambda\left(\frac{1}{2}, \pi \otimes \pi \otimes \pi^{\prime}\right)}{\Lambda\left(1, \operatorname{sym}^{2} \pi\right)^{2} \Lambda\left(1, \mathrm{sym}^{2} \pi^{\prime}\right)} \\
& \sim_{\phi} \quad N k^{-1} \frac{L\left(\frac{1}{2}, \mathrm{sym}^{2} \pi \otimes \pi^{\prime}\right)}{L\left(1, \mathrm{sym}^{2} \pi\right)^{2}}
\end{aligned}
$$

where $\sim_{\phi}$ means that the ratio of the two quantities is bounded between two positive constants depending only on $\phi$.

Proof. Because $\left|F_{k}\right|^{2} d v$ is the pushforward of $\left|R_{\pi}\left(v_{k}\right)\right|^{2} d x$, the inner product $\left\langle\phi F_{k}\right.$, $\left.F_{k}\right\rangle$ is equal to

$$
\int_{X}\left|R_{\pi}\left(v_{k}\right)\right|^{2} \phi d x=\int_{X} R_{\pi}\left(v_{k}\right) R_{\pi}\left(\sigma\left(v_{k}\right)\right) \phi d x,
$$

and we may evaluate the RHS of this expression using Ichino's formula. Let $I=$ $\otimes I_{i}$ and $I^{\prime}=\otimes I_{i}^{\prime}$ be the products of the Archimedean local factors of $\pi$ and $\pi^{\prime}$, let $k_{i}$ and $r_{i}^{\prime}$ be the relevant parameters of these local factors, and let $u \in I^{\prime}$ be a 
unit spherical vector. As all our vectors are unramified and our division algebra is split, the statement of Ichino's formula in this case is

$$
\begin{aligned}
& \left|\int_{X} R_{\pi}\left(v_{k}\right) R_{\pi}\left(\sigma\left(v_{k}\right)\right) \phi d x\right|^{2} \\
& =C \prod_{j=1}^{r} \int_{\overline{G_{j}}}\left\langle I_{j}(g) v_{k_{j}}, v_{k_{j}}\right\rangle\left\langle I_{j}(g) v_{-k_{j}}, v_{-k_{j}}\right\rangle\left\langle I_{j}^{\prime}(g) u_{j}, u_{j}\right\rangle d \overline{g_{j}} \\
& \quad \times \frac{L\left(\frac{1}{2}, \pi \otimes \pi \otimes \pi^{\prime}\right)}{L\left(1, \mathrm{sym}^{2} \pi\right)^{2} L\left(1, \mathrm{sym}^{2} \pi^{\prime}\right)},
\end{aligned}
$$

where $v_{k}=\bigotimes v_{k_{j}}$ and $u=\bigotimes u_{j}$. If $\nu_{j}$ is a complex place the $j$ th local integral appearing in the product was computed in [24] to be

$$
C \frac{\left|\Gamma\left(\left(1+k_{j}+i r_{j}^{\prime}\right) / 2\right) \Gamma\left(\left(1+i r_{j}^{\prime}\right) / 2\right)\right|^{4}}{\Gamma\left(1+k_{j} / 2\right)^{4}\left|\Gamma\left(1+i r_{j}^{\prime}\right)\right|^{2}},
$$

and up to an absolute constant this is equal to the ratio of the Archimedean factors at the place $\nu_{j}$ of the $L$-functions appearing in (76). In the real case the local integral may be determined by comparison with Watson's formula, and is

$$
C \frac{\left|\Gamma\left(k_{j}-1 / 2+i r_{j}^{\prime}\right) \Gamma\left(1 / 2+i r_{j}^{\prime}\right)\right|^{2}}{\Gamma\left(k_{j}-1 / 2\right)^{2}\left|\Gamma\left(1 / 4+i r_{j}^{\prime}\right)\right|^{2}} .
$$

This is again proportional to the relevant Archimedean factors of the $L$-functions appearing in (76), which gives (74). Finally, the Archimedean factors (78) and (77) have the asymptotic behaviours $k_{j}^{-1}$ and $k_{j}^{-2}$ as $k_{j} \rightarrow \infty$, which gives (75).

We now treat the inner products $\left\langle E(s, m, \cdot) F_{k}, F_{k}\right\rangle$ against spherical Eisenstein series by unfolding.

\section{Proposition 15.}

$$
\left|\left\langle E(1 / 2+i t, m, \cdot) F_{k}, F_{k}\right\rangle\right|=C\left|\frac{\Lambda\left(1 / 2+i t, \pi \otimes \pi \otimes \lambda_{-m}\right)}{\Lambda\left(1, \operatorname{sym}^{2} \pi\right) \Lambda\left(1+2 i t, \lambda_{-2 m}\right)}\right|,
$$

$$
\left|\left\langle E(1 / 2+i t, m, \cdot) F_{k}, F_{k}\right\rangle\right| \ll \frac{(1+|t|+\|m\|)^{n / 4+\epsilon}}{N k}\left|\frac{L\left(1 / 2+i t, \mathrm{sym}^{2} \pi \otimes \lambda_{-m}\right)}{L\left(1, \mathrm{sym}^{2} \pi\right)}\right| .
$$

Proof. For $\operatorname{Re}(s)>1$, unfolding and substituting the Fourier expansion of $F_{k}$ gives

$$
\begin{aligned}
\left\langle E(s, m, \cdot) F_{k}, F_{k}\right\rangle & =\int_{\Gamma_{\infty} \backslash \mathbb{H}_{F}} N y^{s} \lambda_{m}(y)\left|F_{k}(z)\right|^{2} d v \\
& =\left|a_{f}(1)\right|^{2} \int_{\Gamma_{\infty} \backslash \mathbb{H}_{F}} N y^{s} \lambda_{m}(y) \sum_{\xi \in \mathcal{O}}\left|\lambda_{\pi}(\xi)\right|^{2} N \xi^{-1}\left|\mathbf{K}_{k}(\xi \kappa y)\right|^{2} d v .
\end{aligned}
$$

We know that $\Gamma_{\infty} \backslash \mathbb{H}_{F}$ is isomorphic to $\mathbb{R}_{+}^{r} / \mathcal{O}_{+}^{\times} \times \mathbb{F} / \mathcal{O} \mu_{+}$, where $\mathbb{F} / \mathcal{O} \mu_{+}$denotes the quotient of $\mathbb{F} / \mathcal{O}$ by the multiplicative action of $\mu_{+}$. The volume of $\mathbb{F} / \mathcal{O} \mu_{+}$is 
$2^{-r_{2}}\left|\mu_{+}\right|^{-1} \sqrt{|D|}$, and so we have

$$
\begin{aligned}
\left\langle E(s, m, \cdot) F_{k}, F_{k}\right\rangle=\left|a_{f}(1)\right|^{2} 2^{-r_{2}} \mid \mu_{+} & \left.\right|^{-1} \sqrt{|D|} \int_{\mathbb{R}_{+}^{r} / \mathcal{O}_{+}^{\times}} N y^{s} \lambda_{m}(y) \\
& \times \sum_{\xi>0}\left|\lambda_{\pi}(\xi)\right|^{2} N \xi^{-1}\left|\mathbf{K}_{k}(\xi \kappa y)\right|^{2} N y^{-1} d y^{\times} .
\end{aligned}
$$

Making a change of variable and unfolding the integral over $\mathcal{O}_{+}^{\times}$, this becomes

$$
\begin{aligned}
&\left\langle E(s, m, \cdot) F_{k}, F_{k}\right\rangle=\left|a_{f}(1)\right|^{2} 2^{-r_{2}} \sqrt{|D|} N \kappa \sum_{(\xi)}\left|\lambda_{\pi}(\xi)\right|^{2}(N \xi \kappa)^{-s} \lambda_{-m}(\xi \kappa) \\
& \times \int_{\mathbb{R}_{+}^{r}} N y^{s} \lambda_{m}(y)\left|\mathbf{K}_{k}(y)\right|^{2} N y^{-1} d y^{\times} \\
&=\left|a_{f}(1)\right|^{2} 2^{-r_{2}} \sqrt{|D|} N \kappa^{1-s} \lambda_{-m}(\kappa) \frac{L\left(s, \pi \otimes \pi \otimes \lambda_{-m}\right)}{L\left(2 s, \lambda_{-2 m}\right)} \\
& \times \int_{\mathbb{R}_{+}^{r}} N y^{s} \lambda_{m}(y)\left|\mathbf{K}_{k}(y)\right|^{2} N y^{-1} d y^{\times} .
\end{aligned}
$$

(Note that the factor of $\left|\mu_{+}\right|^{-1}$ vanished because $\mathcal{O}^{+} / \mathcal{O}_{+}^{\times}$counts every ideal with this multiplicity.) We factorise the integral occurring here and pair each factor with the corresponding term of $\left|a_{f}(1)\right|^{2}$ so that

$$
\left\langle E(1 / 2+i t, m, \cdot) F_{k}, F_{k}\right\rangle=C \frac{L\left(1 / 2+i t, \pi \otimes \pi \otimes \lambda_{-m}\right)}{L\left(1, \mathrm{sym}^{2} \pi\right) L\left(1+2 i t, \lambda_{-2 m}\right)} \prod_{j=1}^{r} \mathcal{T}_{j},
$$

where for $j \leq r_{1}$ we have

$$
\mathcal{T}_{j}=\frac{(4 \pi)^{k_{j}}}{\Gamma\left(k_{j}\right)} \int_{0}^{\infty} y^{1 / 2+i t+\beta(m, j)}\left|\mathbf{K}_{j}(y)\right|^{2} y^{-1} d y^{\times},
$$

and for $j>r_{1}$,

$$
\mathcal{T}_{j}=\frac{(2 \pi)^{k_{j}}}{\Gamma\left(k_{j} / 2+1\right)^{2}} \int_{0}^{\infty} y^{1+2 i t+\beta(m, j)}\left|\mathbf{K}_{j}(y)\right|^{2} y^{-2} d y^{\times} .
$$

The integral at real places may be easily calculated to be

$$
\mathcal{T}_{j}=(4 \pi)^{1 / 2-i t-\beta(m, j)} \frac{\Gamma\left(k_{j}-1 / 2+i t+\beta(m, j)\right)}{\Gamma\left(k_{j}\right)},
$$

and the integral at complex places was calculated in 24 to have absolute value

$$
\mathcal{T}_{j}=\frac{\left|\Gamma\left(1 / 2+i t+\beta(m, j)+k_{j} / 2\right) \Gamma(1 / 2+i t+\beta(m, j))\right|^{2}}{\Gamma\left(1+k_{j} / 2\right)^{2}|\Gamma(1+2 i t+2 \beta(m, j))|} .
$$

Both of these terms agree in absolute value with the ratio of gamma factors at the corresponding infinite place of the $L$-functions appearing in (81), which proves formula (79). To prove (80), we use Stirling together with the bound $|\Gamma(\sigma+i t)| \leq$ $\Gamma(\sigma)$ to show that $\left|\mathcal{T}_{j}\right| \ll k_{j}^{-1 / 2}$ for $\nu_{j}$ real and $\mathcal{T}_{j} \ll k_{j}^{-1}(1+|t+\beta(m, j)|)^{-1 / 2} \leq k_{j}^{-1}$ 
for $\nu_{j}$ complex. This gives

$$
\begin{aligned}
\left|\left\langle E(1 / 2+i t, m, \cdot) F_{k}, F_{k}\right\rangle\right| & \ll\left|N k^{-1 / 2} \frac{L\left(1 / 2+i t, \pi \otimes \pi \otimes \lambda_{-m}\right)}{L\left(1, \mathrm{sym}^{2} \pi\right) L\left(1+2 i t, \lambda_{-2 m}\right)}\right| \\
& =N k^{-1 / 2}\left|\frac{L\left(1 / 2+i t, \operatorname{sym}^{2} \pi \otimes \lambda_{-m}\right) L\left(1 / 2+i t, \lambda_{-m}\right)}{L\left(1, \operatorname{sym}^{2} \pi\right) L\left(1+2 i t, \lambda_{-2 m}\right)}\right|,
\end{aligned}
$$

and applying the convex bound $L\left(1 / 2+i t, \lambda_{-m}\right) \ll(1+|t|+\|m\|)^{n / 4+\epsilon}$ and the lower bound $L\left(1+2 i t, \lambda_{-2 m}\right) \gg(1+|t|+\|m\|)^{-\epsilon}$ yields (80).

8.2. Weak subconvexity. Having expressed the inner products $\left\langle\phi F_{k}, F_{k}\right\rangle$ in terms of $L$-values, we now prove Theorem 5 by applying the weak subconvexity result of Soundararajan 39] to these values. This is a theorem which is valid for any Dirichlet series $L(s, \pi)$ over the rationals satisfying certain conditions, which we now describe. The first of these is that $L(s, \pi)$ may be given by an Euler product

$$
L(s, \pi)=\sum_{n=1}^{\infty} \frac{a_{\pi}(n)}{n^{s}}=\prod_{p} \prod_{j=1}^{m}\left(1-\frac{\alpha_{j, \pi}(p)}{p^{s}}\right)^{-1},
$$

and that both the series and product are absolutely convergent for $\operatorname{Re}(s)>1$ (the notation $L(s, \pi)$ is meant to suggest that $\pi$ corresponds to an automorphic representation, although this is not assumed). The second is that there is an Archimedean component

$$
L_{\infty}(s, \pi)=N^{s / 2} \prod_{j=1}^{m} \Gamma_{\mathbb{R}}\left(s+\mu_{j}\right)
$$

for $N \in \mathbb{Z}$ and $\mu_{j} \in \mathbb{C}$, such that the completed $L$-function $\Lambda(s, \pi)=L_{\infty}(s, \pi) L(s, \pi)$ has an analytic continuation to the entire complex plane. Moreover, it should satisfy a functional equation

$$
\Lambda(s, \pi)=\kappa \Lambda(1-s, \tilde{\pi}),
$$

for $\kappa$ a complex number of absolute value one and where

$$
L(s, \widetilde{\pi})=\sum_{n=1}^{\infty} \frac{\overline{a_{\pi}(n)}}{n^{s}} \quad \text { and } \quad L_{\infty}(s, \widetilde{\pi})=N^{s / 2} \prod_{j=1}^{m} \Gamma_{\mathbb{R}}\left(s+\overline{\mu_{j}}\right) .
$$

These conditions are quite general and hold for all the $L$-functions appearing in our triple product identities. In addition, we require some bounds towards the Ramanujan-Selberg conjectures for $\pi$, which predicts that $\left|\alpha_{j, \pi}(p)\right| \leq 1$ and $\operatorname{Re}\left(\mu_{j}\right) \geq 0$. Write

$$
-\frac{L^{\prime}}{L}(s, \pi)=\sum_{n=1}^{\infty} \frac{\lambda_{\pi}(n) \Lambda(n)}{n^{s}},
$$

where $\lambda_{\pi}(n)=0$ unless $n=p^{k}$ is a prime power, in which case it equals $\sum_{j=1}^{m} \alpha_{j, \pi}(p)^{k}$. We require the existence of two constants $A_{0}, A \geq 1$ such that for all $x \geq 1$ the inequality

$$
\sum_{x<n<e x} \frac{\left|\lambda_{\pi}(n)\right|^{2}}{n} \Lambda(n) \leq A^{2}+\frac{A_{0}}{\log e x}
$$

is satisfied; note that the Ramanujan conjecture would imply this with $A=m$ and $A_{0} \ll m^{2}$. The condition on the parameters $\mu_{j}$ is that $\operatorname{Re}\left(\mu_{j}\right) \geq-1+\delta_{m}$ for some 
$\delta_{m}>0$ and all $j$. If we define the analytic conductor of $\pi$ to be

$$
C(\pi)=N \prod_{j=1}^{m}\left(1+\left|\mu_{j}\right|\right),
$$

Soundararajan proves the following.

Theorem 16. Under the assumptions on L stated above,

$$
L(1 / 2, \pi) \ll \frac{C(\pi)^{1 / 4}}{(\log C(\pi))^{1-\epsilon}},
$$

where the implied constant depends on $m, \epsilon, A_{0}, A$ and $\delta_{m}$.

We may prove Theorem 5 by applying this result to the $L$-values $L\left(1 / 2, \operatorname{sym}^{2} \pi \otimes\right.$ $\left.\pi^{\prime}\right)$ and $L\left(1 / 2+i t, \operatorname{sym}^{2} \pi \otimes \lambda_{-m}\right)$ appearing in equations (75) and (80), once we have established that the $L$-functions satisfy the necessary hypotheses. While they are $L$-functions over $F$, they may be considered as being over $\mathbb{Q}$ by formal base change. We begin with $L\left(s, \operatorname{sym}^{2} \pi \otimes \lambda_{-m}\right)$; if $L(s, \pi)$ has the Euler product expansion

$$
L(s, \pi)=\prod_{\mathfrak{p}}\left(1-\frac{\alpha_{\pi}(\mathfrak{p})}{N \mathfrak{p}^{s}}\right)^{-1}\left(1-\frac{\beta_{\pi}(\mathfrak{p})}{N \mathfrak{p}^{s}}\right)^{-1},
$$

then $L\left(s, \operatorname{sym}^{2} \pi \otimes \lambda_{-m}\right)$ is given by the Euler product

$$
\begin{aligned}
L\left(s, \operatorname{sym}^{2} \pi \otimes \lambda_{-m}\right)=\prod_{\mathfrak{p}}(1- & \left.\frac{\alpha_{\pi}(\mathfrak{p})^{2} \lambda_{-m}(\mathfrak{p})}{N \mathfrak{p}^{s}}\right)^{-1} \\
& \quad \times\left(1-\frac{\lambda_{-m}(\mathfrak{p})}{N \mathfrak{p}^{s}}\right)^{-1}\left(1-\frac{\beta_{\pi}(\mathfrak{p})^{2} \lambda_{-m}(\mathfrak{p})}{N \mathfrak{p}^{s}}\right)^{-1} .
\end{aligned}
$$

We have assumed that $\left|\alpha_{\pi}(\mathfrak{p})\right|=\left|\beta_{\pi}(\mathfrak{p})\right|=1$. We recall the definitions of the local zeta functions of $\mathbb{R}$ and $\mathbb{C}$,

$$
\Gamma_{\mathbb{R}}(s)=\pi^{-s / 2} \Gamma(s / 2), \quad \Gamma_{\mathbb{C}}=(2 \pi)^{-s} \Gamma(s) .
$$

The Archimedean factor of $L\left(s, \operatorname{sym}^{2} \pi \otimes \lambda_{-m}\right)$ is

$$
L_{\infty}\left(s, \operatorname{sym}^{2} \pi \otimes \lambda_{-m}\right)=\prod_{j} L_{\infty, j}\left(s, \operatorname{sym}^{2} \pi \otimes \lambda_{-m}\right),
$$

where

$L_{\infty, j}\left(s, \operatorname{sym}^{2} \pi \otimes \lambda_{-m}\right)=\Gamma_{\mathbb{R}}(s+\beta(m, j)+1) \Gamma_{\mathbb{R}}\left(s+\beta(m, j)+k_{j}-1\right) \Gamma_{\mathbb{R}}\left(s+\beta(m, j)+k_{j}\right)$

for $\nu_{j}$ real and

$$
\begin{aligned}
L_{\infty, j}\left(s, \operatorname{sym}^{2} \pi \otimes \lambda_{-m}\right)= & \Gamma_{\mathbb{C}}\left(s+k_{j} / 2+\beta(m, j)\right)^{2} \Gamma_{\mathbb{C}}(s+\beta(m, j)) \\
= & \Gamma_{\mathbb{R}}\left(s+k_{j} / 2+\beta(m, j)\right)^{2} \Gamma_{\mathbb{R}}\left(s+k_{j} / 2+\beta(m, j)+1\right)^{2} \\
& \times \Gamma_{\mathbb{R}}(s+\beta(m, j)) \Gamma_{\mathbb{R}}(s+\beta(m, j)+1)
\end{aligned}
$$

for $\nu_{j}$ complex. In particular, it can be seen that all $\mu_{j}$ satisfy $\operatorname{Re}\left(\mu_{j}\right) \geq 0$. By the work of Gelbart and Jacquet [9], it is known that the completed $L$-function $\Lambda\left(s, \operatorname{sym}^{2} \pi \otimes \lambda_{-m}\right)$ admits an analytic continuation to the whole complex plane and satisfies the functional equation

$$
\Lambda\left(s, \operatorname{sym}^{2} \pi \otimes \lambda_{-m}\right)=\Lambda\left(1-s, \operatorname{sym}^{2} \pi \otimes \lambda_{m}\right) .
$$


The $L$-function we consider therefore satisfies all the hypotheses of Soundararajan's theorem as an Euler product over $F$, and it will continue to do so when considered as a product over $\mathbb{Q}$; in particular, it will continue to satisfy the Ramanujan bound. To apply the theorem to the value $L\left(1 / 2+i t, \operatorname{sym}^{2} \pi \otimes \lambda_{-m}\right)$ we replace $L\left(s, \operatorname{sym}^{2} \pi \otimes\right.$ $\left.\lambda_{-m}\right)$ with the shifted function $L\left(s+i t, \operatorname{sym}^{2} \pi \otimes \lambda_{-m}\right)$, which still satisfies all the hypotheses and whose analytic conductor is now $\ll N k^{2}(1+|t|+\|m\|)^{3 n}$, to obtain

$$
L\left(1 / 2+i t, \operatorname{sym}^{2} \pi \otimes \lambda_{-m}\right) \ll \frac{N k^{1 / 2}(1+|t|+\|m\|)^{3 n / 4}}{(\log N k)^{1-\epsilon}} .
$$

Turning now to $L\left(1 / 2, \operatorname{sym}^{2} \pi \otimes \pi^{\prime}\right)$, let $L\left(s, \pi^{\prime}\right)$ have the Euler product

$$
L\left(s, \pi^{\prime}\right)=\prod_{\mathfrak{p}}\left(1-\frac{\alpha_{\pi}^{\prime}(\mathfrak{p})}{N \mathfrak{p}^{s}}\right)^{-1}\left(1-\frac{\beta_{\pi}^{\prime}(\mathfrak{p})}{N \mathfrak{p}^{s}}\right)^{-1}
$$

so that $L\left(s, \operatorname{sym}^{2} \pi \otimes \pi^{\prime}\right)$ has the product expansion

$$
\begin{aligned}
L\left(s, \operatorname{sym}^{2} \pi \otimes\right. & \left.\pi^{\prime}\right)=\prod_{\mathfrak{p}}\left(1-\frac{\alpha_{\pi}(\mathfrak{p})^{2} \alpha_{\pi}^{\prime}(\mathfrak{p})}{N \mathfrak{p}^{s}}\right)^{-1}\left(1-\frac{\alpha_{\pi}^{\prime}(\mathfrak{p})}{N \mathfrak{p}^{s}}\right)^{-1}\left(1-\frac{\beta_{\pi}(\mathfrak{p})^{2} \alpha_{\pi}^{\prime}(\mathfrak{p})}{N \mathfrak{p}^{s}}\right)^{-1} \\
& \times\left(1-\frac{\alpha_{\pi}(\mathfrak{p})^{2} \beta_{\pi}^{\prime}(\mathfrak{p})}{N \mathfrak{p}^{s}}\right)^{-1}\left(1-\frac{\beta_{\pi}^{\prime}(\mathfrak{p})}{N \mathfrak{p}^{s}}\right)^{-1}\left(1-\frac{\beta_{\pi}(\mathfrak{p})^{2} \beta_{\pi}^{\prime}(\mathfrak{p})}{N \mathfrak{p}^{s}}\right)^{-1} .
\end{aligned}
$$

This $L$-function does not necessarily satisfy the Ramanujan bound because we are not assuming it for the representation $\pi^{\prime}$; however, because $\pi^{\prime}$ is fixed we may still prove the weaker estimate (82) by applying Rankin-Selberg theory to $\pi^{\prime}$.

The Archimedean factor $L_{\infty, j}\left(s, \operatorname{sym}^{2} \pi \otimes \pi^{\prime}\right)$ at a real place is

$$
\begin{aligned}
& L_{\infty, j}\left(s, \operatorname{sym}^{2} \pi \otimes \pi^{\prime}\right) \\
& \quad=\prod_{\varepsilon= \pm 1} \Gamma_{\mathbb{R}}\left(s+k_{j}-1+\varepsilon i r_{j}^{\prime}\right) \Gamma_{\mathbb{R}}\left(s+k_{j}+\varepsilon_{j} r_{j}^{\prime}\right) \Gamma_{\mathbb{R}}\left(s+\varepsilon i r_{j}^{\prime}\right) \Gamma_{\mathbb{R}}\left(s+1+\varepsilon i r_{j}^{\prime}\right),
\end{aligned}
$$

and at a complex place is

$$
\begin{aligned}
& L_{\infty, j}\left(s, \operatorname{sym}^{2} \pi \otimes \pi^{\prime}\right)= \prod_{\varepsilon= \pm 1} \Gamma_{\mathbb{C}}\left(s+k_{j} / 2+\varepsilon i r_{j}^{\prime} / 2\right)^{2} \Gamma_{\mathbb{C}}\left(s+\varepsilon i r_{j}^{\prime} / 2\right) \\
&= \prod_{\varepsilon= \pm 1} \Gamma_{\mathbb{R}}\left(s+k_{j} / 2+\varepsilon i r_{j}^{\prime} / 2\right)^{2} \Gamma_{\mathbb{R}}\left(s+k_{j} / 2+1+\varepsilon i r_{j}^{\prime} / 2\right)^{2} \\
& \times \Gamma_{\mathbb{R}}\left(s+\varepsilon i r_{j}^{\prime} / 2\right) \Gamma_{\mathbb{R}}\left(s+1+\varepsilon i r_{j}^{\prime} / 2\right) .
\end{aligned}
$$

The required bound $\operatorname{Re}\left(\mu_{j}\right) \geq-1+\delta$ now follows from the trivial bounds $\operatorname{Im}\left(r_{j}^{\prime}\right) \leq$ $1 / 2$ for $\nu_{j}$ real and $\operatorname{Im}\left(r_{j}^{\prime}\right) \leq 1$ for $\nu_{j}$ complex. It is known by the work of Garrett [8] that the completed $L$-function is entire in $\mathbb{C}$, and its value at $s$ is equal to its value at $1-s$. The remaining condition on $L\left(s, \operatorname{sym}^{2} \pi \otimes \pi^{\prime}\right)$ is provided by the following lemma.

Lemma 17. $L\left(s, \mathrm{sym}^{2} \pi \otimes \pi^{\prime}\right)$ satisfies the weak Ramanujan bound (82) as a Dirichlet series over $\mathbb{Q}$.

Proof. We have

$$
-\frac{L^{\prime}}{L}\left(s, \operatorname{sym}^{2} \pi \otimes \pi^{\prime}\right)=\sum_{\mathfrak{p}} \log N \mathfrak{p} \sum_{n=1}^{\infty} \frac{\left(\alpha_{\pi}^{\prime n}(\mathfrak{p})+\beta_{\pi}^{\prime n}(\mathfrak{p})\right)\left(\alpha_{\pi}^{2 n}(\mathfrak{p})+1+\beta_{\pi}^{2 n}(\mathfrak{p})\right)}{N \mathfrak{p}^{n s}}
$$


and $L$ satisfying the weak Ramanujan bound as a Dirichlet series over $\mathbb{Q}$ is equivalent to the bound

$$
\sum_{\substack{\mathfrak{p}, n \\ x<N \mathfrak{p}^{n} \leq e x}} \log N \mathfrak{p} \frac{\left|\left(\alpha_{\pi}^{\prime n}(\mathfrak{p})+\beta_{\pi}^{\prime n}(\mathfrak{p})\right)\left(\alpha_{\pi}^{2 n}(\mathfrak{p})+1+\beta_{\pi}^{2 n}(\mathfrak{p})\right)\right|^{2}}{N \mathfrak{p}^{n}} \leq A^{2}+\frac{A_{0}}{\log (e x)} .
$$

After applying the Ramanujan bound $\left|\alpha_{\pi}(\mathfrak{p})\right|=\left|\beta_{\pi}(\mathfrak{p})\right|=1$, we only need to show that

$$
\sum_{\substack{\mathfrak{p}, n \\ x<N \mathfrak{p}^{n} \leq e x}} \log N \mathfrak{p} \frac{\left|\alpha_{\pi}^{\prime n}(\mathfrak{p})+\beta_{\pi}^{\prime n}(\mathfrak{p})\right|^{2}}{N \mathfrak{p}^{n}} \leq A^{2}+\frac{A_{0}}{\log (e x)}
$$

for all $x \geq 1$, where $A$ and $A_{0}$ are constants which are allowed to depend on $\pi^{\prime}$. This follows from Rankin-Selberg theory for $L\left(s, \pi^{\prime} \times \tilde{\pi}^{\prime}\right)$, whose logarithmic derivative is

$$
-\frac{L^{\prime}}{L}\left(s, \pi^{\prime} \times \widetilde{\pi}^{\prime}\right)=\sum_{\mathfrak{p}} \sum_{n=1}^{\infty} \log N \mathfrak{p} \frac{\left|\alpha_{\pi}^{\prime n}(\mathfrak{p})+\beta_{\pi}^{\prime n}(\mathfrak{p})\right|^{2}}{N \mathfrak{p}^{n s}} .
$$

Because $L\left(s, \pi^{\prime} \times \widetilde{\pi}^{\prime}\right)$ has a classical zero-free region $\operatorname{Re}(s) \geq 1-c_{\pi}^{\prime} / \log (1+|t|)$, it follows in the same way as the proof of the prime number theorem that

$$
\sum_{\substack{\mathfrak{p}, n \\ x<N \mathfrak{p}^{n} \leq e x}} \frac{\log N \mathfrak{p}\left|\alpha_{\pi}^{\prime n}(\mathfrak{p})+\beta_{\pi}^{\prime n}(\mathfrak{p})\right|^{2}}{N \mathfrak{p}^{n s}}=1+O_{\pi^{\prime}}\left(\frac{1}{\log (e x)}\right),
$$

from which (82) follows.

As $L\left(s, \operatorname{sym}^{2} \pi \otimes \pi^{\prime}\right)$ has analytic conductor $\ll N k^{4}$, we may now apply the weak subconvex estimate to $L\left(1 / 2, \mathrm{sym}^{2} \pi \otimes \pi^{\prime}\right)$ to complete the proof of Theorem 5 .

\section{Conclusion of Proof}

We now conclude the proof of Theorem 1 by presenting the way in which Theorems 4 and 5 may be combined as in Holowinsky and Soundararajan's paper [16]. This relies on a lower bound for $L\left(1, \mathrm{sym}^{2} \pi\right)$ and a relation between this value and the quantity $M_{k}(\pi)$ appearing in Theorem 4 .

We first consider the symmetric square $L$-function $L\left(s, \mathrm{sym}^{2} \pi\right)$, whose definition and basic analytic properites were given in section 8.2, and collect some important results on it due to work of Gelbart and Jacquet [9, Hoffstein and Lockhart [13, Goldfeld, Hoffstein and Lieman [10, and Blomer and Harcos [4]. The lower bound we shall use for $L\left(1, \operatorname{sym}^{2} \pi\right)$ is proved using the symmetric square lift of Gelbart and Jacquet 9 from $G L(2)$ to $G L(3)$, which shows that $L\left(s, \operatorname{sym}^{2} \pi\right)$ is the standard $L$-function of a cuspidal automorphic form on $G L(3)$. Using the Rankin-Selberg convolution for this form, one may then establish a standard zero-free region for $L\left(s, \operatorname{sym}^{2} \pi\right)$. For instance, using Theorem 5.42 (or Theorem 5.44) of Iwaniec and Kowalski [18] one may show that for some constant $c>0$ the region

$$
\mathcal{R}=\left\{s=\sigma+i t: \sigma \geq 1-\frac{c}{\log \|k\|(1+|t|)}\right\}
$$

contains no zero of $L\left(s, \mathrm{sym}^{2} \pi\right)$ other than possibly a simple real zero. This exceptional zero is ruled out by work of Hoffstein and Lockhart [13] (see the appendix by Goldfeld, Hoffstein and Lieman [10] for the case $F=\mathbb{Q}$, and the generalisation to a number field in section 2.9 of the article of Blomer and Harcos [4]), who show 
that there is an effectively computable choice of $c>0$ such that $\mathcal{R}$ is totally zero free. Furthermore, it is shown in [10] and [4] that

$$
L\left(1, \operatorname{sym}^{2} \pi\right) \gg \frac{1}{\log \|k\|} .
$$

The first consequence of this lower bound is the following.

Lemma 18. The quantity $R_{k}(\pi)$ appearing in Theorem 4 satisfies

$$
R_{k}(\pi) \ll \frac{(\log \|k\|)^{\epsilon}}{(\log \|k\|) L\left(1, \operatorname{sym}^{2} \pi\right)} \ll(\log \|k\|)^{\epsilon} .
$$

Proof. By applying the weak subconvex bound of section 8.2 to the $L$-function $L\left(s, \operatorname{sym}^{2} \pi \otimes \lambda_{m}\right)$, we have

$$
\left|L\left(1 / 2+i t, \operatorname{sym}^{2} \pi \otimes \lambda_{m}\right)\right| \ll \frac{N k^{1 / 2}(1+|t|+\|m\|)^{3 n / 4}}{(\log \|k\|)^{1-\epsilon}}
$$

for any $t \in \mathbb{R}$ and $m \in \mathbb{Z}^{r-1}$. The lemma follows immediately by substituting this in the formula for $R_{k}(\pi)$ and applying the lower bound (83) for $L\left(1, \mathrm{sym}^{2} \pi\right)$.

The relationship between $M_{k}(\pi)$ and $L\left(1, \operatorname{sym}^{2} \pi\right)$ we shall use is based on the following lemma.

Lemma 19. We have

$$
L\left(1, \operatorname{sym}^{2} \pi\right) \gg(\log \log \|k\|)^{-3} \exp \left(\sum_{N \mathfrak{p} \leq\|k\|} \frac{\lambda_{\pi}\left(\mathfrak{p}^{2}\right)}{N \mathfrak{p}}\right) .
$$

The proof of this over $\mathbb{Q}$ in 16 may be extended to a number field; the only modification is generalising the asymptotic $\sum_{p \leq x} 1 / p=\log \log x+O(1)$ to $\sum_{N \mathfrak{p} \leq x} 1 / N \mathfrak{p}$ $=\log \log x+O(1)$. Lemma 19 gives us the required estimate for $M_{k}(\pi)$ below.

Lemma 20. We have

$$
M_{k}(\pi) \ll(\log \|k\|)^{1 / 6}(\log \log \|k\|)^{9 / 2} L\left(1, \operatorname{sym}^{2} \pi\right)^{1 / 2} .
$$

Proof. From the inequality $2|x| \leq \frac{2}{3}+\frac{3}{2} x^{2}$ and the Hecke relations, we obtain

$$
\begin{aligned}
2 \sum_{N \mathfrak{p} \leq\|k\|} \frac{\left|\lambda_{\pi}(\mathfrak{p})\right|}{N \mathfrak{p}} & \leq \frac{2}{3} \sum_{N \mathfrak{p} \leq\|k\|} \frac{1}{N \mathfrak{p}}+\frac{3}{2} \sum_{N \mathfrak{p} \leq\|k\|} \frac{\lambda_{\pi}(\mathfrak{p})^{2}}{N \mathfrak{p}} \\
& =\frac{13}{6} \sum_{N \mathfrak{p} \leq\|k\|} \frac{1}{N \mathfrak{p}}+\frac{3}{2} \sum_{N \mathfrak{p} \leq\|k\|} \frac{\lambda_{\pi}\left(\mathfrak{p}^{2}\right)}{N \mathfrak{p}} .
\end{aligned}
$$

Using Lemma 19 and $\sum_{N \mathfrak{p} \leq x} 1 / N \mathfrak{p}=\log \log x+O(1)$, the lemma follows.

We may now prove the decay of $\left\langle\phi F_{k}, F_{k}\right\rangle$ for $\phi$ a Hecke-Maass cusp form or pure incomplete Eisenstein series. We consider the Maass case first. If $L\left(1, \operatorname{sym}^{2} \pi\right) \geq$ $(\log \|k\|)^{-7 / 15}$, then Theorem 5 gives $\left\langle\phi F_{k}, F_{k}\right\rangle \ll(\log \|k\|)^{-1 / 30+\epsilon}$. Otherwise, from Lemma 20 we have that $M_{k}(\pi) \ll(\log \|k\|)^{-1 / 15+\epsilon}$, and now Theorem 4 gives $\left\langle\phi F_{k}, F_{k}\right\rangle \ll(\log \|k\|)^{-1 / 30+\epsilon}$. The bound of Theorem 1 therefore holds in either case. 
In the Eisenstein case, we begin by showing how Theorem 5 may be used to bound $\left\langle\phi F_{k}, F_{k}\right\rangle$ in the cases where $L\left(1, \operatorname{sym}^{2} \pi\right)$ is large. By Mellin inversion, we may write

$$
E(\psi, m \mid z)=\frac{1}{2 \pi i} \int_{(\sigma)} \Psi(-s) E(s, m, z) d s,
$$

where $\Psi$ is the Mellin transform of $\psi$. We may move the line of integration to $\sigma=1 / 2$ to obtain

$$
E(\psi, m \mid z)=\frac{1}{\operatorname{Vol}(Y)}\langle E(\psi, m \mid z), 1\rangle+\frac{1}{2 \pi i} \int_{(1 / 2)} \Psi(-s) E(s, m, z) d s,
$$

and so

$\left\langle E(\psi, m \mid z) F_{k}, F_{k}\right\rangle=\frac{1}{\operatorname{Vol}(Y)}\langle E(\psi, m \mid z), 1\rangle+\frac{1}{2 \pi i} \int_{(1 / 2)} \Psi(-s)\left\langle E(s, m, z) F_{k}, F_{k}\right\rangle d s$.

We now apply Theorem 5 to obtain the bound

$$
\begin{aligned}
\int_{(1 / 2)} \Psi(-s)\left\langle E(s, m, z) F_{k}, F_{k}\right\rangle d s & \ll \int_{-\infty}^{\infty}|\Psi(-1 / 2-i t)| \frac{(1+|t|+\|m\|)^{2 n}}{(\log \|k\|)^{1-\epsilon} L\left(1, \operatorname{sym}^{2} \pi\right)} d t \\
& \ll \frac{(\log \|k\|)^{-1+\epsilon}}{L\left(1, \operatorname{sym}^{2} \pi\right)} .
\end{aligned}
$$

It follows by substituting this in (84) that

$$
\left|\left\langle E(\psi, m \mid z) F_{k}, F_{k}\right\rangle-\frac{1}{\operatorname{Vol}(Y)}\langle E(\psi, m \mid z), 1\rangle\right| \ll \frac{(\log \|k\|)^{-1+\epsilon}}{L\left(1, \operatorname{sym}^{2} \pi\right)} .
$$

Therefore if $L\left(1, \operatorname{sym}^{2} \pi\right) \geq(\log \|k\|)^{-13 / 15}$, we obtain the bound of Theorem 1. If $L\left(1, \operatorname{sym}^{2} \pi\right)<(\log \|k\|)^{-13 / 15}$, Lemma 20 gives $M_{k}(\pi) \ll(\log \|k\|)^{-4 / 15+\epsilon}$. Applying Theorem 4 with the bound on $R_{k}(\pi)$ provided by Lemma 18, we have

$$
\begin{aligned}
\left|\left\langle E(\psi, m \mid z) F_{k}, F_{k}\right\rangle-\frac{1}{\operatorname{Vol}(Y)}\langle E(\psi, m \mid z), 1\rangle\right| & \ll(\log \|k\|)^{\epsilon} M_{k}(\pi)^{1 / 2} \\
& \ll(\log \|k\|)^{-2 / 15+\epsilon},
\end{aligned}
$$

and so the bound of Theorem 1 holds in this case also.

\section{Equidistribution OF ZERO CURRENTS}

This section contains the proof of Theorem 6 on the equidistribution of the zero divisors of holomorphic Hecke modular forms. The proof is based on ideas from complex potential theory, which may be described in the general context of a compact complex manifold $M$ with a positive holomorphic line bundle $L$. Suppose that $L$ has been equipped with a Hermitian metric $h$, and let $\omega=c_{1}(h)$ be the associated Kähler form. If $s_{T} \in H^{0}\left(M, L^{T}\right)$ are a sequence of $L^{2}$ normalised sections of $L^{T}$ whose mass becomes equidistributed on $M$, potential theory may be used to show that their normalised zero divisors $\frac{1}{T} Z_{T}$ tend weakly to $\omega$ in the sense of currents described in section 3.1. This was first discovered by Nonnenmacher and Vorros 30 in the context of quantum maps on tori, and extended in the generality described here by Shiffman and Zelditch [35]. If we now let $\mathbb{H}^{n}$ denote the product of $n$ upper half planes and let $L_{k}$ be the line bundle of differentials of the form $f(z) \otimes_{i} d z_{i}^{k_{i} / 2}$ on $\mathbb{H}^{n}$, or its quotient by $\Gamma$, theorem $[6$ is thus an extension of the result in 35] to the bundle $L_{k}$ over the noncompact manifold $Y$. We shall prove it 
by applying the methods of Shiffman and Zelditch, and adding the adjustments of Rudnick 31] to deal with the cusp.

We may give $L_{k}$ the natural Hermitian inner product $\left\|\otimes d z_{i}^{k_{i} / 2}\right\|^{2}=y^{k}$, whose associated Kähler form $\omega$ is

$$
\begin{aligned}
\omega & =\frac{-i}{2 \pi} \partial \bar{\partial} \log y^{k} \\
& =\frac{1}{4 \pi} \sum_{t=1}^{n} k_{t} y_{t}^{-2} d x_{t} \wedge d y_{t} .
\end{aligned}
$$

If $f$ is a holomorphic modular form of weight $k, f \otimes d z_{i}^{k_{i} / 2}$ is then a section of $L_{k}$ with $\left\|f \otimes d z_{i}^{k_{i} / 2}\right\|^{2}=|f(z)|^{2} y^{k}$. We let $Z_{f}$ be the zero divisor of $f$ on $Y$, and $\widetilde{Z}_{f}$ its pullback to $\mathbb{H}^{n}$. For $\phi \in A^{(n-1, n-1)}\left(\mathbb{H}^{n}\right)$ smooth and compactly supported, let

$$
F_{\phi}=\sum_{\gamma \in \Gamma} \gamma^{*} \phi
$$

be its symmetrisation under $\Gamma$. If $\left\{f_{T}\right\}$ is a sequence of modular forms of weight $T k=\left(T k_{i}\right)$ as in Theorem 6, we shall compare $\omega$ with the normalised zero divisors $\frac{1}{T} Z_{T}$ of $f_{T}$ by testing them against the differential forms $F_{\phi}$ using the following lemma.

Lemma 21. If $f$ is a holomorphic modular form of weight $k$ on $Y$, then

$$
\int_{Z_{f}} F_{\phi}=\int_{Y} F_{\phi} \wedge \omega+\frac{i}{\pi} \int_{\mathbb{H}^{n}} \log \left(y^{k / 2}|f(z)|\right) \partial \bar{\partial} \phi .
$$

Proof. By unfolding $F_{\phi}$, we get

$$
\int_{Z_{f}} F_{\phi}=\int_{\widetilde{Z}_{f}} \phi
$$

As $\widetilde{Z}_{f}$ is the zero divisor of the global holomorphic function $f$ on $\mathbb{H}^{n}$, we may apply the Poincaré-Lelong formula to the RHS of (85), obtaining

$$
\begin{aligned}
\int_{Z_{f}} F_{\phi} & =\frac{i}{\pi} \int_{\mathbb{H}^{n}} \log |f(z)| \partial \bar{\partial} \phi \\
& =-\frac{i}{\pi} \int_{\mathbb{H}^{n}} \log y^{k / 2} \partial \bar{\partial} \phi+\frac{i}{\pi} \int_{\mathbb{H}^{n}} \log \left(y^{k / 2}|f(z)|\right) \partial \bar{\partial} \phi
\end{aligned}
$$

After integration by parts the first term becomes

$$
-\frac{i}{\pi} \int_{\mathbb{H}^{n}} \partial \bar{\partial} \log y^{k / 2} \phi=\int_{\mathbb{H}^{n}} \omega \wedge \phi
$$

and may be refolded to $\int_{Y} \omega \wedge F_{\phi}$, which completes the proof.

After applying Lemma 21 to $\frac{1}{T} Z_{T}$, we are left with proving that

$$
\frac{1}{T} \log \left(y^{T k / 2}\left|f_{T}(z)\right|\right) \stackrel{w^{*}}{\longrightarrow} 0
$$

locally everywhere. As in [31, 35, this will follow from the plurisubharmonicity of $\log \left|f_{T}\right|$ and the equidistribution result $y^{T k}\left|f_{T}(z)\right|^{2} \stackrel{w^{*}}{\longrightarrow} c$, once we know that $\frac{1}{T} \log \left(y^{T k / 2}\left|f_{T}(z)\right|\right)$ is bounded above and has $\lim$ sup equal to 0 , and that both properties hold locally uniformly. Both of these are provided by the following lemma and the assumption (which we may clearly make) that the $f_{T}$ are $L^{2}$ normalised. 
Lemma 22. If $f$ is a Hecke cusp form of weight $k$ for $\Gamma$, then we have

$$
\frac{y^{k}|f(z)|^{2}}{\|f\|^{2}} \ll_{\Gamma} N k^{5 / 2+\epsilon}
$$

uniformly for $z$ in compact subsets of $\mathbb{H}^{n}$.

Proof. Assume $\|f\|^{2}=1$. We shall bound $|f|$ using its Fourier expansion

$$
f(z)=\sum_{\xi>0} a_{f}(\xi) e(\operatorname{tr}(\xi \kappa z))
$$

and the proportionality relation $a_{f}(\xi)=\lambda_{\pi}(\xi) a_{f}(1) \xi^{(k-1) / 2}$ with $\lambda_{\pi}(\xi) \ll N \xi^{\epsilon}$. Applying these and the normalisation of $a_{f}(1)$ from (22), we have

$$
\begin{aligned}
y^{k / 2}|f(z)| & \leq \sum_{\xi>0}\left|a_{f}(\xi)\right| y^{k / 2} \exp (-2 \pi \operatorname{tr}(\xi \kappa y)) \\
& \ll \kappa^{k / 2} N k^{\epsilon} \prod_{i=1}^{n} \frac{(4 \pi)^{k_{i} / 2}}{\Gamma\left(k_{i}\right)^{1 / 2}} \sum_{\xi>0} \xi^{(k-1) / 2+\epsilon} y^{k / 2} \exp (-2 \pi \operatorname{tr}(\xi \kappa y)) \\
& =N k^{\epsilon} \prod_{i=1}^{n} \frac{1}{\Gamma\left(k_{i}\right)^{1 / 2}} \sum_{\xi>0} N \xi^{-1 / 2+\epsilon}(4 \pi \xi \kappa y)^{k / 2} \exp (-2 \pi \operatorname{tr}(\xi \kappa y)) \\
& \leq N k^{\epsilon} \prod_{i=1}^{n} \frac{1}{\Gamma\left(k_{i}\right)^{1 / 2}} \sum_{\xi>0}(4 \pi \xi \kappa y)^{k / 2} \exp (-2 \pi \operatorname{tr}(\xi \kappa y)) .
\end{aligned}
$$

We define $g_{i}(x)=x^{k_{i} / 2} e^{-x / 2}$ and let $g: \mathbb{R}_{+}^{n} \rightarrow \mathbb{R}$ be the product function. If we define $L$ to be the semi-lattice $4 \pi \kappa y \mathcal{O} \cap \mathbb{R}_{+}^{n}$, the upper bound above may be written as

$$
y^{k / 2}|f(z)| \ll N k^{\epsilon} \prod_{i=1}^{n} \frac{1}{\Gamma\left(k_{i}\right)^{1 / 2}} \sum_{x \in L} g(x) .
$$

We now apply a lemma bounding the sum in (87) in terms of various integrals of $g$. Suppose $g_{i}(x)$ is increasing for $x<t_{i}$ and decreasing for $x>t_{i}$. Let $\mathcal{P}$ be the set of subsets of $\{1, \ldots, n\}$, and for $S \in \mathcal{P}$ define the subspace $H_{S} \in \mathbb{R}_{+}^{n}$ by

$$
H_{S}=\left\{x \in \mathbb{R}_{+}^{n} \mid x_{i}=t_{i}, i \in S\right\} .
$$

We then have the following bound (whose proof we omit) on $\sum_{x \in L} g(x)$.

\section{Lemma 23.}

$$
\begin{aligned}
\sum_{x \in L} g(x) & \ll \sum_{S \in \mathcal{P}} \int_{H_{S}} g d v \\
& =\prod_{i=1}^{n}\left(\int_{0}^{\infty} g(t) d t+g\left(t_{i}\right)\right),
\end{aligned}
$$

where the implied constant is bounded in compact families of lattices $L$. 
Applying this to (87) gives

$$
\begin{aligned}
y^{k / 2}|f(z)| & \ll N k^{\epsilon} \prod_{i=1}^{n} \frac{1}{\Gamma\left(k_{i}\right)^{1 / 2}}\left(\int_{0}^{\infty} x^{k_{i} / 2} e^{-x / 2} d x+k_{i}^{k_{i} / 2} e^{-k_{i} / 2}\right) \\
& =N k^{\epsilon} \prod_{i=1}^{n} \frac{1}{\Gamma\left(k_{i}\right)^{1 / 2}}\left(2^{k_{i} / 2+1} \Gamma\left(k_{i} / 2+1\right)+k_{i}^{k_{i} / 2} e^{-k_{i} / 2}\right) \\
& \ll N k^{\epsilon} \prod_{i=1}^{n}\left(k_{i}^{5 / 4}+k_{i}^{-3 / 4}\right) \\
& \ll N k^{5 / 4+\epsilon} .
\end{aligned}
$$

The local uniformity of Lemma 23 in $L$ gives the local uniformity of this bound, which completes the proof of Lemma 22 and Theorem 6 .

\section{Appendix}

We include here a number of routine calculations that were omitted during the proof of Propositions 9 and 13. These are the Fourier expansions of Eisenstein series over mixed number fields, the $L^{2}$ normalisations of cohomological automorphic forms, the calculation of the volume form of $\mathbb{H}_{F}$ with respect to the coordinates in the cusp of $Y$, and the verification of the main term picked up in the contour shifts in Lemma 12 and equation (49).

11.1. Fourier expansions of Eisenstein series. We recall the definition of $E(s, m, z)$ for $s \in \mathbb{C}$ and $m \in \mathbb{Z}^{r-1}$,

$$
E(s, m, z)=\sum_{\gamma \in \Gamma_{\infty} \backslash \Gamma} N(y(\gamma z))^{s} \lambda_{m}(y(\gamma z)) .
$$

We let $s_{i}=s+\beta(m, i) / \delta_{i}$, so that this may be rewritten as

$$
E(s, m, z)=\sum_{\gamma \in \Gamma_{\infty} \backslash \Gamma} \prod_{i=1}^{r} y_{i}(\gamma z)^{\delta_{i} s_{i}} .
$$

The map sending $\gamma \in \Gamma_{\infty} \backslash \Gamma$ to its lower two entries is a bijection from $\Gamma_{\infty} \backslash \Gamma$ to the set of pairs $\{c, d\}$ of relatively prime elements of $\mathcal{O}$ modulo $\mathcal{O}^{\times}$, and $y_{i}(\gamma z)$ may be expressed in terms of this pair as

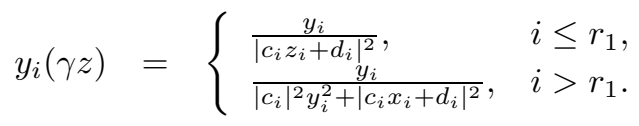

Therefore if we define $F(s, m, z)$ by

$$
F(s, m, z)=\sum_{\{c, d\}} \prod_{i \leq r_{1}} \frac{y_{i}^{s_{i}}}{\left|c_{i} z_{i}+d_{i}\right|^{2 s_{i}}} \prod_{i>r_{1}} \frac{y_{i}^{2 s_{i}}}{\left(\left|c_{i}\right|^{2} y_{i}^{2}+\left|c_{i} x_{i}+d_{i}\right|^{2}\right)^{2 s_{i}}},
$$

where the sum is over all pairs $\{c, d\}$ of elements of $\mathcal{O}$ modulo $\mathcal{O}^{\times}$, we have $F(s, m, z)=\zeta\left(2 s, \lambda_{-2 m}\right) E(s, m, z)$. The $\xi$ th Fourier coefficient of $F(s, m, z)$ is the integral

$$
a_{\xi}(s, m, y)=\frac{2^{r_{2}}}{\sqrt{|D|}} \int_{\mathbb{F} / \mathcal{O}} F(x+j y, s, m) e(-\operatorname{tr}(\xi \kappa x)) d x .
$$


We begin by collecting the terms in (88) with $c=0$ to write

$$
\begin{aligned}
F(s, m, z)=N y^{s} \lambda_{m}(y) \zeta & \left(2 s, \lambda_{-2 m}\right)+\sum_{(c)} \frac{N y^{s} \lambda_{m}(y)}{N c^{2 s} \lambda_{2 m}(c)} \sum_{d \bmod (c)} \sum_{\alpha \in \mathcal{O}} \\
\times & \prod_{i \leq r_{1}} \frac{1}{\left|z_{i}+\frac{d_{i}}{c_{i}}+\alpha_{i}\right|^{2 s_{i}}} \prod_{i>r_{1}} \frac{1}{\left(y_{i}^{2}+\left|x_{i}+\frac{d_{i}}{c_{i}}+\alpha_{i}\right|^{2}\right)^{2 s_{i}}} .
\end{aligned}
$$

Substituting this into (89) and unfolding over $\mathcal{O}$, we express $a_{\xi}(s, m, y)$ as

$$
\begin{aligned}
a_{\xi}(s, m, y)=\delta_{\xi 0} N y^{s} \lambda_{m}(y) & \zeta\left(2 s, \lambda_{-2 m}\right)+\frac{2^{r_{2}}}{\sqrt{|D|}} \sum_{(c)} \frac{N y^{s} \lambda_{m}(y)}{N c^{2 s} \lambda_{2 m}(c)} \sum_{d \bmod (c)} \\
\times & \prod_{i \leq r_{1}} \int_{\mathbb{R}} \frac{e\left(-\xi_{i} \kappa_{i} x_{i}\right) d x_{i}}{\left|z_{i}+\frac{d_{i}}{c_{i}}\right|^{2 s_{i}}} \prod_{i>r_{1}} \int_{\mathbb{C}} \frac{e\left(-\operatorname{tr}\left(\xi_{i} \kappa_{i} x_{i}\right)\right) d x_{i}}{\left(y_{i}^{2}+\left|x_{i}+\frac{d_{i}}{c_{i}}\right|^{2}\right)^{2 s_{i}}},
\end{aligned}
$$

where $d x_{i}$ denotes Lebesgue measure on $\mathbb{C}$. We first consider the case $\xi=0$.

$$
\begin{aligned}
a_{0}(s, m, y)= & N y^{s} \lambda_{m}(y) \zeta\left(2 s, \lambda_{-2 m}\right)+\frac{2^{r_{2}}}{\sqrt{|D|}} \sum_{(c)} \frac{N y^{s} \lambda_{m}(y)}{N c^{2 s} \lambda_{2 m}(c)} \sum_{d \bmod (c)} \\
\times & \prod_{i \leq r_{1}} \int_{\mathbb{R}} \frac{d x_{i}}{\left|z_{i}+\frac{d_{i}}{c_{i}}\right|^{2 s_{i}}} \prod_{i>r_{1}} \int_{\mathbb{C}} \frac{d x_{i}}{\left(y_{i}^{2}+\left|x_{i}+\frac{d_{i}}{c_{i}}\right|^{2}\right)^{2 s_{i}}} \\
= & N y^{s} \lambda_{m}(y) \zeta\left(2 s, \lambda_{-2 m}\right)+\frac{2^{r_{2}}}{\sqrt{|D|}} \sum_{(c)} \frac{N y^{1-s} \lambda_{-m}(y)}{N c^{2 s-1} \lambda_{2 m}(c)} \\
& \times \prod_{i \leq r_{1}} \int_{\mathbb{R}} \frac{d x_{i}}{\left(1+x_{i}^{2}\right)^{s_{i}}} \prod_{i>r_{1}} \int_{\mathbb{C}} \frac{d x_{i}}{\left(1+\left|x_{i}\right|^{2}\right)^{2 s_{i}}} \\
= & N y^{s} \lambda_{m}(y) \zeta\left(2 s, \lambda_{-2 m}\right)+\frac{\pi^{n / 2}}{\sqrt{|D|}} N y^{1-s} \lambda_{-m}(y) \zeta\left(2 s-1, \lambda_{-2 m}\right) \\
& \times \prod_{i \leq r_{1}} \frac{\Gamma(s+\beta(m, i)-1 / 2)}{\Gamma(s+\beta(m, i))} \prod_{i>r_{1}} \frac{2}{2 s+\beta(m, i)-1} .
\end{aligned}
$$

On dividing through by $\zeta\left(2 s, \lambda_{-2 m}\right)$, this agrees with the expression given in section 6.1 .

When $\xi \neq 0$, we have

$$
\begin{aligned}
& a_{\xi}(s, m, y)= \frac{2^{r_{2}}}{\sqrt{|D|}} \sum_{(c)} \frac{N y^{s} \lambda_{m}(y)}{N c^{2 s} \lambda_{2 m}(c)} \sum_{d \bmod (c)} \\
& \times \prod_{i \leq r_{1}} \int_{\mathbb{R}} \frac{e\left(-\xi_{i} \kappa_{i} x_{i}\right) d x_{i}}{\left|z_{i}+\frac{d_{i}}{c_{i}}\right|^{2 s_{i}}} \prod_{i>r_{1}} \int_{\mathbb{C}} \frac{e\left(-\operatorname{tr}\left(\xi_{i} \kappa_{i} x_{i}\right)\right) d x_{i}}{\left(y_{i}^{2}+\left|x_{i}+\frac{d_{i}}{c_{i}}\right|^{2}\right)^{2 s_{i}}} \\
&=\frac{2^{r_{2}}}{\sqrt{|D|}} \sum_{(c)} \frac{N y^{1-s} \lambda_{-m}(y)}{N c^{2 s} \lambda_{2 m}(c)} \sum_{d \bmod (c)} e\left(\operatorname{tr}\left(\frac{\xi \kappa d}{c}\right)\right) \\
& \times \prod_{i \leq r_{1}} \int_{\mathbb{R}} \frac{e\left(-\xi_{i} \kappa_{i} y_{i} x_{i}\right) d x_{i}}{\left(1+x_{i}^{2}\right)^{s_{i}}} \prod_{i>r_{1}} \int_{\mathbb{C}} \frac{e\left(-\operatorname{tr}\left(\xi_{i} \kappa_{i} y_{i} x_{i}\right)\right) d x_{i}}{\left(1+\left|x_{i}\right|^{2}\right)^{2 s_{i}}} .
\end{aligned}
$$


The integral at real places is equal to

$$
\frac{2 \pi^{s_{i}}}{\Gamma\left(s_{i}\right)}\left(\xi_{i} \kappa_{i} y_{i}\right)^{s_{i}-1 / 2} K_{s_{i}-1 / 2}\left(2 \pi\left|\xi_{i} \kappa_{i}\right| y_{i}\right)
$$

and the integral at complex places may be calculated as in section 3 of [33] as follows:

$$
\begin{aligned}
\int_{\mathbb{C}} \frac{e\left(-\operatorname{tr}\left(\xi_{i} \kappa_{i} y_{i} x_{i}\right)\right) d x_{i}}{\left(1+\left|x_{i}\right|^{2}\right)^{2 s_{i}}} & =\int_{0}^{\infty} \int_{0}^{2 \pi} \frac{e\left(-2 y_{i} r\left|\xi_{i} \kappa_{i}\right| \sin (\theta+\alpha)\right)}{\left(r^{2}+1\right)^{2 s_{i}}} r d \theta d r \\
& =\int_{0}^{\infty} \frac{r}{\left(r^{2}+1\right)^{2 s_{i}}} \int_{0}^{2 \pi} e\left(-2 y_{i} r\left|\xi_{i} \kappa_{i}\right| \sin \theta\right) d \theta d r \\
& =\int_{0}^{\infty} \frac{J_{0}\left(4 \pi r\left|\xi_{i} \kappa_{i}\right| y_{i}\right)}{\left(r^{2}+1\right)^{2 s_{i}}} d r \\
& =\frac{\left(4 \pi\left|\xi_{i} \kappa_{i}\right| y_{i}\right)^{2 s_{i}-1}}{\Gamma\left(2 s_{i}\right) 2^{2 s_{i}-1}} K_{2 s_{i}-1}\left(4 \pi\left|\xi_{i} \kappa_{i}\right| y_{i}\right) .
\end{aligned}
$$

(See [11, 6.565 for the evaluation of the final integral.) It follows from (90) and (91) that $a_{\xi}(s, m, y)$ is equal to the product of a collection of Bessel functions and Gamma factors which agree with those appearing in the formula for $E(s, m, z)$ in section 6.1 multiplied by the following constant term and power of $y$ :

$$
\begin{aligned}
\frac{2^{r_{2}}}{\sqrt{|D|}} N y^{1-s} \lambda_{-m}(y) \sum_{(c)} \sum_{d \bmod (c)} & e\left(\operatorname{tr}\left(\frac{\xi \kappa d}{c}\right)\right) \\
& \times \prod_{i \leq r_{1}} 2 \pi^{s_{i}}\left(\xi_{i} \kappa_{i} y_{i}\right)^{s_{i}-1 / 2} \prod_{i>r_{1}} \frac{\left(4 \pi\left|\xi_{i} \kappa_{i}\right| y_{i}\right)^{2 s_{i}-1}}{2^{2 s_{i}-1}} .
\end{aligned}
$$

The power of $y$ simplifies to $\sqrt{N y}$, while the constant may be simplified as

$$
\begin{aligned}
\frac{2^{r_{2}}}{\sqrt{|D|}} \sum_{(c)} \sum_{d \bmod (c)} e\left(\operatorname{tr}\left(\frac{\xi \kappa d}{c}\right)\right) \prod_{i \leq r_{1}} 2 \pi^{s_{i}}\left(\xi_{i} \kappa_{i}\right)^{s_{i}-1 / 2} \prod_{i>r_{1}}\left(2 \pi\left|\xi_{i} \kappa_{i}\right|\right)^{2 s_{i}-1} \\
=\frac{2^{r} \pi^{n s-r_{2}}}{\sqrt{|D|}} \sigma_{1-2 s,-2 m}(\xi \kappa) N(\xi \kappa)^{s-1 / 2} \lambda_{m}(\xi \kappa) \prod_{i>r_{1}} 2^{2 s_{i}-1} \\
=\frac{2^{r} \pi^{n s-r_{2}}}{\sqrt{|D|}} \sigma_{1-2 s,-2 m}(\xi \kappa) N(\delta \xi \kappa)^{s-1 / 2} \lambda_{m}(\delta \xi \kappa) .
\end{aligned}
$$

After dividing through by $\zeta\left(2 s, \lambda_{-2 m}\right)$, we obtain the formula of section 6.1

11.2. $L^{2}$ normalisations. This section contains the calculation of the $L^{2}$ normalisations of the Fourier coefficients of our forms $F_{k}$. The normalisations are based on the equation

$$
\operatorname{Res}_{s=1}\left\langle E(s, z) F_{k}, F_{k}\right\rangle=\left\langle F_{k}, F_{k}\right\rangle \operatorname{Res}_{s=1} \phi(s),
$$

where $E(s, z)=E(s, 0, z)$ and $\phi(s)$ is the scattering coefficient in the constant term. $\operatorname{Res}_{s=1} \phi(s)$ is given by

$$
\begin{aligned}
\operatorname{Res}_{s=1} \phi(s) & =\frac{\pi^{n / 2}}{\sqrt{|D|}} \frac{\operatorname{Res}_{s=1} \zeta_{F}(s)}{2 \zeta_{F}(2)} \prod_{i \leq r_{1}} \frac{\Gamma(1 / 2)}{\Gamma(1)} \prod_{i>r_{1}} 2 \\
& =\frac{2^{r_{2}-1} \pi^{\left(n+r_{1}\right) / 2} \operatorname{Res}_{s=1} \zeta_{F}(s)}{\sqrt{|D|} \zeta_{F}(2)} .
\end{aligned}
$$


We may calculate $\operatorname{Res}_{s=1}\left\langle E(s, z) F_{k}, F_{k}\right\rangle$ by unfolding and compare the result with the RHS of (92):

$$
\begin{aligned}
\left\langle E(s, z) F_{k}, F_{k}\right\rangle & =\int_{\Gamma_{\infty} \backslash \mathbb{H}_{F}} N y^{s}\left|F_{k}\right|^{2} d v \\
& =\left|a_{f}(1)\right|^{2} \frac{\sqrt{|D|}}{2^{r_{2}}\left|\mu_{+}\right|} \int_{\mathbb{R}_{+}^{r} / \mathcal{O}_{+}^{\times}} N y^{s-1} \sum_{\eta>0} N \eta^{-1}\left|\lambda_{\pi}(\eta)\right|^{2}\left|\mathbf{K}_{k}(\eta \kappa y)\right|^{2} d y^{\times} \\
& =\left|a_{f}(1)\right|^{2} \frac{\sqrt{|D|}}{2^{r_{2}}} \sum_{(\eta)} N \eta^{-1}\left|\lambda_{\pi}(\eta)\right|^{2} \int_{\mathbb{R}_{+}^{r}} N y^{s-1}\left|\mathbf{K}_{k}(\eta \kappa y)\right|^{2} d y^{\times}
\end{aligned}
$$

(note that the factor of $\left|\mu_{+}\right|$vanished because $\mathcal{O}^{+} / \mathcal{O}_{+}^{\times}$counts each ideal with multiplicity $\left.\left|\mu_{+}\right|\right)$

$$
\begin{aligned}
& =\left|a_{f}(1)\right|^{2} \frac{\sqrt{|D|} N \kappa^{1-s}}{2^{r_{2}}} \sum_{(\eta)} \frac{\left|\lambda_{\pi}(\eta)\right|^{2}}{N \eta^{s}} \int_{\mathbb{R}_{+}^{r}} N y^{s-1}\left|\mathbf{K}_{k}(y)\right|^{2} d y^{\times} \\
& =\left|a_{f}(1)\right|^{2} \frac{|D|^{s-1 / 2}}{2^{r_{2}}} L\left(s, \operatorname{sym}^{2} \pi\right) \frac{\zeta_{F}(s)}{\zeta_{F}(2 s)} \int_{\mathbb{R}_{+}^{r}} N y^{s-1}\left|\mathbf{K}_{k}(y)\right|^{2} d y^{\times} .
\end{aligned}
$$

We only need the value of the integral at $s=1$, and to calculate it we expand it as a product over the infinite places. The factor at a real place is

$$
\int_{0}^{\infty} y^{k_{i}} \exp \left(-4 \pi k_{i} y\right) d y^{\times}=(4 \pi)^{-k_{i}} \Gamma\left(k_{i}\right) .
$$

At a complex place, it is

$$
\begin{aligned}
\int_{0}^{\infty} & y^{k_{i}+2} \sum_{j=0}^{k_{i}}\left(\begin{array}{c}
k_{i} \\
j
\end{array}\right) K_{k_{i} / 2-j}^{2}(4 \pi y) d y^{\times} \\
& =(4 \pi)^{-k_{i}-2} \sum_{j=0}^{k_{i}}\left(\begin{array}{c}
k_{i} \\
j
\end{array}\right) \int_{0}^{\infty} y^{k_{i}+2} K_{k_{i} / 2-j}^{2}(y) d y^{\times} \\
& =(4 \pi)^{-k_{i}-2} \frac{2^{k_{i}-1} \Gamma\left(1+k_{i} / 2\right)^{2}}{\Gamma\left(2+k_{i}\right)} \sum_{j=0}^{k_{i}}\left(\begin{array}{c}
k_{i} \\
j
\end{array}\right) \Gamma(1+j) \Gamma\left(1+k_{i}-j\right) \\
& =(4 \pi)^{-k_{i}-2} \frac{2^{k_{i}-1} \Gamma\left(1+k_{i} / 2\right)^{2}}{\Gamma\left(2+k_{i}\right)}\left(k_{i}+1\right) ! \\
& =2^{-5} \pi^{-2}(2 \pi)^{-k_{i}} \Gamma\left(1+k_{i} / 2\right)^{2} .
\end{aligned}
$$

Combining these, we have the following expression for $\operatorname{Res}_{s=1}\left\langle E(s, z) F_{k}, F_{k}\right\rangle$ :

$$
\begin{aligned}
\operatorname{Res}_{s=1}\left\langle E(s, z) F_{k}, F_{k}\right\rangle=\left|a_{f}(1)\right|^{2} & \frac{\sqrt{|D|}}{2^{6 r_{2}} \pi^{2 r_{2}}} L\left(1, \operatorname{sym}^{2} \pi\right) \frac{\operatorname{Res}_{s=1} \zeta_{F}(s)}{\zeta_{F}(2)} \\
& \times \prod_{i \leq r_{1}}(4 \pi)^{-k_{i}} \Gamma\left(k_{i}\right) \prod_{i>r_{1}}(2 \pi)^{-k_{i}} \Gamma\left(1+k_{i} / 2\right)^{2} .
\end{aligned}
$$

Dividing by $\operatorname{Res}_{s=1} \phi(s)$ we obtain the required relation between $\left|a_{f}(1)\right|^{2}$ and $\left\langle F_{k}, F_{k}\right\rangle$,

$$
\left\langle F_{k}, F_{k}\right\rangle=\left|a_{f}(1)\right|^{2} \frac{|D| L\left(1, \operatorname{sym}^{2} \pi\right)}{2^{7 r_{2}-1} \pi^{r_{1}+3 r_{2}}} \prod_{i \leq r_{1}}(4 \pi)^{-k_{i}} \Gamma\left(k_{i}\right) \prod_{i>r_{1}}(2 \pi)^{-k_{i}} \Gamma\left(1+k_{i} / 2\right)^{2} .
$$


11.3. Volume computations. In this section we compute the volume element in the cusp of $Y$ and use this with our computation of the residue of $E(s, z)$ to calculate the volume of $Y$. As in Efrat [5], we shall introduce simplified coordinates in the cusp, defined using the matrix $A$ from section 2.2. We define the coordinates $Y_{0}, \ldots, Y_{r-1}$ by

$$
\left(\begin{array}{c}
\log Y_{0} \\
Y_{1} \\
\vdots \\
Y_{r-1}
\end{array}\right)=\left(\begin{array}{cccc}
1 & 1 & \ldots & 2 \\
e_{1}^{1} & e_{2}^{1} & \ldots & e_{r}^{1} \\
\vdots & & & \\
e_{1}^{r-1} & e_{2}^{r-1} & \ldots & e_{r}^{r-1}
\end{array}\right)\left(\begin{array}{c}
\log y_{1} \\
\log y_{2} \\
\vdots \\
\log y_{r}
\end{array}\right)
$$

so that

$$
\left(\begin{array}{c}
\log y_{1} \\
\log y_{2} \\
\vdots \\
\log y_{r}
\end{array}\right)=\left(\begin{array}{cccc}
1 / n & \log \left|\epsilon_{1}^{1}\right| & \ldots & \log \left|\epsilon_{r-1}^{1}\right| \\
\vdots & & & \\
1 / n & \log \left|\epsilon_{1}^{r}\right| & \ldots & \log \left|\epsilon_{r-1}^{r}\right|
\end{array}\right)\left(\begin{array}{c}
\log Y_{0} \\
Y_{1} \\
\vdots \\
Y_{r-1}
\end{array}\right)
$$

We shall compute the volume form of $\mathbb{H}_{F}$ with respect to the new system of coordinates $Y_{0}, \ldots, Y_{r-1}, x_{1}, \ldots, x_{r}$. As we are not changing the $x$-coordinates at all we may omit them from our calculations, and only compute the form $\bigwedge_{i} d y_{i} / y_{i}$ with respect to $\left\{Y_{i}\right\}$. The Jacobian of the change of coordinates is

$$
\left(\begin{array}{c}
\partial y_{1} \\
\partial y_{2} \\
\vdots \\
\partial y_{r}
\end{array}\right)=\left(\begin{array}{cccc}
\frac{y_{1}}{n Y_{0}} & y_{1} \log \left|\epsilon_{1}^{1}\right| & \ldots & y_{1} \log \left|\epsilon_{r-1}^{1}\right| \\
\vdots & & & \\
\frac{y_{r}}{n Y_{0}} & y_{r} \log \left|\epsilon_{1}^{r}\right| & \ldots & y_{r} \log \left|\epsilon_{r-1}^{r}\right|
\end{array}\right)\left(\begin{array}{c}
\partial Y_{0} \\
\partial Y_{1} \\
\vdots \\
\partial Y_{r-1}
\end{array}\right) \text {, }
$$

and we need to calculate its determinant which is

$$
\frac{1}{n Y_{0}} \prod_{i=1}^{r} y_{i} \operatorname{det}\left(\begin{array}{cccc}
1 & \log \left|\epsilon_{1}^{1}\right| & \ldots & \log \left|\epsilon_{r-1}^{1}\right| \\
\vdots & & & \\
1 & \log \left|\epsilon_{1}^{r}\right| & \ldots & \log \left|\epsilon_{r-1}^{r}\right|
\end{array}\right) .
$$

We shall calculate this determinant by a minor expansion along the first column. The absolute value of the determinant of the $(1, i)$ th minor is the regulator $R^{+}$of $\mathcal{O}_{+}^{\times}$times $1 / 2$ for every complex place we are expanding over. The index of $\mathcal{O}_{+}^{\times}$in $\mathcal{O}^{\times}$is $2^{r_{1}-1+\delta_{r_{1} 0}}$ so $R^{+}=2^{r_{1}-1+\delta_{r_{1} 0}} R$, and the alternating sum of the minors is $\left(r_{1}+2 r_{2}\right) 2^{r_{1}-r_{2}-1+\delta_{r_{1} 0}} R=n 2^{r_{1}-r_{2}-1+\delta_{r_{1} 0}} R$. The expression for $d v$ in terms of our new coordinate system is therefore

$$
d v=\frac{2^{r_{1}-r_{2}-1+\delta_{r_{1} 0}} R}{Y_{0}^{2}} \bigwedge_{i=0}^{r-1} d Y_{i} \wedge d x .
$$

We now verify that the main term appearing in $I_{\phi}(T)$ during the contour shift in Lemma 12 and equation (49) is in fact $\langle E(z \mid g), 1\rangle / \operatorname{Vol}(Y)\left\langle\phi F_{k}, F_{k}\right\rangle$. The residue is equal to

$$
V_{c}^{-1} G(-1,0) \operatorname{Res}_{s=1} \phi(s)\left\langle\phi F_{k}, F_{k}\right\rangle,
$$

and it may easily be seen that $\langle E(z \mid g), 1\rangle=2^{-r_{2}}\left|\mu_{+}\right|^{-1} \sqrt{|D|} G(-1,0)$. Therefore to show that the two expressions are equal we only need to show that $\operatorname{Vol}(Y)=$ $V_{c} 2^{-r_{2}}\left|\mu_{+}\right|^{-1} \sqrt{|D|}\left(\operatorname{Res}_{s=1} \phi(s)\right)^{-1}$. This follows easily from the standard method 
of computing the volume of fundamental domains using Eisenstein series, and substituting the value of $\operatorname{Res}_{s=1} \phi(s)$ gives

$$
\operatorname{Vol}(Y)=\frac{2^{-4 r_{2}+1}|D|^{3 / 2} \zeta_{F}(2)}{\pi^{n}}
$$

\section{ACKNOWLEDGEMENTS}

The author would like to thank his adviser Peter Sarnak for suggesting this problem as part of his thesis and providing much guidance and encouragement in the course of his work, and the referees for a very thorough reading of the manuscript.

\section{REFERENCES}

[1] A. Borel, N. Wallach, Continuous Cohomology, Discrete Subgroups, and Representations of Reductive Groups, Mathematical Surveys and Monographs, no. 67, American Mathematical Society (2000). MR1721403 (2000j:22015)

[2] T. Berger, G. Harcos, l-adic representations associated to modular forms over imaginary quadratic fields, IMRN (2007), article ID rnm113. MR.2380006 (2008m:11109)

[3] D. Blasius, Hilbert modular forms and the Ramanujan conjecture. In Noncommutative Geometry and Number Theory, Aspects Math., E37, pages 35-56. Vieweg, Wiesbaden, 2006. MR2327298 (2008g:11077)

[4] V. Blomer, G. Harcos, Twisted L-functions over number fields and Hilbert's eleventh problem, Geom. Funct. Anal. 20 (2010), no. 1, 1-52. MR2647133

[5] I. Efrat, The Selberg Trace Formula for $\operatorname{PSL}(2, \mathbb{R})^{n}$, Memoirs of the American Mathematical Society, no. 359, American Mathematical Society (1987). MR874084 (88e:11041)

[6] P. D. T. A. Elliott, C. J. Moreno, F. Shahidi, On the absolute value of Ramanujan's $\tau$ function, Math. Ann. 266 (1984), 507-511. MR735531 (85f:11030)

[7] T. Finis, F. Grunewald, P. Tirao, The cohomology of lattices in $S L(2, \mathbb{C})$, Exp. Math. 19 (2010), no. 1, 29-63. MR2649984

[8] P. Garrett, Decomposition of Eisenstein series, Rankin triple products, Ann. of Math. (2) 125 (1987), 209-235. MR881269 (88m:11033)

[9] S. Gelbart, H. Jacquet, A relation between automorphic representations of $G L(2)$ and $G L(3)$, Ann. Sci. École Norm. Sup. 11 (1978), 471-552. MR533066 (81e:10025)

[10] D. Goldfeld, J. Hoffstein, D. Lieman, Appendix: An effective zero-free region, Ann. of Math. (2) 140 (1994), 177-181. MR:1289494 (95m:11048)

[11] I.S. Gradshteyn, I.M. Ryzhik, Table of Integrals, Series and Products, Academic Press (1980). MR1773820 (2001c:00002)

[12] M. Harris, D. Soudry, R. Taylor, l-adic representations associated to modular forms over imaginary quadratic fields, Invent. Math. 112 (1993), 377-411. MR.1213108 (94d:11035)

[13] J. Hoffstein, P. Lockhart, Coefficients of Maass forms and the Siegel zero, Ann. of Math. (2) 140 (1994), 161-181. MR1289494 (95m:11048)

[14] R. Holowinsky, A sieve method for shifted convolution sums, Duke Math. J. 146 (2009), no. 3, 401-448. MR2484279 (2010b:11127)

[15] R. Holowinsky, Sieving for mass equidistribution, Ann. of Math. (2) 172 (2010), no. 2, 14991516. MR 2680498

[16] R. Holowinsky, K. Soundararajan, Mass equidistribution for Hecke eigenforms, Ann. of Math. (2) $\mathbf{1 7 2}$ (2010), no. 2, 1517-1528. MR2680499

[17] A. Ichino, Trilinear forms and the central values of triple product L-functions, Duke Math. J. 145 vol. 2 (2008), 281-307. MR2449948 (2009i:11066)

[18] H. Iwaniec, E. Kowalski, Analytic Number Theory, American Mathematical Society Colloquium Publications, 53. American Mathematical Society, Providence, RI, 2004. xii + 615 pp. MR2061214 (2005h:11005)

[19] H. Jacquet, R. P. Langlands, Automorphic Forms on $G L_{2}$, vol. 2, Lecture Notes in Mathematics, Vol. 278, Springer-Verlag, Berlin, New York. MR 0401654 (53:5481)

[20] E. Lindenstrauss, Invariant measures and arithmetic quantum unique ergodicity, Ann. of Math. (2) 163 (2006), 705-741. MR2195133 (2007b:11072) 
[21] E. Lindenstrauss, On quantum unique ergodicity for $\Gamma \backslash \mathbb{H} \times \mathbb{H}$, IMRN (2001), no. 17, 913-933. MR:1859345 (2002k:11076)

[22] S.-C. Liu, Equidistribution of Hecke eigenforms on the Hilbert modular varieties, J. Number Theory 127 (2007), no. 1, 1-9. MR 2351660 (2009a:11109)

[23] W. Luo, P. Sarnak, Mass equidistribution for Hecke eigenforms, Comm. Pure Appl. Math. 56 (2003), no. 7, 874-891. MR1990480 (2004e:11038)

[24] S. Marshall, Quantum chaos and triple product identities on $S L(2, \mathbb{C})$, preprint, available as arXiv.org:math/:1006.3303v1.

[25] P. Michel, A. Venkatesh, The subconvexity problem for $G L_{2}$, Publ. Math. Inst. Hautes Études Sci. 111 (2010), no. 1, 171-271. MR2653249

[26] H. Montgomery, R. Vaughan, Multiplicative Number Theory. I. Classical Theory.Cambridge University Press, 2007. MR2378655 (2009b:11001)

[27] M. Nair, Multiplicative functions of polynomial values in short intervals, Acta Arith. 62 (1992), no. 3, 257-269. MR1197420 (94b:11093)

[28] M. Nair, G. Tenenbaum, Short sums of certain arithmetic functions, Acta Math. 180 (1998), no. 1, 119-144. MR1618321 (99j:11110)

[29] P. Nelson, Mass equidistribution of Hilbert modular eigenforms, preprint, available as arxiv.org:1011.1291v1.

[30] S. Nonnenmacher, A. Voros, Chaotic eigenfunctions in phase space, J. Stat. Phys. 92 (1998), no. 3-4, 431-518. MR.1649013 (2000c:81079)

[31] Z. Rudnick, On the asymptotic distribution of zeros of modular forms, IMRN 34 (2005), 2059-2074. MR.2181743 (2006k:11099)

[32] Z. Rudnick, P. Sarnak, The behaviour of eigenstates of arithmetic hyperbolic manifolds, Comm. Math. Phys. 161 (1994), 195-213. MR.1266075 (95m:11052)

[33] P. Sarnak, The arithmetic and geometry of some hyperbolic three manifolds, Acta Math. 151 (1983), 253-295. MR723012 (85d:11061)

[34] P. Sarnak, Estimates for Rankin-Selberg L-functions and quantum unique ergodicity, J. Funct. Anal. 184 (2001), 419-453. MR.1851004 (2003c:11050)

[35] B. Shiffman, S. Zelditch, Distribution of zeros of random and quantum chaotic sections of positive line bundles, Comm. Math. Phys. 200 (1999), no. 3, 661-683. MR.1675133 (2001j:32018)

[36] H. Shimizu, On discontinuous groups acting on the product of the upper half planes, Ann. of Math. (2) 77 (1963), 33-71. MR.0145106 (26:2641)

[37] L. Silberman, A. Venkatesh, Quantum unique ergodicity on locally symmetric spaces I, Geom. Funct. Anal. 17 (2007), no. 3, 960-998. MR2346281(2009a:81072)

[38] A. I. Šnirel'man, Ergodic properties of eigenfunctions, Uspekhi Mat. Nauk 29 (1974), no. 6 (180), 181-182. MR0402834 (53:6648)

[39] K. Soundararajan, Weak subconvexity for central values of L-functions, Ann. of Math. (2) 172 (2010), no. 2, 1469-1498. MR 2680497

[40] K. Soundararajan, Quantum unique ergodicity for $S L_{2}(\mathbb{Z}) \backslash \mathbb{H}$, Ann. of Math. (2) 172 (2010), no. 2, 1529-1538. MR2680500

[41] T. Watson, Rankin triple products and quantum chaos, Ph.D. Thesis, Princeton University (eprint available at http://www.math.princeton.edu/ tcwatson/ watson_thesis_final.pdf) (2001) MR.2703041

[42] S. Zelditch, Pseudodifferential analysis on hyperbolic surfaces, J. Funct. Anal. 68 (1986), no. 1, 72-105. MR850155 (87j:58092)

[43] S. Zelditch, The averaging method and ergodic theory for pseudo-differential operators, J. Funct. Anal. 82 (1989), no. 1, 38-68. MR976312 (91e:58194)

School of Mathematics, The Institute for Advanced Study, Einstein Drive, PrinceTON, NEW JERSEY 08540

E-mail address: slm@math.princeton.edu 